\title{
KOBAYASHI HYPERBOLICITY OF THE COMPLEMENTS OF GENERAL HYPERSURFACES OF HIGH DEGREE
}

\author{
DAMIAN BROTBEK AND YA DENG
}

\begin{abstract}
In this paper, we prove that in any projective manifold, the complements of general hypersurfaces of sufficiently large degree are Kobayashi hyperbolic. We also provide an effective lower bound on the degree. This confirms a conjecture by S. Kobayashi in 1970. Our proof, based on the theory of jet differentials, is obtained by reducing the problem to the construction of a particular example with strong hyperbolicity properties. This approach relies the construction of higher order logarithmic connections allowing us to construct logarithmic Wronskians. These logarithmic Wronskians are the building blocks of the more general logarithmic jet differentials we are able to construct.

As a byproduct of our proof, we prove a more general result on the orbifold hyperbolicity for generic geometric orbifolds in the sense of Campana, with only one component and large multiplicities. We also establish a Second Main Theorem type result for holomorphic entire curves intersecting general hypersurfaces, and we prove the Kobayashi hyperbolicity of the cyclic cover of a general hypersurface, again with an explicit lower bound on the degree of all these hypersurfaces.
\end{abstract}

\section{INTRODUCTION}

A complex space $X$ is said to be Kobayashi hyperbolic if the (intrinsically defined) Kobayashi pseudo distance $d_{X}$ is a distance, meaning that $d_{X}(p, q)>0$ for $p \neq q$ in $X$. One can easily see that a Kobayashi hyperbolic complex space $X$ does not contain any non-constant entire holomorphic curve $f: \mathbb{C} \rightarrow X$ (this last property is called Brody hyperbolicity). When $X$ is compact, by a well-known theorem of Brody [Bro78], these two definitions of hyperbolicity are equivalent. However, in general, we have many examples of complex manifolds which are Brody hyperbolic but not hyperbolic in the sense of Kobayashi, see for instance [Kob98].

In 1970, Kobayashi made the following conjecture [Kob70], which is often called the logarithmic Kobayashi conjecture in the literatures.

Conjecture 0.1 (Kobayashi). The complement $\mathbb{P}^{n} \backslash D$ of a general hypersurface $D \subset \mathbb{P}^{n}$ of sufficiently large degree $d \geqslant d_{n}$ is Kobayashi hyperbolic.

As is well known, Conjecture 0.1 is simpler to approach when $D$ is replaced by a simple normal crossing divisor with several components. When $D=\sum_{i=1}^{2 n+1} H_{i}$ with $\left\{H_{i}\right\}_{i=1, \ldots, 2 n+1}$ hyperplanes of $\mathbb{P}^{n}$ in general position, it was proved by Fujimoto [Fuj72] and Green [Gre77] that $\mathbb{P}^{n} \backslash D$ is Kobayashi hyperbolic. More generally, Noguchi-Winkelmann-Yamanoi [NWY07, NWY08, NWY13] and Lu-Winkelmann [LW12] even proved a stronger result towards the logarithmic Green-Griffiths conjecture: if $(Y, D)$ is a pair of log general type with logarithmic irregularity $h^{0}\left(Y, \Omega_{Y}(\log D)\right) \geqslant \operatorname{dim} Y$, then $(Y, D)$ is weakly hyperbolic. Here we say a $\log$ pair $(Y, D)$ is weakly hyperbolic if all entire curves in $Y \backslash D$ lie in a proper subvariety $Z \subsetneq Y$. When the logarithmic irregularity is strictly smaller than the dimension of the manifold, or equivalently the number of irreducible components of $D$ are less or equal than the dimension of the manifold, much less is known for the general logarithmic Green-Griffiths conjecture. In [Rou03, Rou09]

Date: Monday $1^{\text {st }}$ April, 2019.

2010 Mathematics Subject Classification. 32Q45, 30D35, 14 E99.

Key words and phrases. Kobayashi hyperbolicity, orbifold hyperbolicity, logarithmic-orbifold Kobayashi conjecture, Second Main Theorem, jet differentials, logarithmic Demailly tower, higher order log connections, logarithmic Wronskians. 
Rousseau dealt with the Kobayashi hyperbolicity of $\mathbb{P}^{2} \backslash D$ where $D$ consists of two irreducible curves of certain degrees. More recently, in [BD17] we proved a more general result concerning the hyperbolicity of the complement of a sufficiently ample divisor with several components.

Theorem 0.2 ([BD17]). Let $Y$ be a smooth projective variety of dimension $n$ and let $c \geqslant n$. Let $L$ be a very ample line bundle on $Y$. For any $m \geqslant(4 n)^{n+2}$ and for general hypersurfaces $H_{1}, \ldots, H_{c} \in\left|L^{m}\right|$, writing $D=\sum_{i=1}^{c} H_{i}$, the logarithmic cotangent bundle $\Omega_{Y}(\log D)$ is almost ample. In particular, $Y \backslash D$ is Kobayashi hyperbolic and hyperbolically embedded into $Y$.

This result can be seen as a logarithmic analogue of a conjecture of Debarre, which was established by the first author and Darondeau in [BD18] and independently by Xie [Xie18].

Let us now focus on the case of one component as in Conjecture 0.1. In the case $n=2$, the first proof to Conjecture 0.1 was provided by Siu-Yeung [SY96], with a very high degree bound, which was later improved to $d \geqslant 15$ by El Goul [EG03] and $d \geqslant 14$ by Rousseau [Rou09]. Building on ideas of Voisin [Voi96, Voi98], Siu [Siu04], Diverio-Merker-Rousseau [DMR10], the first step towards the general case in Conjecture 0.1 was made by Darondeau in [Dar16b], in which he proved the weak hyperbolicity of $\mathbb{P}^{n} \backslash D$ for general hypersurfaces $D$ of degree $d \geqslant(5 n)^{2} n^{n}$. Very recently, based on his strategy outlined in [Siu04], in [Siu15] Siu made an important progress towards Conjecture 0.1, in which he showed that $\mathbb{P}^{n} \backslash D$ is Brody hyperbolic for $D$ a general hypersurface of degree $d \geqslant d_{n}^{*}$, where $d_{n}^{*}$ is some (non-explicit) function depending on $n$.

The goal of the present paper is to prove Conjecture 0.1 with an effective estimate on the lower degree bound $d_{n}$. We also prove a Second Main Theorem type result, orbifold hyperbolicity of a general orbifold with one component and high multiplicity, and Kobayashi hyperbolicity of the cyclic cover of a general hypersurface of large degree.

Main Theorem (=Corollaries 4.6, 4.9 and 4.11). Let $Y$ be a smooth projective variety of dimension $n \geqslant 2$. Fix any very ample line bundle $A$ on $Y$. Then for a general smooth hypersurface $D \in\left|A^{d}\right|$ with

$$
d \geqslant(n+2)^{n+3}(n+1)^{n+3} \sim_{n \rightarrow \infty} e^{3} n^{2 n+6},
$$

(i) The complement $Y \backslash D$ is hyperbolically embedded into $Y$. In particular, $Y \backslash D$ is Kobayashi hyperbolic.

(ii) For any holomorphic entire curve (possibly algebraically degenerate) $f: \mathbb{C} \rightarrow Y$ which is not contained in D, one has

$$
T_{f}(r, A) \leqslant N_{f}^{(1)}(r, D)+C\left(\log T_{f}(r, A)+\log r\right) \quad \| .
$$

Here $T_{f}(r, A)$ is the Nevanlinna order function, $N_{f}^{(1)}(r, D)$ is the truncated counting function, and the symbol $\|$ means that the inequality holds outside a Borel subset of $(1,+\infty)$ of finite Lebesgue measure.

(iii) The (Campana) orbifold $\left(Y,\left(1-\frac{1}{d}\right) D\right)$ is orbifold hyperbolic, i.e. there exists no entire curve $f: \mathbb{C} \rightarrow Y$ so that

$$
f(\mathbb{C}) \not \subset D \quad \text { with } \operatorname{mult}_{t}\left(f^{*} D\right) \geqslant d \quad \forall t \in f^{-1}(D) .
$$

(iv) Let $\pi: X \rightarrow Y$ be the cyclic cover of $Y$ obtained by taking the $d$-th root along $D$. Then $X$ is Kobayashi hyperbolic.

To the best of our knowledge, Main Theorem (iii) is the first general result on the orbifold Kobayashi conjecture [Rou10, Conjecture 5.5] dealing with general orbifolds with only one component. We note that Main Theorem (i) immediately follows from Main Theorem (iii) in view of the definition of orbifold hyperbolicity and our previous results [Bro17, Den17]. We also observe that Main Theorem (iii) implies Main Theorem (iv) since $\pi:(X, \varnothing) \rightarrow\left(Y,\left(1-\frac{1}{d}\right) D\right)$ is a $d$-folded unramified cover in the category of orbifold. The only result of the type of Main Theorem (iv) we are aware of is due to Roulleau-Rousseau [RR13], who proved that for a very 
general hypersurface $D$ in $\mathbb{P}^{n}$ of degree $d \geqslant 2 n+2$, the cyclic cover $X$ of $Y$ obtained by taking the $d$-th root along $D$ is algebraically hyperbolic.

Let us mention that in a recent preprint [RY18], which appeared after the first version of the present paper was made publicly available, Riedl-Yang provide a short proof of Conjecture 0.1 with an effective bound on $d_{n}$ (which is slightly worse than the bound we give here). However, their proof relies heavily on a series of work by Darondeau [Dar16a, Dar16b, Dar16c] whereas our proof is essentially self-contained.

Our approach is inspired by our previous works [Bro17, Den17, BD17]. Those works were motivated by the compact counterpart of Kobayashi conjecture, also conjectured in [Kob70] by Kobayashi: a general hypersurface $X \subset \mathbb{P}^{n}$ of sufficiently high degree $d \geqslant d_{n}^{\prime}$ is Kobayashi hyperbolic. There are now several proofs of this result, [Siu15,Bro17] and more recently [Dem18]. Here we will provide a logarithmic counterpart to the approach of [Bro17] as well as the work [Den17].

Let us now outline the main points of the proof of our main result. First we observe that the first statement of our main result will follow from the Brody hyperbolicity of $Y \backslash D$ in view of a theorem of Green [Gre77] and the results established in [Bro17, Den17]. In order to control the entire curves in $Y \backslash D$ we rely on the theory of logarithmic jet differentials. Logarithmic jet differentials on the pair $(Y, D)$ are higher order generalizations of symmetric differential forms with logarithmic poles along $D$ and provide obstructions to the existence of entire curves. Roughly speaking, in order to prove that $Y \backslash D$ is Brody hyperbolic it suffices to construct many logarithmic jet differential forms on $(Y, D)$ vanishing along some ample divisor and control their geometry. Let us also observe that in general it is critical to use higher order jet differentials and not merely logarithmic symmetric differential forms. In general one has to go at least to order $k=\operatorname{dim} Y$ (see e.g. [Div09, Theorem 8]).

Let us now explain the approach we use to construct logarithmic jet differential forms. For simplicity, we suppose until the end of this section that $Y=\mathbb{P}^{n}$ and that $A=\mathscr{O}_{\mathbb{P}^{n}}(1)$. The first step is to introduce higher order logarithmic connections. More precisely for any integer $d \geqslant 1$, any smooth $D \in\left|\mathscr{O}_{\mathbb{P}^{n}}(d)\right|$ and any $k \geqslant 0$ we define the $k$-th order logarithmic connection associated to the pair $\left(\mathbb{P}^{n}, D\right)$

$$
\nabla_{D}^{k}: \mathscr{O}_{\mathbb{P}^{n}}(d) \rightarrow E_{k, k}^{\mathrm{GG}} \Omega_{\mathbb{P}^{n}}(\log D) \otimes \mathscr{O}_{\mathbb{P}^{n}}(d)
$$

by setting

$$
\nabla_{D}^{k} s=\sigma d^{k}\left(\frac{s}{\sigma}\right)
$$

where $D=(\sigma=0)$ and $s \in \Gamma\left(U, \mathscr{O}_{\mathbb{P}^{n}}(d)\right)$ for some open set $U \subset \mathbb{P}^{n}$. Here $E_{k, k}^{\mathrm{GG}} \Omega_{\mathbb{P}^{n}}(\log D)$ denotes the vector bundle of logarithmic jet differentials of order $k$ and weighted degree $k$. See $\S 1.2$ and $\S 2.2$ for more details. The crucial point is the following tautological equality for any $k \geqslant 1$

$$
\nabla_{D}^{k} \sigma=0
$$

Next, we follow the general strategy in [BD18, Bro17, BD17] which consists in reducing the general case to the construction of a particular example $\left(\mathbb{P}^{n}, D\right)$ satisfying a certain ampleness property, which implies Brody hyperbolicity and which is a Zariski open property. Such examples are indeed suitable deformations of Fermat type hypersurfaces. For some suitably chosen parameters $\delta, r, \varepsilon, k \in \mathbb{N}^{*}$, consider the hypersurface $D_{\mathbf{a}} \subset \mathbb{P}^{n}$ defined by a polynomial of degree 
$d:=\varepsilon+(r+k) \delta$ in the form

$$
F(\mathbf{a})=\sum_{\substack{I=\left(i_{0}, \ldots, i_{n}\right) \\ i_{0}+\cdots+i_{n}=\delta}} a_{I} z^{(r+k) I},
$$

where we use the multi-index notation $z^{(r+1) I}=\left(z_{0}^{i_{0}} \cdots z_{n}^{i_{n}}\right)^{r+k}$ for $I=\left(i_{0}, \ldots, i_{n}\right)$ and homogeneous coordinates $\left[z_{0}, \ldots, z_{n}\right]$ on $\mathbb{P}^{n}$, and the $a_{I}$ 's are homogeneous polynomials of degree $\varepsilon \geqslant 1$ in $\mathbb{C}\left[z_{0}, \ldots, z_{n}\right]$. Write $D_{\mathbf{a}}=(F(\mathbf{a})=0) \subset \mathbb{P}^{n}$. By considering the tautological relation $(0.2)$ for any $j=1, \ldots, k$ we obtain the following equalities

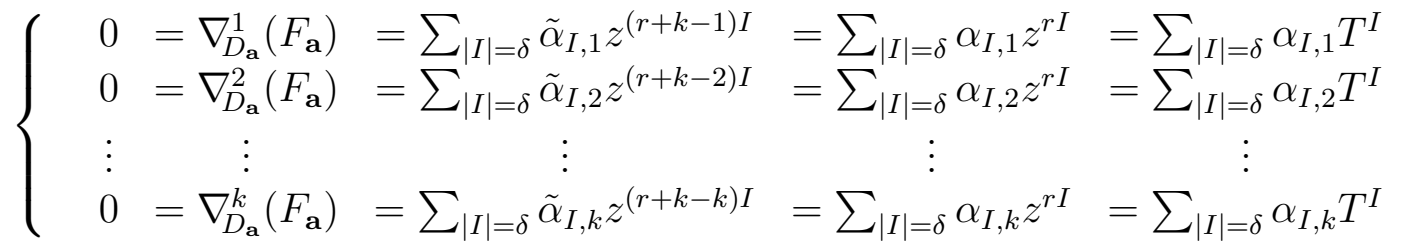

Here $\left(T_{0}, \ldots, T_{n}\right):=\left(z_{0}^{r}, \ldots, z_{n}^{r}\right)$, and for each $I$ and $i$, one has

$$
\alpha_{I, i} \in H^{0}\left(\mathbb{P}^{n}, E_{i, i}^{\mathrm{GG}} \Omega_{\mathbb{P}^{n}}\left(\log D_{\mathbf{a}}\right) \otimes \mathscr{O}_{\mathbb{P}^{n}}(\varepsilon+k \delta)\right) .
$$

One should think of these elements as some holomorphic functions on some suitable logarithmic jet space $\mathbb{P}_{k}^{n}\left(D_{\mathbf{a}}\right)$ (the logarithmic version of the Demailly-Semple jet tower constructed in [DL01]). Once suitably interpreted, (0.4) allows us to construct a rational map

$$
\begin{aligned}
\Phi_{\mathbf{a}}: \mathbb{P}_{k}^{n}\left(D_{\mathbf{a}}\right) & -\rightarrow \mathscr{Y} \\
w & \stackrel{\operatorname{loc}}{\mapsto}\left(\operatorname{Span}\left(\alpha_{\bullet, 1}(w), \alpha_{\bullet, 2}(w), \ldots, \alpha_{\bullet, k}(w)\right) ;\left[z_{0}^{r}, z_{1}^{r}, \ldots, z_{n}^{r}\right]\right)
\end{aligned}
$$

where $\alpha_{\bullet}, i(w):=\left(\alpha_{I, i}(w)\right)_{|I|=\delta} \in H^{0}\left(\mathbb{P}^{n}, \mathscr{O}_{\mathbb{P}^{n}}(\delta)\right)$, and $\mathscr{Y}$ is the universal complete intersections of codimension $k$ and multidegree $(\delta, \ldots, \delta)$ :

$$
\mathscr{Y}:=\left\{(\Delta,[T]) \in \operatorname{Gr}_{k}\left(H^{0}\left(\mathbb{P}^{n}, \mathscr{O}_{\mathbb{P}^{n}}(\delta)\right)\right) \times \mathbb{P}^{n} \mid \forall P \in \Delta, P([T])=0\right\} .
$$

If one denotes by $\mathscr{L}$ the Plücker line bundle on the $\operatorname{Grassmannian} \operatorname{Gr}_{k}\left(H^{0}\left(\mathbb{P}^{n}, \mathscr{O}_{\mathbb{P}^{n}}(\delta)\right)\right)$, by (0.5), for any $m \in \mathbb{N}^{*}$ and for $r$ large enough, the pull-back of every section in

$$
H^{0}\left(\mathscr{Y},\left.\mathscr{L}^{m} \otimes \mathscr{O}_{\mathbb{P}^{n}}(-1)\right|_{\mathscr{Y}}\right)
$$

induces a logarithmic jet differential equation on the pair $\left(\mathbb{P}^{n}, D_{\mathbf{a}}\right)$ vanishing along some ample divisor. Observe that when $k \geqslant n$, the projection map $\mathscr{Y} \rightarrow \operatorname{Gr}_{k}\left(H^{0}\left(\mathbb{P}^{n}, \mathscr{O}_{\mathbb{P}^{n}}(\delta)\right)\right)$ is generically finite, and thus the pull back of $\mathscr{L}$ to $\mathscr{Y}$ is a big and nef line bundle. Therefore, when $m$ is large enough, there are many global sections of $\left.\mathscr{L}^{m} \otimes \mathscr{O}_{\mathbb{P}^{n}}(-1)\right|_{\mathscr{Y}}$. Moreover, in view of a result of Nakamaye [Nak00] the base locus $\operatorname{Bs}\left(\left.\mathscr{L}^{m} \otimes \mathscr{O}_{\mathbb{P}^{n}}(-1)\right|_{\mathscr{Y}}\right)$ can be understood geometrically. Altogether this will allow us to control the geometry of the logarithmic jet differential forms we construct this way and eventually prove that for a general $\mathbf{a}$ and suitable restrictions on the different parameters, the pair $\left(\mathbb{P}^{n}, D_{\mathbf{a}}\right)$ satisfies a property which is Zariski open and implies, among other things, Brody hyperbolicity.

Let us however emphasize that there are many technical difficulties along the way. First of all, we are not able to work directly with the pair $\left(\mathbb{P}^{n}, D_{\mathbf{a}}\right)$, but with a pair $\left(H_{\mathbf{a}}, D_{\mathbf{a}}\right)$ which is biholomorphic to $\left(\mathbb{P}^{n}, D_{\mathbf{a}}\right)$ such that the family of all such pairs is easier to study. Secondly, the above rational map $\Phi_{\mathbf{a}}$ is not a regular morphism in general. Therefore the above strategy doesn't provide any information on what happens along the indeterminacy locus of this map but in order to obtain the strong hyperbolicity property we seek, we need some information on the entire logarithmic jet tower and not merely an open subset of it. Therefore as in [Bro17], we introduce a suitable modification of the logarithmic jet tower obtained by blowing up a suitable ideal sheaf induced by the logarithmic Wronskian construction we introduce here. The main difficulty lies in the description of elements $\alpha_{I, i}$ constructed above as holomorphic 
functions on the logarithmic Demailly jet tower. This forces us to introduce another version, more technically involved but more precise, of the logarithmic connections and the logarithmic Wronskians mentioned previously.

The paper is organized as follows. In $\S 1$, we recall the technical tools in studying the hyperbolicity of algebraic varieties, especially the logarithmic Demailly jet tower and the invariant logarithmic jet differentials. $\S 2$ is the main technical part of our paper. In this section, we develop our main tools in this paper: the higher order logarithmic connections and logarithmic Wronskians associated to families of global sections of a line bundle. We show that logarithmic Wronskians can be seen as a morphism from the jet bundle of a line bundle to the logarithmic invariant jet bundle. Based on this interpretation, we prove that for the ideal sheaf induced by the base ideal of logarithmic Wronskians, its cosupport lies on the set of singular jets in the $\log$ Demailly tower, and its blow-up is functorial under restrictions and families. This gives rise to a good compactification of the set of regular jets in the Demailly-Semple jet tower for the interior of the log pair. Using this construction we build a Zariski open property for the Brody hyperbolicity of the family of log pairs, and reduce our proof of the main theorem to find some particular examples. $\S 3$ is devoted to the construction of the family of these particular hypersurfaces in (0.3). In $\S 4$, we provide detailed proofs of the main theorem. We first prove (0.4) and (0.5), and show the existence of $D_{\mathbf{a}} \subset \mathbb{P}^{n}$ satisfying the above Zariski open property when we adjust the parameters. To prove Main Theorems (ii) to (iv), we reduce the problems to the existence of sufficiently many logarithmic jet differentials with a sufficiently negative twist.

\section{JET SPACES, JET DIFFERENTIALS AND JETS OF SECTIONS}

\subsection{Jet spaces and jet differentials.}

1.1.1. Jet spaces. Let $X$ be a complex manifold of dimension $n$. For any $k \in \mathbb{N}^{*}$, one defines $J_{k} X \rightarrow X$ to be the bundle of $k$-jets of germs of parametrized curves in $X$, that is, the set of equivalence classes of holomorphic maps $f:(\mathbb{C}, 0) \rightarrow X$, with the equivalence relation $f \sim_{k} g$ if and only if all derivatives $f^{(j)}(0)=g^{(j)}(0)$ coincide for $0 \leqslant j \leqslant k$, when computed in some (equivalently, any) local coordinate system of $X$ near $x$. Given any $f:(\mathbb{C}, 0) \rightarrow X$, we denote by $j_{k} f \in J_{k} X$ the class of $f$ in $J_{k} X$. There is a projection map $p_{k}: J_{k} X \rightarrow X$ defined by $p_{k}\left(j_{k} f\right)=f(0)$. Under this map, $J_{k} X$ is a $\mathbb{C}^{n k}$-fiber bundle over $X$. This can be seen as follows.

Let $U \subset X$ be an open subset. For any holomorphic 1-form $\omega \in \Gamma\left(U, \Omega_{U}\right)$ and $f:(\mathbb{C}, 0) \rightarrow U$, we set $f^{*} \omega:=A(t) d t$ and define the following functional:

$$
\begin{aligned}
d^{k-1} \omega: p_{k}^{-1}(U) & \rightarrow \mathbb{C} \\
j_{k} f & \mapsto A^{(k-1)}(0)
\end{aligned}
$$

One immediately checks that this is well defined. In the particular case $\omega=d \varphi$ for some $\varphi \in \mathscr{O}(U)$, one writes $d^{k} \varphi:=d^{k-1} \omega$. This construction allows us to see $J_{k} X$ as a $\mathbb{C}^{n k}$-fiber bundle over $X$. Indeed, given $\omega_{1}, \ldots, \omega_{n} \in \Gamma\left(U, \Omega_{U}\right)$ generating $\Omega_{X}$ at any point $x \in U$, then $\left\{d^{\ell} \omega_{i}\right\}_{0 \leqslant \ell \leqslant k-1,1 \leqslant i \leqslant n}$ gives rise to the local trivialization of $p_{k}^{-1}(U)$ :

$$
\begin{aligned}
p_{k}^{-1}(U) & \rightarrow U \times \mathbb{C}^{n k} \\
j_{k} f & \mapsto\left(f(0) ; d^{\ell} \omega_{i}\left(j_{\ell} f\right)\right)_{0 \leqslant \ell \leqslant k-1,1 \leqslant i \leqslant n}
\end{aligned}
$$

In this case the projection to the second factor $\mathbb{C}^{n k}$ is called the jet projection, and the natural coordinates of $\mathbb{C}^{n k}$ are called jet coordinates.

In particular, if $\left(z_{1}, \ldots, z_{n}\right)$ are local holomorphic coordinates on $U$ centered at a point $x \in U$, then $d z_{1}, \ldots, d z_{k}$ generates $\Omega_{U}$ at each point of $U$. Any germ of curve $f:(\mathbb{C}, 0) \rightarrow(X, x)$ can be written as

$$
f=\left(f_{1}, \ldots, f_{n}\right):(\mathbb{C}, 0) \rightarrow\left(\mathbb{C}^{n}, 0\right) .
$$


It follows from the trivialization (1.1.2) given by $\left\{d^{\ell} z_{j}\right\}_{1 \leqslant \ell \leqslant k, 1 \leqslant j \leqslant n}$ that the fiber $p_{k}^{-1}(x)$ can be identified with the set of $k$-tuples of vectors

$$
\left(\xi_{1}, \ldots, \xi_{k}\right)=\left(f^{\prime}(0), f^{\prime \prime}(0), \ldots, f^{(k)}(0)\right) \in \mathbb{C}^{n k} .
$$

Observe also that there is a natural $\mathbb{C}^{*}$-action on fibers of $J_{k} X$ defined by

$$
\lambda \cdot j_{k} f:=j_{k}(t \mapsto f(\lambda t)), \quad \forall \lambda \in \mathbb{C}^{*}, j_{k} f \in J_{k} X .
$$

With respect to the above trivialization, this action is described in jet coordinates by

$$
\lambda \cdot\left(f^{\prime}(0), f^{\prime \prime}(0), \ldots, f^{(k)}(0)\right)=\left(\lambda f^{\prime}(0), \lambda^{2} f^{\prime \prime}(0), \ldots, \lambda^{k} f^{(k)}(0)\right) .
$$

1.1.2. Jet differentials. Let us now recall the fundamental concept of jet differentials. For $X$ as above, any open subset $U \subset X$ and any integer $k \geqslant 1$, a jet differential of order $k$ on $U$ is an element $P \in \mathscr{O}\left(p_{k}^{-1}(U)\right)$. The (non-coherent) sheaf of jet differentials is defined to be $\mathscr{E}_{k, \bullet} \Omega_{X}:=\left(p_{k}\right)_{*} \mathscr{O}_{J_{k} X}$

The $\mathbb{C}^{*}$-action can be used to define the notion of of weight for jet differentials: a $k$-jet differential $P \in \mathscr{E}_{k, \bullet} \mathrm{GG} \Omega_{X}(U)=\mathscr{O}\left(p_{k}^{-1}(U)\right)$ is said to be of weight $m$ if for any $j_{k} f \in p_{k}^{-1}(U)$, one has

$$
P\left(\lambda \cdot j_{k} f\right)=\lambda^{m} P\left(j_{k} f\right)
$$

We thus define the Green-Griffiths sheaf $\mathscr{E}_{k, m} \mathrm{GG} \Omega_{X}$ of jet differentials of order $k$ and weighted degree $m$ to be the subsheaf of $\mathscr{E}_{k, \bullet}^{\mathrm{GG}} \Omega_{X}$, of jet differentials of weight $m$ with respect to the $\mathbb{C}^{*}$-action. With the above local coordinates, any element $P \in \mathscr{E}_{k, m} \mathrm{GG} \Omega_{X}(U)$ can be written as

$$
P\left(z, d z, \ldots, d^{k} z\right)=\sum_{|\alpha|=m} c_{\alpha}(z)\left(d^{1} z\right)^{\alpha_{1}}\left(d^{2} z\right)^{\alpha_{2}} \cdots\left(d^{k} z\right)^{\alpha_{k}}
$$

where $c_{\alpha}(z) \in \mathscr{O}(U)$ for any $\alpha:=\left(\alpha_{1}, \ldots, \alpha_{k}\right) \in\left(\mathbb{N}^{n}\right)^{k}$ and where we used the usual multi-index notation with the weighted degree $|\alpha|:=\left|\alpha_{1}\right|+2\left|\alpha_{2}\right|+\cdots+k\left|\alpha_{k}\right|$. From this it follows at once that $\mathscr{E}_{k, m} \mathrm{GG} \Omega_{X}$ is locally free, and we shall denote the associated vector bundle by $E_{k, m}^{\mathrm{GG}} \Omega_{X}$. One also defines $E_{k, \bullet}^{\mathrm{GG}} \Omega_{X}=\bigoplus_{m \geqslant 0} E_{k, m}^{\mathrm{GG}} \Omega_{X}$, which is in a natural way a bundle of graded algebras (the product is obtained simply by taking the product of polynomials).

Besides the multiplication, one can define for every $k, m \geqslant 0$, a $\mathbb{C}$-linear operator $d$ : $\mathscr{E}_{k, m} \Omega_{X} \rightarrow \mathscr{E}_{k+1, m+1} \Omega_{X}$ by

$$
(d P)\left(j_{k+1} f\right):=\frac{d}{d t}\left(P\left(j_{k} f(t)\right)\right)(0) .
$$

The fact that $d P$ is well defined and holomorphic follows from a local computation. This operator is coherent with the definition of $d^{k}$ above in the sense that for any holomorphic one form $\omega \in \Gamma\left(U, \Omega_{U}\right)$ on some open subset $U \subset X$, and any $k \in \mathbb{N}^{*}$ one has $d^{k} \omega=d\left(d^{k-1} \omega\right)$. For instance, this implies that $d^{k} \omega \in \mathscr{E}_{k+1, k+1} \Omega_{X}(U)$. In coordinates the operator $d$ can be computed as follows. Take an open subset $U \subset X$ with a coordinate chart $\left(z_{1}, \ldots, z_{n}\right)$, and let $P \in \mathscr{E}_{k, m} \mathrm{GG} \Omega_{X}(U)$ represented in coordinates by the expression (1.1.3), then $d P$ is given by

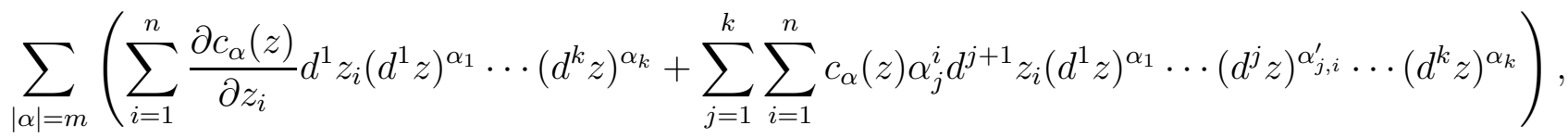
where $\alpha_{j, i}^{\prime}=\left(\alpha_{j}^{1}, \ldots, \alpha_{j}^{i-1}, \alpha_{j}^{i}-1, \alpha_{j}^{i+1}, \ldots, \alpha_{j}^{n}\right)$.

1.2. Logarithmic jet spaces and bundles. Let $X$ be a complex manifold (not necessarily compact), and let $D=\sum_{i=1}^{c} D_{i}$ be a simple normal crossing divisor on $X$, that is, all the components $D_{i}$ are smooth irreducible divisors that meet transversally. Such a pair $(X, D)$ is called a (smooth) $\log$ manifold. One denotes by $T_{X}(-\log D)$ the logarithmic tangent bundle of $X$ along $D$. By definition, it is the subsheaf of the holomorphic tangent bundle $T_{X}$ consisting of vector fields tangent to $D$. One can then show that under our assumptions on $D, T_{X}(-\log D)$ is a locally free sheaf. Let $U \subset X$ be an open subset of with local coordinates $\left(z_{1}, \ldots, z_{n}\right)$ such 
that for some $0 \leqslant c^{\prime} \leqslant c, D \cap U=\left(z_{1} \cdots z_{c^{\prime}}=0\right)$ and (up to reordering the components) one has $D_{i} \cap U=\left(z_{i}=0\right)$ for all $i=1 \ldots c^{\prime}$. Then $T_{X}(-\log D)$ is generated by

$$
z_{1} \frac{\partial}{\partial z_{1}}, \ldots, z_{c^{\prime}} \frac{\partial}{\partial z_{c^{\prime}}}, \frac{\partial}{\partial z_{c^{\prime}+1}}, \ldots, \frac{\partial}{\partial z_{n}} .
$$

Consider the dual of $T_{X}(-\log D)$, which is the locally free sheaf generated by

$$
\frac{d z_{1}}{z_{1}}, \ldots, \frac{d z_{c^{\prime}}}{z_{c^{\prime}}}, d z_{c^{\prime}+1}, \ldots, d z_{n}
$$

and denoted by $\Omega_{X}(\log D)$. The vector bundle $\Omega_{X}(\log D)$ is called the logarithmic cotangent bundle of $(X, D)$. We denote by $\mathscr{J}_{k}(X)$ the set of local holomorphic sections $\alpha: U \rightarrow J_{k} X$ of the $k$-jet bundle $J_{k} X \rightarrow X$, and $\mathscr{J}_{k}(X, \log D)$ the sheaf of germs of local holomorphic sections $\alpha$ of $J_{k} X$ such that for any $\omega \in \Omega_{X}(\log D)_{x},\left(d^{j-1} \omega\right)(\alpha)$ are all holomorphic for any $j=1, \ldots, k . \mathscr{J}_{k}(X, \log D)$ is called the logarithmic $k$-jet sheaf and $\alpha$ is called a logarithmic $k$-jet field. Here we observe that for any meromorphic 1 -form $\omega \in \mathscr{M}\left(U, \Omega_{X}\right)$, one can also define $d^{i} \omega$ for any $i=1, \ldots, k$ as (1.1.1), which can be seen as meromorphic sections of the fiber bundle $J_{k} X \rightarrow X$. It follows from [Nog86] (see also [NW14, §4.6.3]) that there exists also a natural holomorphic fiber bundle $J_{k}(X, \log D)$ such that

(i) there is a fiber mapping $\lambda: J_{k}(X, \log D) \rightarrow J_{k} X$, locally defined by

$$
\begin{aligned}
\lambda: J_{k}(X, \log D)_{\mid U} & \rightarrow J_{k} X_{\mid U} \\
\left(z ; z_{\ell}^{(j)}, z_{i}^{(j)}\right)_{1 \leqslant j \leqslant k, 1 \leqslant \ell \leqslant c^{\prime}, c^{\prime}<i \leqslant n} & \mapsto\left(z ; z_{\ell} \cdot z_{\ell}^{(j)}, z_{i}^{(j)}\right)_{1 \leqslant j \leqslant k, 1 \leqslant \ell \leqslant c^{\prime}, c^{\prime}<i \leqslant n} ;
\end{aligned}
$$

(ii) the induced mapping between sections of holomorphic fiber bundles

$$
\lambda_{*}: \Gamma\left(U, J_{k}(X, \log D)\right) \rightarrow \mathscr{J}_{k}(X, \log D)(U)
$$

is an isomorphism.

Let us denote by $w_{1}=\log z_{1}, \ldots, w_{c^{\prime}}=\log z_{c^{\prime}}, w_{c^{\prime}+1}=z_{c^{\prime}+1}, \ldots, w_{n}=z_{n}$. The notation $\log z_{i}$ should be understood formally and is used to simplify the notation $d w_{i}=d \log z_{i}=\frac{d z_{i}}{z_{i}}$. One then has another trivialization of $J_{k}(X, \log D)_{\uparrow U}$ is given as follows:

$$
\left(d^{j} w_{i}\right)_{1 \leqslant j \leqslant k, 1 \leqslant i \leqslant n}: J_{k}(X, \log D)_{\mid U} \rightarrow U \times \mathbb{C}^{n k} .
$$

A local meromorphic $k$-jet differential $\alpha$ on $U$ is called a logarithmic $k$-jet differential, if $\alpha(\beta)$ is holomorphic for any $\operatorname{logarithmic} k$-jet field $\beta \in \mathscr{J}_{k}(X, \log D)(U)$. The sheaf of $\operatorname{logarithmic}$ $k$-jet differential is denoted by $\mathcal{E}_{k, \bullet}^{\mathrm{GG}} \Omega_{X}(\log D)$, which is also a locally free sheaf. The associated vector bundle is denoted by $E_{k, \bullet}^{\mathrm{GG}} \Omega_{X}(\log D)$, and is called $k$-jet logarithmic Green-Griffiths bundle. One also the following natural splitting

$$
E_{k, \bullet}^{\mathrm{GG}} \Omega_{X}(\log D)=\bigoplus_{m \geqslant 0} E_{k, m}^{\mathrm{GG}} \Omega_{X}(\log D),
$$

where $E_{k, m}^{\mathrm{GG}} \Omega_{X}(\log D)$ is the logarithmic $k$-jet differentials of weighted degree $m$. Any local section $P \in \mathscr{E}_{k, m} \mathrm{GG} \Omega_{X}(\log D)(U)$ can be written as

$$
\sum_{|\alpha|=m} c_{\alpha}(z)\left(d^{1} w\right)^{\alpha_{1}}\left(d^{2} w\right)^{\alpha_{2}} \cdots\left(d^{k} w\right)^{\alpha_{k}}
$$

where $c_{\alpha}(z) \in \mathscr{O}(U)$ for any $\alpha:=\left(\alpha_{1}, \ldots, \alpha_{k}\right) \in\left(\mathbb{N}^{n}\right)^{k}$. We will use another trivialization of $E_{k, m}^{\mathrm{GG}} \Omega_{X}(\log D)$. First, let us begin with a lemma.

Lemma 1.1. Assume that locally on an open subset of $U \subset X$ with local coordinates $\left(z_{1}, \ldots, z_{n}\right)$ such that $D \cap U=\left(z_{1}=0\right)$. Then for any $j \in \mathbb{N}, \frac{d^{j} z_{1}}{z_{1}}$ is a logarithmic jet differential and 
moreover, any local section $P \in \mathscr{E}_{k, m} \mathrm{GG} \Omega_{X}^{*}(\log D)(U)$ can be written as

$$
\sum_{|\alpha|=m} \frac{c_{\alpha}(z)}{z_{1}^{\alpha_{1}^{1}+\cdots+\alpha_{k}^{1}}}\left(d^{1} z\right)^{\alpha_{1}}\left(d^{2} z\right)^{\alpha_{2}} \cdots\left(d^{k} z\right)^{\alpha_{k}}
$$

where $c_{\alpha}(z) \in \mathscr{O}(U)$ for any $\alpha:=\left(\alpha_{1}, \ldots, \alpha_{k}\right) \in\left(\mathbb{N}^{n}\right)^{k}$ with $\alpha_{j}=\left(\alpha_{j}^{1}, \ldots, \alpha_{j}^{n}\right) \in \mathbb{N}^{n}$.

Proof. Let us prove by induction that for any $j \geqslant 1$ and any $\beta=\left(\beta_{1}, \ldots, \beta_{j}\right) \in \mathbb{N}^{j}$ there exists $b_{j \beta} \in \mathbb{Z}$ such that

$$
\frac{d^{j} z_{1}}{z_{1}}=\sum_{\beta_{1}+2 \beta_{2}+\cdots+j \beta_{j}=j} b_{j \beta} \cdot\left(d^{1} \log z_{1}\right)^{\beta_{1}}\left(d^{2} \log z_{1}\right)^{\beta_{2}} \cdots\left(d^{j} \log z_{1}\right)^{\beta_{j}} .
$$

By definition, it holds for $j=1$. Assume now that (1.2.6) holds for $j$. Then

$$
\begin{aligned}
d^{j+1} z_{1}= & d^{1} z_{1} \cdot \sum_{\beta_{1}+2 \beta_{2}+\cdots+j \beta_{j}=j} b_{j \beta} \cdot\left(d^{1} \log z_{1}\right)^{\beta_{1}}\left(d^{2} \log z_{1}\right)^{\beta_{2}} \cdots\left(d^{j} \log z_{1}\right)^{\beta_{j}}+ \\
& z_{1} \cdot \sum_{\beta_{1}+2 \beta_{2}+\cdots+j \beta_{j}=j} \sum_{i=1}^{j} \beta_{i} b_{j \beta} \cdot\left(d^{1} \log z_{1}\right)^{\beta_{1}} \cdots\left(d^{i} \log z_{1}\right)^{\beta_{i}-1}\left(d^{i+1} \log z_{1}\right)^{\beta_{i+1}+1} \cdots\left(d^{j} \log z_{1}\right)^{\beta_{j}} \\
= & z_{1} \cdot \sum_{\beta_{1}+2 \beta_{2}+\cdots+j \beta_{j}=j} b_{j \beta} \cdot\left(d^{1} \log z_{1}\right)^{\beta_{1}+1}\left(d^{2} \log z_{1}\right)^{\beta_{2}} \cdots\left(d^{j} \log z_{1}\right)^{\beta_{j}}+ \\
& z_{1} \cdot \sum_{\beta_{1}+2 \beta_{2}+\cdots+j \beta_{j}=j} \sum_{i=1}^{j} \beta_{i} b_{j \beta} \cdot\left(d^{1} \log z_{1}\right)^{\beta_{1}} \cdots\left(d^{i} \log z_{1}\right)^{\beta_{i}-1}\left(d^{i+1} \log z_{1}\right)^{\beta_{i+1}+1} \cdots\left(d^{j} \log z_{1}\right)^{\beta_{j}},
\end{aligned}
$$

and thus (1.2.6) holds also for $j+1$.

On the other hand, one will prove by induction on $j$ that

$$
d^{j} \log z_{1}=\sum_{\beta_{1}+2 \beta_{2}+\cdots+j \beta_{j}=j} b_{j \beta} \cdot\left(\frac{d^{1} z_{1}}{z_{1}}\right)^{\beta_{1}}\left(\frac{d^{2} z_{1}}{z_{1}}\right)^{\beta_{2}} \cdots\left(\frac{d^{j} z_{1}}{z_{1}}\right)^{\beta_{j}}
$$

where $b_{j \beta} \in \mathbb{Z}$ and $\beta=\left(\beta_{1}, \ldots, \beta_{j}\right) \in \mathbb{N}^{j}$. Assume that (1.2.7) holds for $j$. Then

$d^{j+1} \log z_{1}=\sum_{\beta_{1}+2 \beta_{2}+\cdots+j \beta_{j}=j} \sum_{i=1}^{j} \beta_{i} b_{j \beta} \cdot\left(\frac{d^{1} z_{1}}{z_{1}}\right)^{\beta_{1}} \cdots\left(\frac{d^{i} z_{1}}{z_{1}}\right)^{\beta_{i}-1} \cdots\left(\frac{d^{j} z_{1}}{z_{1}}\right)^{\beta_{j}}\left(\frac{d^{i+1} z_{1}}{z_{1}}-\frac{d^{i} z_{1} d^{1} z_{1}}{z_{1}^{2}}\right)$.

Hence (1.2.7) also holds for $j+1$. It thus just remains to use (1.2.7) and (1.2.4) to show $(1.2 .5)$.

1.3. Demailly-Semple tower. In this section we recall the formalism of directed pairs as introduced by Demailly [Dem97]. A directed manifold $(X, V)$ is a complex manifold $X$ equipped with a subbundle $V \subset T_{X}$ of rank $r$. A morphism of directed manifolds $f:\left(Y, V_{Y}\right) \rightarrow\left(X, V_{X}\right)$ is by definition a morphism $f: Y \rightarrow X$ such that $f_{*} V_{Y} \subset f^{*} V_{X} \subset f^{*} T_{X}$. In [Dem97] Demailly introduced the 1-jet functor which to any directed manifold $(X, V)$ associates the directed manifold defined by $\mathrm{P}_{1} V=\mathrm{P}(V)$ and $V_{1}:=\left(\pi_{0,1}\right)_{*}^{-1} \mathscr{O}_{\mathrm{P}(V)}(-1) \subset T_{X_{1}}$, where $\mathscr{O}_{\mathrm{P}_{1} V}(-1)$ denotes the tautological line bundle $\mathscr{O}_{\mathrm{P}(V)}(-1)$. This induces a morphism between directed manifolds $\left(\mathrm{P}_{1} V, V_{1}\right) \stackrel{\pi_{0,1}}{\longrightarrow}(X, V)$. By iterating this 1-jet functor, Demailly then constructed the so-called Demailly-Semple k-jet tower

$$
\left(\mathrm{P}_{k} V, V_{k}\right) \stackrel{\pi_{k-1, k}}{\longrightarrow}\left(\mathrm{P}_{k-1} V, V_{k-1}\right) \stackrel{\pi_{k-2, k-1}}{\longrightarrow} \cdots \rightarrow\left(\mathrm{P}_{1} V, V_{1}\right) \stackrel{\pi_{0,1}}{\longrightarrow}(X, V)
$$

such that $\mathrm{P}_{k} V:=\mathrm{P}\left(V_{k-1}\right)$ and $V_{k}:=\left(\pi_{k-1, k}\right)_{*}^{-1} \mathscr{O}_{\mathrm{P}_{k} V}(-1) \subset T_{\mathrm{P}_{k} V}$. Here we denote by $\mathscr{O}_{\mathrm{P}_{k} V}(-1)$ the tautological line bundle $\mathscr{O}_{\mathrm{P}\left(V_{k-1}\right)}(-1), \pi_{k-1, k}: \mathrm{P}_{k} V \rightarrow \mathrm{P}_{k-1} V$ the natural projection and 
$\left(\pi_{k-1, k}\right)_{*}=d \pi_{k-1, k}: T_{\mathrm{P}_{k} V} \rightarrow \pi_{k-1, k}^{*} T_{\mathrm{P}_{k-1} V}$ the differential. By composing the projections we get for all pairs of indices $0 \leqslant j \leqslant k$ natural morphisms

$$
\pi_{j, k}: \mathrm{P}_{k} V \rightarrow \mathrm{P}_{j} V, \quad\left(\pi_{j, k}\right)_{*}=\left(d \pi_{j, k}\right)_{\uparrow V_{k}}: V_{k} \rightarrow\left(\pi_{j, k}\right)^{*} V_{j}
$$

For every $k$-tuple $\left(a_{1}, \ldots, a_{k}\right) \in \mathbb{Z}^{k}$ we write $\mathscr{O}_{\mathrm{P}_{k} V}\left(a_{1}, \ldots, a_{k}\right)=\bigotimes_{1 \leqslant j \leqslant k} \pi_{j, k}^{*} \mathscr{O}_{\mathrm{P}_{j} V}\left(a_{j}\right)$. One can inductively define $k$-th lift $f_{[k]}:(\mathbb{C}, 0) \rightarrow \mathrm{P}_{k} V$ for germs of non-constant holomorphic curves $f:(\mathbb{C}, 0) \rightarrow X$ by $f_{[k]}(t)=\left(f_{[k-1]}(t),\left[f_{[k-1]}^{\prime}(t)\right]\right)$ (although this is not well defined when $f_{k-1}^{\prime}(t)=0$ one can easily extend this definition to every $t$ in the domain of definition of $f$ ).

On the other hand, let $\mathbb{G}_{k}$ be the group of germs of $k$-jets of biholomorphisms of $(\mathbb{C}, 0)$, that is, the group of germs of biholomorphic maps

$$
\varphi: t \mapsto a_{1} t+a_{2} t^{2}+\cdots+a_{k} t^{k}, \quad a_{1} \in \mathbb{C}^{*}, a_{j} \in \mathbb{C}, j>2,
$$

in which the composition law is taken modulo terms $t^{j}$ of degree $j>k$. Then $\mathbb{G}_{k}$ is a $k$ dimensional nilpotent complex Lie group, which admits a natural fiberwise right action on $J_{k} V$. The action consists of reparameterizing $k$-jets of maps $f:(\mathbb{C}, 0) \rightarrow(X, V)$ by a biholomorphic change of parameter $\varphi:(\mathbb{C}, 0) \rightarrow(\mathbb{C}, 0)$ defined by $(f, \varphi) \mapsto f \circ \varphi$. Moreover, if one denotes by

$$
J_{k}^{\mathrm{reg}} V:=\left\{j_{k} f \in J_{k} V \mid f^{\prime}(0) \neq 0\right\}
$$

the space of regular $k$-jets tangent to $V$, there exists a natural morphism

$$
\begin{aligned}
J_{k}^{\mathrm{reg}} V & \rightarrow \mathrm{P}_{k} V \\
j_{k} f & \mapsto f_{[k]}(0)
\end{aligned}
$$

whose image is an open set in $\mathrm{P}_{k} V$ denoted by $\mathrm{P}_{k} V^{\text {reg; }}$ in other words, $\mathrm{P}_{k} V^{\text {reg }} \subset \mathrm{P}_{k} V$ is the set of elements $f_{[k]}(0)$ in $\mathrm{P}_{k} V$ which can be reached by regular germs of curves $f$. It was proved in [Dem97, Theorem 6.8] that $\mathbb{G}_{k}$ acts transitively on $J_{k}^{\text {reg }} V$, and thus $\mathrm{P}_{k} V^{\text {reg }}$ can be identified with the quotient $J_{k}^{\text {reg }} / \mathbb{G}_{k}$. Moreover, the singular $k$-jets, denoted by $\mathrm{P}_{k} V^{\text {sing }}:=\mathrm{P}_{k} V \backslash \mathrm{P}_{k} V^{\text {reg }}$, is a divisor in $\mathrm{P}_{k} V$. In summary, $\mathrm{P}_{k} V$ is a smooth compactification of $J_{k}^{\text {reg }} / \mathbb{G}_{k}$. As will become clear later, and as was observed in [Dem97, §7], when dealing with hyperbolicity questions, the locus $\mathrm{P}_{k} V^{\text {sing }}$ is in some sense irrelevant.

Let us recall the following theorem by Demailly which is a crucial tool in our paper.

Theorem 1.2 ([Dem97, Corollary 5.12, Theorem 6.8]). Let $(X, V)$ be a directed variety.

(i) For any $w_{0} \in \mathrm{P}_{k} V$, there exists an open neighborhood $U_{w_{0}}$ of $w_{0}$ and a family of germs of curves $\left(f_{w}\right)_{w \in U_{w_{0}}}$, tangent to $V$ depending holomorphically on $w$ such that

$$
\left(f_{w}\right)_{[k]}(0)=w \quad \text { and } \quad\left(f_{w}\right)_{[k-1]}^{\prime}(0) \neq 0, \quad \forall w \in U_{w_{0}} .
$$

In particular, $\left(f_{w}\right)_{[k-1]}^{\prime}(0)$ gives a local trivialization of the tautological line bundle $\mathscr{O}_{\mathrm{P}_{k} V}(-1)$ on $U_{w_{0}}$.

(ii) For any $k, m \geqslant 1$ one has

$$
\left(\pi_{0, k}\right)_{*} \mathscr{O}_{\mathrm{P}_{k} V}(m) \cong \mathscr{E}_{k, m} V^{*} .
$$

In fact, the isomorphism (1.3.9) can be understood explicitly in view of Theorem 1.2.(i). With the notation therein, for any given local invariant jet differential $P \in \mathscr{E}_{k, m} V^{*}(U)$, the inverse image under $\left(\pi_{0, k}\right)_{*}$ is the section $\sigma_{P} \in \Gamma\left(U_{w_{0}}, \mathscr{O}_{\mathrm{P}_{k} V}(m)_{\left\lceil U_{w_{0}}\right.}\right)$ defined by

$$
\sigma_{P}(w):=P\left(j_{k} f_{w}\right)\left(\left(f_{w}\right)_{[k-1]}^{\prime}(0)\right)^{-m} .
$$

1.4. Logarithmic Demailly-Semple bundle. In [DL01], Dethloff-Lu extended the DemaillySemple tower to the logarithmic setting. They used it in particular to reprove the Brody hyperbolicity of complements of ample divisors in the abelian varieties. Following [DL01], a logarithmic directed manifold is a triple $(X, D, V)$ where $(X, D)$ is a log manifold, and $V$ is a subbundle of $T_{X}(-\log D)$. In this section, we will recall for the reader's convenience Dethloff-Lu's construction of the logarithmic Demailly(-Semple) $k$-jet tower associated to any logarithmic directed manifold. 
Given log-manifolds $\left(X^{\prime}, D^{\prime}\right)$ and $(X, D)$, a holomorphic map $f: X^{\prime} \rightarrow X$ such that $f^{-1}(D) \subset$ $D^{\prime}$ will be called a log-morphism from $\left(X^{\prime}, D^{\prime}\right)$ to $(X, D)$. It induces morphisms

$$
f_{*}: T_{X^{\prime}}\left(-\log D^{\prime}\right) \rightarrow f^{*} T_{X}(-\log D) \text { and } f^{*}: f^{*} E_{k, m}^{\mathrm{GG}} \Omega_{X}(\log D) \rightarrow E_{k, m}^{\mathrm{GG}} \Omega_{X^{\prime}}\left(\log D^{\prime}\right) .
$$

A $\log$ directed morphism between log directed manifolds $\left(X^{\prime}, D^{\prime}, V^{\prime}\right)$ and $(X, D, V)$ is a $\log$ morphism $f:\left(X^{\prime}, D^{\prime}\right) \rightarrow(X, D)$ such that $f_{*} V^{\prime} \subset V$.

For any fixed order $k$, as the Demailly-Semple bundle, the logarithmic Demailly $k$-jet tower

$$
\left(X_{k}(D), D_{k}, V_{k}\right) \stackrel{\pi_{k-1, k}}{\longrightarrow}\left(X_{k-1}(D), D_{k-1}, V_{k-1}\right) \stackrel{\pi_{k-2, k-1}}{\longrightarrow} \ldots \rightarrow\left(X_{1}(D), D_{1}, V_{1}\right) \stackrel{\pi_{0,1}}{\longrightarrow}(X, D, V)
$$

is constructed inductively. Define $X_{k}(D):=\mathrm{P}\left(V_{k-1}\right)$, and let $\pi_{k-1, k}: X_{k}(D) \rightarrow X_{k-1}(D)$ be the natural projection. Set $D_{k}:=\left(\pi_{k-1, k}\right)^{-1}\left(D_{k-1}\right)$ which is a simple normal crossing divisor, and induces a morphism

$$
\left(\pi_{k-1, k}\right)_{*}: T_{X_{k}(D)}\left(-\log D_{k}\right) \rightarrow\left(\pi_{k-1, k}\right)^{*} T_{X_{k-1}(D)}\left(-\log D_{k-1}\right) .
$$

Define

$$
V_{k}:=\left(\pi_{k-1, k}\right)_{*}^{-1} \mathscr{O}_{X_{k}(D)}(-1) \subset T_{X_{k}(D)}\left(-\log D_{k}\right),
$$

where $\mathscr{O}_{X_{k}(D)}(-1):=\mathscr{O}_{\mathrm{P}\left(V_{k-1}\right)}(-1)$ is the tautological line bundle, which by definition is also a subbundle of $\left(\pi_{k-1, k}\right)^{*} V_{k-1}$. We say that $\left(X_{k}(D), D_{k}, V_{k}\right) \stackrel{\pi_{k-1, k}}{\longrightarrow}\left(X_{k-1}(D), D_{k-1}, V_{k-1}\right)$ is the 1-jet functor of the log direct manifold $\left(X_{k-1}(D), D_{k-1}, V_{k-1}\right)$.

Note that $\operatorname{ker}\left(\pi_{k-1, k}\right)_{*}=T_{X_{k}(D) / X_{k-1}(D)}$ by definition. This gives the following short exact sequence of vector bundles over $X_{k}(D)$

$$
0 \rightarrow T_{X_{k}(D) / X_{k-1}(D)} \rightarrow V_{k} \stackrel{\left(\pi_{k-1, k}\right)_{*}}{\longrightarrow} \mathscr{O}_{X_{k}(D)}(-1) \rightarrow 0 .
$$

Furthermore, we have the Euler exact sequence for projectivized bundles

$$
0 \rightarrow \mathscr{O}_{X_{k}(D)} \rightarrow\left(\pi_{k-1, k}\right)^{*} V_{k-1} \otimes \mathscr{O}_{X_{k}(D)}(1) \rightarrow T_{X_{k}(D) / X_{k-1}(D)} \rightarrow 0
$$

By definition, there is a canonical line bundle morphism

$$
\mathscr{O}_{X_{k}(D)}(-1) \hookrightarrow\left(\pi_{k-1, k}\right)^{*} V_{k-1} \stackrel{\left(\pi_{k-1, k}\right)^{*}\left(\pi_{k-2, k-1}\right)_{*}}{\longrightarrow}\left(\pi_{k-1, k}\right)^{*} \mathscr{O}_{X_{k-1}(D)}(-1)
$$

which admits precisely $\Gamma_{k}:=\mathrm{P}\left(T_{X_{k}(D) / X_{k-1}(D)}\right) \subset \mathrm{P}\left(V_{k}\right)=X_{k}(D)$ as its zero divisor:

$$
\mathscr{O}_{X_{k}(D)}(1)=\left(\pi_{k-1, k}\right)^{*} \mathscr{O}_{X_{k-1}(D)}(1) \otimes \mathscr{O}_{X_{k}(D)}\left(\Gamma_{k}\right)
$$

Let us denote by $\pi_{j, k}: X_{k}(D) \rightarrow X_{j}(D)$ the composition of the projections $\pi_{k} \circ \cdots \circ \pi_{j+1}$. Define $X_{k}(D)^{\text {sing }}:=\bigcup_{2 \leqslant j \leqslant k} \pi_{j, k}{ }^{*}\left(\Gamma_{j}\right)$, and $X_{k}(D)^{\text {reg }}:=X_{k}(D) \backslash X_{k}(D)^{\text {sing }}$.

Definition 1.3. For any open subset $U \subset X$, a logarithmic differential operator $P \in \Gamma\left(U, \mathcal{E}_{k, m}^{\mathrm{GG}} \Omega_{X}(\log D)\right)$ is said to be invariant by reparametrization group $\mathbb{G}_{k}$ if for any $g \in \mathbb{G}_{k}$ and any $j_{k} f \in J_{k} X_{\mid X \backslash D}^{\text {reg }}$, one has

$$
P\left(j_{k}(f \circ g)\right)=g^{\prime}(0)^{m} \cdot P\left(j_{k} f\right) .
$$

Let us define $\mathcal{E}_{k, m} \Omega_{X}(\log D)$ to be the subsheaf of $\mathcal{E}_{k, m}^{\mathrm{GG}} \Omega_{X}(\log D)$ which consists of invariant logarithmic differential operator. The associated vector bundle is denoted by $E_{k, m} \Omega_{X}(\log D)$.

The $\log$ Demailly tower is of great importance in the study of the algebraic degeneracy of entire curves on $X \backslash D$, granting the following direct image formula in [DL01, Proposition 3.9]

$$
\mathscr{E}_{k, m} \Omega(\log D)=\left(\pi_{k}\right)_{*} \mathscr{O}_{X_{k}(D)}(m) .
$$

The following fundamental result shows that the logarithmic jet differentials vanishing along some ample divisor provides obstructions to the existence of entire curves in the complement. 
Theorem 1.4 (Dethloff-Lu, Siu-Yeung). Let $X$ be a smooth complex projective variety with $D \subset X$ a normal crossings divisor on $X$, and $X_{k}(D)$ denotes to be the log Demailly $k$-jet tower of $\left(X, D, T_{X}(-\log D)\right)$. For any non-constant entire curve $f: \mathbb{C} \rightarrow X \backslash D$ avoiding $D$, any ample line bundle $A$ on $X$, any $a_{1}, \ldots, a_{k} \in \mathbb{N}$ and any

$$
\omega \in H^{0}\left(X_{k}(D), \mathscr{O}_{X_{k}(D)}\left(a_{1}, \ldots, a_{k}\right) \otimes\left(\pi_{0, k}\right)^{*} A^{-1}\right),
$$

one has $f_{[k]}(\mathbb{C}) \subseteq(\omega=0)$.

1.5. Jet bundle of a line bundle. We recall here the basic definitions and properties of jet bundles of a line bundle (we refer to [Gro66, §16.7] for a detailed presentation). Let $X$ be a complex manifold, and let $L$ be a line bundle on $X$. For any integer $k \geqslant 0$, on defines the $k$-th order jet bundle $J^{k} L$ of $L$ as follows. Consider the product $X \times X$ with the canonical projections $\mathrm{pr}_{1}, \mathrm{pr}_{2}$ on the first and second factors. Let $\Delta_{X} \subset X \times X$ be the diagonal and $\mathscr{I}_{\Delta_{X}} \subset \mathscr{O}_{X \times X}$ denotes its ideal sheaf. Then one defines

$$
J^{k} L:=\operatorname{pr}_{1 *}\left(\mathscr{O}_{X \times X} / \mathscr{I}_{\Delta_{X}}^{k+1} \otimes \operatorname{pr}_{2}^{*} L\right) .
$$

It can be shown that this is a locally free sheaf on $X$ such that for an $x \in X$, the fiber at $x$ is $J_{x}^{k} L=L \otimes \mathscr{O}_{X, x} / \mathfrak{m}_{X, x}^{k+1}$. This construction is also functorial in the following way: given a complex manifold $Y$ and morphism $\varphi: X \rightarrow Y$, one obtains a natural morphsim of $\mathscr{O}_{X}$-modules

$$
\varphi^{*}: \varphi^{*} J^{k} L \rightarrow J^{k} \varphi^{*} L,
$$

induced by the commutativity of the diagram

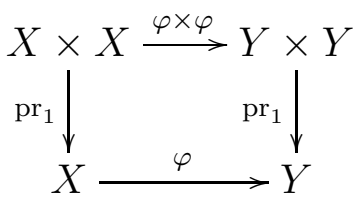

and the fact that $(\varphi \times \varphi)^{-1} \mathscr{I}_{\Delta_{Y}} \subset \mathscr{I}_{\Delta_{X}}$.

We shall need the following elementary proposition.

Proposition 1.5. Let $L$ and $L^{\prime}$ be line bundles on $X$. Any morphism of $\mathscr{O}_{X}$-modules $h: L \rightarrow L^{\prime}$ induces a morphism of $\mathscr{O}_{X}$-modules

$$
J^{k} L \stackrel{j^{k} h}{\rightarrow} J^{k} L^{\prime}
$$

Moreover, $j^{k} h$ is an isomorphism whenever $h$ is an isomorphism.

Proof. The morphism $j^{k} h$ is just the push-forward under $\operatorname{pr}_{1}$ of the morphism

$$
\operatorname{pr}_{2}^{*} h: \mathscr{O}_{X \times X} / \mathscr{I}_{\Delta_{X}} \otimes L \rightarrow \mathscr{O}_{X \times X} / \mathscr{I}_{\Delta_{X}} \otimes L^{\prime}
$$

induced by $h$. The second assertion follows at once.

Observe that there exists a $\mathbb{C}$-linear morphism

$$
j_{L}^{k}: L \rightarrow J^{k} L
$$

which is not a morphism of $\mathscr{O}_{X}$-modules, defined, at the level of presheaves, as the composition, for any open subset $U \subset X$,

$$
L(U) \stackrel{\mathrm{pr}_{2}^{*}}{\rightarrow} \operatorname{pr}_{2}^{*} L(U \times U) \rightarrow \mathscr{O}_{U \times U} / \mathscr{I}_{\Delta}^{k+1} \otimes \operatorname{pr}_{2}^{*} L(U \times U)=J^{k} L(U) .
$$

More explicitly, for any $s \in L(U)$, the section $j_{L}^{k}(s) \in J^{k} L(U)$ is such that for any $x \in U$, the element $j_{L}^{k}(s)(x) \in J_{x}^{k} L=L \otimes \mathscr{O}_{X, x} / \mathfrak{m}_{X, x}^{k+1}$ is precisely the image of $s$ under the map $L(U) \rightarrow L \otimes \mathscr{O}_{X, x} / \mathfrak{m}_{X, x}^{k+1}$.

This map can also be understood more explicitly in coordinates. Take an open subset $U \subset X$, up to considering a trivialization of $L_{\uparrow U}$, one is reduced to understand $\mathscr{O}_{U} \rightarrow J^{k} \mathscr{O}_{U}$. Observe that coordinates $\left(x_{1}, \ldots, x_{n}\right)$ induce coordinates $\left(x_{1}, \ldots, x_{n}, z_{1}, \ldots, z_{n}\right)$ on $U \times U$, from which one obtains that the monomials $\left((z-x)^{I}\right)_{|I| \leqslant k}$ form a local frame for $J^{k} \mathscr{O}_{U}$. Here, we use the 
multi-index notation, $(z-x)^{\alpha}=\left(z_{1}-x_{1}\right)^{\alpha_{1}} \cdots\left(z_{n}-x_{n}\right)^{\alpha_{n}}$ for $\alpha=\left(\alpha_{1}, \ldots, \alpha_{n}\right)$ such that $|\alpha|=\alpha_{1}+\cdots+\alpha_{n} \leqslant k$. The map $j_{\mathscr{O}_{U}}^{k}: \mathscr{O}_{U} \rightarrow J^{k} \mathscr{O}_{U}$ is then just given by computing, in each $x \in U$, the Taylor expansion up to order $k$, namely, for any $f \in \mathscr{O}(U)$,

$$
j_{\mathscr{O}_{U}}^{k}(f)=\sum_{|\alpha| \leqslant k} \frac{1}{\alpha !} \frac{\partial^{|\alpha|} f}{\partial z^{\alpha}}(x)(z-x)^{\alpha} \in J^{k} \mathscr{O}_{U}(U)
$$

The following definition will be used in the sequel.

Definition 1.6. Let $X$ be a complex manifold and let $L$ be a line bundle on $X$. We say that $L$ separates $k$-jets at every point of $X$ if the natural morphism

$$
j_{L}^{k}: H^{0}(X, L) \otimes \mathscr{O}_{X} \rightarrow J^{k} L
$$

is surjective. Observe that this condition is equivalent to the surjectivity, for every $x \in X$ of the natural map

$$
H^{0}(X, L) \otimes \mathscr{O}_{X} \rightarrow L \otimes \mathscr{O}_{X, x} / \mathfrak{m}_{X, x}^{k+1}
$$

Observe that if $L$ is a very ample line bundle on $X$, then $L^{k}$ separates $k$-jets at every point of $X$.

\section{Higher order LOGARITHMIC CONNECTIONS AND LOGARITHMIC WRONSKIANS}

2.1. Wronskians. Let us recall here the Wronskian constructions initiated by the first named author in [Bro17] and later reinterpreted by the second named author in an alternative way in [Den17]. Let $X$ be a complex manifold, and let $L$ be a line bundle on $X$. Let $k \geqslant 1$ be an integer and take global sections $s_{0}, \ldots, s_{k} \in H^{0}(X, L)$. Then for every open subset $U \subset X$ on which $L$ is trivialized, by considering the holomorphic functions $s_{0, U}, \ldots, s_{k, U} \in \mathscr{O}(U)$ associated to $s_{0}, \ldots, s_{k}$ under our choice of trivialization, we consider the Wronskian

$$
W_{U}\left(s_{0}, \ldots, s_{k}\right):=\left|\begin{array}{ccc}
s_{0, U} & \ldots & s_{k, U} \\
d^{1} s_{0, U} & \ldots & d^{1} s_{k, U} \\
\vdots & \ddots & \vdots \\
d^{k} s_{0, U} & \ldots & d^{k} s_{k, U}
\end{array}\right| \in \mathscr{O}\left(p_{k}^{-1}(U)\right) .
$$

Denote by $k^{\prime}=1+2+\cdots+k$. It was established in [Bro17] that $W_{U}\left(s_{0}, \ldots, s_{k}\right) \in \mathscr{E}_{k, k^{\prime}} \Omega_{X}(U)$, and that those locally defined elements glue together into a global section

$$
W_{L}\left(s_{0}, \ldots, s_{k}\right) \in H^{0}\left(X, E_{k, k^{\prime}} \Omega_{X} \otimes L^{k+1}\right)
$$

which is called Wronskian in [Bro17, §2.2]. Moreover, in [Den17], it was proved that there exists a morphism of $\mathscr{O}_{X}$-modules

$$
j^{k} W_{L}: \bigwedge^{k+1} J^{k} L \rightarrow E_{k, k^{\prime}} \Omega_{X} \otimes L^{k+1}
$$

such that for any global section $s_{0}, \ldots, s_{k} \in H^{0}(X, L)$, one has

$$
j^{k} W_{L}\left(j_{L}^{k} s_{0} \wedge \cdots \wedge j_{L}^{k} s_{k}\right)=W_{L}\left(s_{0}, \ldots, s_{k}\right) .
$$

In $\S 2.3$, we will construct a logarithmic counterpart of Wronskians.

2.2. Higher order logarithmic connections. Let $X$ be a complex manifold. Let $L$ be a line bundle on $X$ and suppose that there exists $\sigma \in H^{0}(X, L)$ such that $D=(\sigma=0)$ is a smooth hypersurface of $X$. Then $L$ is endowed with a natural logarithmic connection $\nabla_{D}: L \rightarrow \Omega_{X}(\log D) \otimes L$, with logarithmic poles along $D$, defined by

$$
\nabla_{D} s:=\sigma d\left(\frac{s}{\sigma}\right)={ }_{\mathrm{loc}} d s-s \frac{d \sigma}{\sigma} .
$$


The second equality has to be understood locally, i.e. if the open subset $U$ over which $L$ is trivialized, and if we denote by $s_{U}, \sigma_{U} \in \mathscr{O}(U)$ the holomorphic functions associated to $s, \sigma$, then one sets

$$
\nabla_{D} s_{U}=d s_{U}-s_{U} \frac{d \sigma_{U}}{\sigma_{U}}
$$

This object is well defined since $\frac{s}{\sigma}$ is a meromorphic function on $X$ and that the local description shows that it has logarithmic poles along $D$. Let us mention that in our paper [BD17] we apply this construction to prove Theorem 0.2.

More generally, for every $k \geqslant 0$, one can define a $\mathbb{C}$-linear map $\nabla_{D}^{k}: L \rightarrow E_{k, k}^{\mathrm{GG}} \Omega_{X}(\log D) \otimes L$ by

$$
\nabla_{D}^{k} s:=\sigma d^{k}\left(\frac{s}{\sigma}\right)
$$

Observe that $\nabla_{D}^{0} s=s$, and that $\nabla_{D}^{1} s=\nabla_{D} s$ for any $s$. Moreover, for any $k \geqslant 1$ one has the local inductive description

$$
\nabla_{D}^{k} s={ }_{\text {loc }} d \nabla_{D}^{k-1} s-\nabla_{D}^{k-1} s \cdot \frac{d \sigma}{\sigma} .
$$

We will need the following elementary, yet crucial, observation: for any $k \geqslant 1$, one has

$$
\nabla_{D}^{k} \sigma=0
$$

Lastly let us observe that locally (with the above notation) one can use the Leibniz rule to $s_{U}=\sigma_{U} \frac{s_{U}}{\sigma_{U}}$ to obtain

$$
d^{k} s_{U}=\sum_{i=0}^{k}\left(\begin{array}{l}
k \\
i
\end{array}\right)\left(\nabla_{D}^{i} s_{U}\right) \frac{d^{k-i} \sigma_{U}}{\sigma_{U}} .
$$

While $\nabla_{D}^{k}: L \rightarrow E_{k, k}^{\mathrm{GG}} \Omega_{X}(\log D) \otimes L$ is only $\mathbb{C}$-linear, we have the following proposition.

Proposition 2.1. With the above notation. There exists a morphism of $\mathscr{O}_{X}$-module

$$
j^{k} \nabla_{D}^{k}: J^{k} L \rightarrow \mathscr{E}_{k, k}^{\mathrm{GG}} \Omega_{X}(\log D) \otimes L,
$$

such that $\left(j^{k} \nabla_{D}^{k}\right) \circ j_{L}^{k}=\nabla_{D}^{k}$.

Proof. The $k$-th order jet space of $X \times X$ naturally splits as

$$
J_{k}(X \times X) \cong J_{k} X \times J_{k} X
$$

under the map $j_{k}\left(f_{1}, f_{2}\right) \mapsto\left(j_{k} f_{1}, j_{k} f_{2}\right)$. Let us define, for any $k, m \geqslant 0$ an operator $d_{2}$ : $\mathscr{E}_{k, m} \Omega_{X \times X} \rightarrow \mathscr{E}_{k+1, m+1} \Omega_{X \times X}$ by setting for any open subset $U \subset X \times X$ and any $P \in$ $\Gamma\left(U, \mathscr{E}_{k, m} \mathrm{GG}_{X \times X}\right)$

$$
d_{2} P\left(j_{k}\left(f_{1}, f_{2}\right)\right)=\frac{d}{d t}\left(P\left(\left(f_{1}(0), j_{k-1} f_{2}(t)\right)\right)(0) .\right.
$$

We define for every $k \in \mathbb{N}^{*}$ an $\mathbb{C}$-linear morphism $\nabla_{2}^{k}: \pi_{2}^{*} L \rightarrow \mathscr{E}_{k, k} \mathrm{GG} \Omega_{X \times X}\left(\log \pi_{2}^{-1}(D)\right) \otimes \pi_{2}^{*} L$ inductively by setting

$$
\begin{aligned}
& \nabla_{2}^{0} s=s \\
& \nabla_{2}^{k} s=\pi_{2}^{*} d_{2}\left(\frac{s}{\pi_{2}^{*} \sigma}\right)={ }_{\text {loc }} d_{2} \nabla_{2}^{k-1} s-\nabla_{2}^{k-1} s \frac{d \pi_{2}^{*} \sigma}{\pi_{2}^{*} \sigma}, \text { for any } k \geqslant 0 .
\end{aligned}
$$

As before, the last equality has to be understood locally and one verifies that this is well defined. Observe that for any open subsets $V \subset X \times X$ and $U \subset X$ such that $\pi_{1}(V) \subset U$, and for any $f \in \mathscr{O}(U)$ one has $d_{2} \pi_{1}^{*} f=0$. Therefore for any $s \in \pi_{2}^{*} L(U)$, one has

$$
\nabla_{2}^{k}\left(\pi_{1}^{*} f \cdot s\right)=\pi_{1}^{*} f \cdot \nabla_{2}^{k} s .
$$

We can consider the composition $\operatorname{res}_{\Delta} \circ \nabla_{2}^{k}$ :

$$
\pi_{2}^{*} L \stackrel{\nabla_{2}^{k}}{\rightarrow} \mathscr{E}_{k, k}^{\mathrm{GG}} \Omega_{X \times X}\left(\log \pi_{2}^{-1}(D)\right) \otimes \pi_{2}^{*} L \stackrel{\text { res } \Delta}{\rightarrow} \mathscr{E}_{k, k} \mathrm{GG} \Omega_{\Delta}\left(\log \pi_{2}^{-1}(D)_{\uparrow \Delta}\right) \otimes \pi_{2}^{*} L_{\uparrow \Delta} .
$$


A local computation now proves that for any open subset $U \subset X \times X$ and every element $s \in \Gamma\left(U, \pi_{2}^{*} L \otimes \mathscr{I}_{\Delta}^{k+1}\right)$, one has

$$
\operatorname{res}_{\Delta} \circ \nabla_{2}^{k}(s)=0
$$

Therefore we obtain a $\mathbb{C}$-linear map

$$
\nabla_{2 \Delta}^{k}: \pi_{2}^{*} L \times \mathscr{O}_{X \times X} / \mathscr{I}_{\Delta}^{k+1} \rightarrow \mathscr{E}_{k, k} \mathrm{GG}_{\Delta}\left(\log \pi_{2}^{-1}(D)_{\uparrow \Delta}\right) \otimes \pi_{2}^{*} L_{\uparrow \Delta},
$$

and by applying $\pi_{1 *}$, we obtain a $\mathbb{C}$-linear morphism

$j^{k} \nabla_{D}^{k}: J^{k} L=\pi_{1 *}\left(\pi_{2}^{*} L \times \mathscr{O}_{X \times X} / \mathscr{I}_{\Delta}^{k+1}\right) \rightarrow \pi_{1 *}\left(\mathscr{E}_{k, k}^{\mathrm{GG}} \Omega_{\Delta}\left(\log \pi_{2}^{-1}(D)_{\uparrow \Delta}\right) \otimes \pi_{2}^{*} L_{\uparrow \Delta}\right) \stackrel{\iota}{\cong} \mathscr{E}_{k, k}^{\mathrm{GG}} \Omega_{X}(\log D) \otimes L$.

Let us now prove that $j^{k} \nabla_{D}^{k}$ is $\mathscr{O}_{X}$-linear. Take an open subset $U \subset X$ and elements $f \in$ $\mathscr{O}(U), \tau \in J^{k} L(U)$. By definition, one can consider (up to shrinking $U$ if necessary) $\tau$ as an element $\tau \in \pi_{2}^{*} L \otimes \mathscr{O}_{X \times X} / \mathscr{I}_{\Delta}^{k+1}\left(\pi_{1}^{-1}(U)\right)$. Up to shrinking $U$ if necessary, take $\tilde{\tau} \in \pi_{2}^{*} L(V)$ representing $\tau$ for some neighborhood $V \subset X \times X$ of $\Delta \cap \pi_{1}^{-1}(U)$. By definition,

$j^{k} \nabla_{D}^{k}(f \tau)=\iota \nabla_{2 \Delta}^{k}\left(\pi_{1}^{*} f \tilde{\tau}\right)=\iota \operatorname{ores}_{\Delta} \circ \nabla_{2}^{k}\left(\pi_{1}^{*} f \cdot \tilde{\tau}\right)=\iota \operatorname{ores}_{\Delta} \circ\left(\pi_{1}^{*} f \cdot \nabla_{2}^{k}(\tilde{\tau})\right)=\pi_{1}^{*} f \cdot \iota \circ \operatorname{res}_{\Delta} \nabla_{2}^{k}(\tilde{\tau})=f \cdot j^{k} \nabla_{D}^{k}(\tau)$.

To see that $\nabla_{D}^{k}=j^{k} \nabla_{D}^{k} \circ j_{L}^{k}$, it suffices, by definition of $j_{L}^{k}$, to prove that for any open subset $U \subset X$ and every element $s \in L(U)$, one has

$$
\iota \circ \operatorname{res}_{\Delta} \circ \nabla_{2}^{k}\left(\pi_{2}^{*} s\right)=\nabla_{D}^{k}(s)
$$

But observe that $\nabla_{2}^{k}\left(\pi_{2}^{*} s\right)=\pi_{2, k}^{*} \nabla^{k} s$, where

$$
\pi_{2, k}^{*}: \pi^{*}\left(\mathscr{E}_{k, k}^{\mathrm{GG}} \Omega_{X}(\log D) \otimes L\right) \rightarrow \mathscr{E}_{k, k} \mathrm{GG} \Omega_{X \times X}\left(\log \left(\pi_{2}^{-1} D\right)\right) \otimes \pi_{2}^{*} L
$$

is the map induced by $\pi_{2}$. Moreover, if one denotes $\sigma_{1}: X \rightarrow \Delta$ the canonical lift, one has by definition that $\iota=\sigma_{1, k}^{*}$ is just the isomorphism induced by $\sigma_{1}$ and that therefore $\iota \circ \operatorname{res}_{\Delta} \circ \pi_{2, k}^{*}=\left(\pi_{2} \circ \sigma_{1}\right)_{k}^{*}=\left(\operatorname{id}_{X}\right)_{k}^{*}$ is just the identity on $\mathscr{E}_{k, k} \mathrm{GG} \Omega_{X}(U)$.

2.3. Logarithmic Wronskians. Let $X$ be an $n$-dimensional complex manifold endowed with a line bundle $L$. Suppose that there exists a smooth hypersurface $D \in|L|$ defined by a section $\sigma_{D} \in H^{0}(X, L)$. Fix a positive integer $k \geqslant 1$. Given $s_{1}, \ldots, s_{k} \in H^{0}(X, L)$ we define the logarithmic Wronskian to be

$$
W_{D}\left(s_{1}, \ldots, s_{k}\right):=\left|\begin{array}{ccc}
\nabla_{D}^{1}\left(s_{1}\right) & \cdots & \nabla_{D}^{1}\left(s_{k}\right) \\
\vdots & \ddots & \vdots \\
\nabla_{D}^{k}\left(s_{1}\right) & \cdots & \nabla_{D}^{k}\left(s_{k}\right)
\end{array}\right| \in H^{0}\left(X, E_{k, k^{\prime}}^{\mathrm{GG}} \Omega_{X}(\log D) \otimes L^{k}\right) .
$$

We shall shortly see that in fact these elements define in fact global sections of $E_{k, k^{\prime}} \Omega_{X}(\log D) \otimes$ $L^{k}$. We also define a morphism of $\mathscr{O}_{X}$-modules

$$
j^{k} W_{D}: \bigwedge^{k} J^{k} L \rightarrow E_{k, k^{\prime}}^{\mathrm{GG}} \Omega_{X}(\log D) \otimes L^{k}
$$

by setting

$$
j^{k} W_{D}\left(g_{1} \wedge \cdots \wedge g_{n}\right)=\left|\begin{array}{ccc}
j^{1} \nabla_{D}^{1}\left(g_{1}\right) & \cdots & j^{1} \nabla_{D}^{1}\left(g_{k}\right) \\
\vdots & \ddots & \vdots \\
j^{k} \nabla_{D}^{k}\left(g_{1}\right) & \cdots & j^{k} \nabla_{D}^{k}\left(g_{k}\right)
\end{array}\right|
$$

for $g_{1}, \ldots, g_{k} \in J^{k} L(U)$. Here we use for any $1 \leqslant j \leqslant k$ the inclusion $E_{j, m}^{\mathrm{GG}} \Omega_{X}(\log D) \subset$ $E_{k, m}^{\mathrm{GG}} \Omega_{X}(\log D)$ and the truncating morphism $J^{k} L \rightarrow J^{j} L$. This construction is related to the (non-logarithmic) Wronskian in the following way.

Lemma 2.2. Same notation as above. For any open subset $U \subset X$ and any $g_{1}, \ldots, g_{k} \in L(U)$ one has

$$
j^{k} W_{L}\left(j_{L}^{k} \sigma_{D} \wedge j_{L}^{k} g_{1} \wedge \cdots \wedge j_{L}^{k} g_{k}\right)=\sigma_{D} \cdot j^{k} W_{D}\left(j_{L}^{k} g_{1} \wedge \cdots \wedge j_{L}^{k} g_{k}\right)
$$


Proof. On proves by using elementary operations on the lines and Leibniz relation (2.2.5), that for any $g_{0}, \ldots, g_{k} \in L(U)$ one has

$$
j^{k} W_{L}\left(j_{L}^{k} g_{0} \wedge \cdots \wedge j_{L}^{k} g_{k}\right)=\left|\begin{array}{ccc}
j^{0} \nabla_{D}^{0}\left(j_{L}^{0} g_{0}\right) & \cdots & j^{0} \nabla_{D}^{0}\left(j_{L}^{0} g_{k}\right) \\
\vdots & \ddots & \vdots \\
j^{k} \nabla_{D}^{k}\left(j_{L}^{k} g_{0}\right) & \cdots & j^{k} \nabla_{D}^{k}\left(j_{L}^{k} g_{k}\right)
\end{array}\right| .
$$

Then one applies this equality to $g_{0}=\sigma_{D}$ and use relation (2.2.4) and Proposition 2.1 to prove that for any $p \geqslant 1, j^{p} \nabla_{D}^{p}\left(j^{p} \sigma_{D}\right)=0$. The lemma follows by expanding the determinant with respect to the first column.

In particular, we see that the morphism $j^{k} W_{D}$ factors through a morphism

$$
\bigwedge^{k} J^{k} L \rightarrow E_{k, k^{\prime}} \Omega_{X}(\log D) \otimes L^{k} \hookrightarrow E_{k, k^{\prime}}^{\mathrm{GG}} \Omega_{X}(\log D) \otimes L^{k}
$$

which we shall denote (slightly abusively) by $j^{k} W_{D}$ in the rest of this paper. Therefore, by Proposition 2.1 we obtain also that for any $s_{1}, \ldots, s_{k} \in H^{0}(X, L)$,

$$
W_{D}\left(s_{1}, \ldots, s_{k}\right) \in H^{0}\left(X, E_{k, k^{\prime}} \Omega_{X}(\log D) \otimes L^{k}\right) .
$$

By (1.4.13), there exists a unique global section

$$
\omega_{D}\left(s_{1}, \ldots, s_{k}\right) \in H^{0}\left(X_{k}(D), \mathscr{O}_{X_{k}(D)}\left(k^{\prime}\right) \otimes \pi_{0, k}^{*} L^{k}\right)
$$

such that $\left(\pi_{0, k}\right)_{*} \omega_{D}\left(s_{1}, \ldots, s_{k}\right)=W_{D}\left(s_{1}, \ldots, s_{k}\right)$. These observations will be refined even further in the next section.

If $Y$ is a submanifold of $X$ which is transverse to $D$, then $(Y, Y \cap D)$ is a sub-log manifold of $(X, D)$. Write $D_{Y}:=Y \cap D$. One has the following commutative diagram

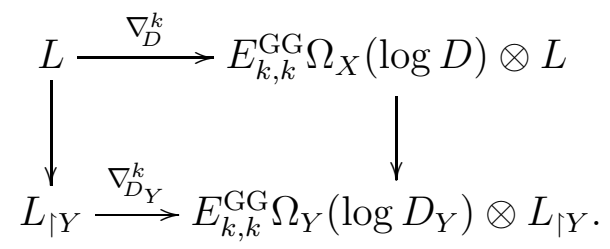

In particular, for any $s_{1}, \ldots, s_{k} \in H^{0}(X, L)$, one has

$$
W_{D}\left(s_{1}, \ldots, s_{k}\right)_{\uparrow Y}=W_{D_{Y}}\left(s_{1 \uparrow Y}, \ldots, s_{k \uparrow Y}\right) .
$$

Since the log Demailly $k$-jet tower $Y_{k}\left(D_{Y}\right)$ of $(Y, D)$ can be seen as a smooth subvariety of $X_{k}(D)$, it follows that

$$
\omega_{D}\left(s_{1}, \ldots, s_{k}\right)_{\uparrow Y_{k}\left(D_{Y}\right)}=\omega_{D_{Y}}\left(s_{1 \uparrow Y}, \ldots, s_{k \uparrow Y}\right) .
$$

2.4. Higher order log connections as local functions on the log Demailly tower. Take $X, L$ and $D$ as in the previous subsection. Fix a positive integer $k \geqslant 1$. Consider the $\log$ Demailly $k_{\text {-jet tower }} X_{k}(D)$ associated to $\left(X, D, T_{X}(-\log D)\right)$. Recall that given any $s_{1}, \ldots, s_{k} \in H^{0}(X, L)$, one can associate to $W_{D}\left(s_{1}, \ldots, s_{k}\right)$ a unique element $\omega_{D}\left(s_{1}, \ldots, s_{k}\right) \in$ $H^{0}\left(X_{k}(D), \mathscr{O}_{X_{k}(D)}\left(k^{\prime}\right) \otimes \pi_{0, k}^{*} L^{k}\right)$ by (2.3.8). The drawback of using (1.4.13) is that the element $\omega_{D}\left(s_{1}, \ldots, s_{k}\right)$ is not fully explicit, since the isomorphism in loc. cit. is not completely explicit. To be more precise, on $X \backslash D$ this isomorphism coincides with (1.3.9), and can therefore be understood in view of Theorem 1.2.(i) and (1.3.10). However, (1.4.13) is only obtained indirectly in a neighborhood of a point of $D$. On the other hand, during the proof of our main result, we will need an explicit description of $\omega_{D}\left(s_{1}, \ldots, s_{k}\right)$ at every point. For this reason, we provide here an alternative way, closer to Demailly's philosophy of directed pairs, to describe this element. To be more precise, we will construct an element

$$
\omega_{D}^{\prime}\left(s_{1}, \ldots, s_{k}\right) \in H^{0}\left(X_{k}(D), \mathscr{O}_{X_{k}(D)}(k, k-1, \ldots, 1) \otimes \pi_{0, k}^{*} L\right)
$$


which is sent to $\omega_{D}\left(s_{1}, \ldots, s_{k}\right)$ under the canonical inclusion $\mathscr{O}_{X_{k}(D)}(k, k-1, \ldots, 1) \hookrightarrow \mathscr{O}_{X_{k}(D)}\left(k^{\prime}\right)$ induced by multiplication by

$$
k \pi_{2, k}^{*} \Gamma_{2}+(k+k-1) \pi_{3, k}^{*} \Gamma_{3}+\cdots+(k+\cdots+3) \pi_{k-1, k}^{*} \Gamma_{k-1}+(k+\cdots+2) \Gamma_{k},
$$

where $\Gamma_{j} \in H^{0}\left(X_{j}(D), \mathscr{O}_{X_{j}(D)}(1) \otimes \pi_{j-1, j}^{*} \mathscr{O}_{X_{j-1}(D)}(-1)\right)$ is the effective divisor defined in (1.4.12).

The starting point of our construction is the following. Let $(X, D, V)$ be a log directed manifold, and let $(\widetilde{X}, \widetilde{D}, \widetilde{V})$ be the derived log directed manifold via the 1-jet functor as defined in $\S$ 1.4. Consider open subsets $U \subset X$ and $\widetilde{U} \subset \widetilde{X}$ such that $\tilde{\pi}(\widetilde{U}) \subset U$. Suppose that we are given a trivialization of $\mathscr{O}_{\widetilde{X}}(-1)_{\uparrow \widetilde{U}}$ induced by a nowhere vanishing section $\xi \in \Gamma\left(\widetilde{U}, \mathscr{O}_{\widetilde{X}}(-1)\right)$ and suppose moreover, that we are given a trivialization of $L_{\uparrow U}$ under which the section $\sigma_{D}$ corresponds to a holomorphic function $\sigma_{U} \in \mathscr{O}(U)$. Then, given any $f \in \mathscr{O}(U)$, we can define

$$
\nabla_{U} f=d f-f \frac{d \sigma_{U}}{\sigma_{U}} \in \Gamma\left(U, \Omega_{X}(D)\right)
$$

Thus we obtain an element $\tilde{\pi}^{*} \nabla_{U} f_{\Gamma \widetilde{U}} \in \Gamma\left(\widetilde{U}, \tilde{\pi}^{*} \Omega_{X}(\log D)\right)$. Note that since we have inclusions

$$
\mathscr{O}_{\widetilde{X}}(-1) \hookrightarrow \tilde{\pi}^{*} V \hookrightarrow \tilde{\pi}^{*} T X(-\log D),
$$

we can see $\xi$ as an element in $\Gamma\left(\widetilde{U}, \tilde{\pi}^{*} T X(-\log D)\right)$. Therefore we can define $\nabla_{U, \sigma_{U}, \widetilde{U}, \xi}^{\mathrm{DS}}(f) \in$ $\mathscr{O}(\widetilde{U})$ by setting

$$
\nabla_{U, \sigma_{U}, \widetilde{U}, \xi}^{\mathrm{DS}}(f)(a)=\tilde{\pi}^{*} \nabla_{U} f_{\uparrow \widetilde{U}}(\xi(a)) \quad \forall a \in \tilde{U} .
$$

Observe that this $\tilde{f}$ depends strongly on the choice of trivializations.

This procedure can now be extended by induction on the higher order log Demailly tower. Consider a log-manifold $(X, D)$ and write $L=\mathscr{O}_{X}(D)$. Consider the log Demailly tower $\left(X_{k}(D), D_{k}, V_{k}\right)_{k \geqslant 0}$ associated to the log directed manifold $\left(X_{0}, D_{0}, V_{0}\right)=\left(X, D, T_{X}(-\log D)\right)$. A trivialization tower of oder $k, \mathfrak{U}=\left(\left(U_{0}, \sigma_{U}\right),\left(U_{j}, \xi_{j}\right)_{1 \leqslant j \leqslant k}\right)$, consists of the following data:

(i) An open subset $U \subset X$ and for each $1 \leqslant j \leqslant k$, an open subset $U_{j} \subset X_{j}(D)$ such that $\pi_{0,1}\left(U_{1}\right) \subset U$ and $\pi_{j, j+1}\left(U_{j+1}\right) \subset U_{j}$ whenever $j<k$.

(ii) A trivialization of $L_{\uparrow U}$ under which the section $\sigma_{D}$ corresponds to a holomorphic function $\sigma_{U} \in \mathscr{O}\left(U_{0}\right)$.

(iii) For every $1 \leqslant j \leqslant k$, a nowhere vanishing section $\xi_{j} \in \Gamma\left(U_{j}, \mathscr{O}_{X_{j}(D)}(-1)\right)$ which therefore induces a trivialization of $\mathscr{O}_{X_{j}(D)}(-1)_{\uparrow U_{j}}$.

Let $\mathfrak{U}=\left(\left(U, \sigma_{U}\right),\left(U_{j}, \xi_{j}\right)_{1 \leqslant j \leqslant k}\right)$ be a trivialization tower of order $k$ and let $f \in \mathscr{O}(U)$ be a holomorphic function on $U$. Then on can define for any $0 \leqslant j \leqslant k$, a holomorphic function

$$
\nabla_{\mathfrak{U}}^{j} f \in \mathscr{O}\left(U_{j}\right)
$$

inductively by setting

$$
\begin{aligned}
\nabla_{\mathfrak{U}}^{0} f & =f \\
\nabla_{\mathfrak{U}}^{j+1} f & =\nabla_{U_{j}, \pi_{0, j}^{*} \sigma_{U}, U_{j+1}, \xi_{j+1}}^{\mathrm{DS}}\left(\nabla_{\mathfrak{U}}^{j} f\right) \quad \forall 0 \leqslant j<k,
\end{aligned}
$$

where we observe that $\left(\pi_{0, j}^{*} \sigma_{U}=0\right) \cap U_{j}$ defines $D_{j} \cap U_{j}$. Here again these functions all depend in a critical way of the choice of trivialization tower $\mathfrak{U}$.

Consider now global sections $s_{1}, \ldots, s_{k} \in H^{0}(X, L)$. Let us fix a trivialization tower $\mathfrak{U}$ and let $s_{1, U}, \ldots, s_{k, U} \in \mathscr{O}(U)$ be the local representatives of $s_{1}, \ldots, s_{k}$ under our choice of trivialization for $L_{\uparrow U}$ (i.e. $s_{i, U}=\sigma_{U} \frac{s_{i}}{\sigma_{D}}$ ) and define

$$
\omega_{\mathfrak{U}}\left(s_{1}, \ldots s_{k}\right)=\left|\begin{array}{ccc}
\nabla_{\mathfrak{U}}^{1} s_{1, U} & \cdots & \nabla_{\mathfrak{U}}^{1} s_{k, U} \\
\vdots & & \vdots \\
\nabla_{\mathfrak{U}}^{k} s_{1, U} & \cdots & \nabla_{\mathfrak{U}}^{k} s_{k, U}
\end{array}\right| \in \mathscr{O}\left(U_{k}\right) .
$$


Here we abusively write $\nabla_{\mathfrak{U}}^{j} s_{i, U}$ instead of $\pi_{j, k}^{*} \nabla_{\mathfrak{U}}^{j} s_{i, U} \in \mathscr{O}\left(U_{k}\right)$ for any $1 \leqslant j<k$. The key point is that these locally defined objects can be glued together.

Proposition 2.3. For any $s_{1}, \ldots, s_{k} \in H^{0}(X, L)$, the family of holomorphic functions $\left(\omega_{\mathfrak{U}}\left(s_{1}, \ldots, s_{k}\right)\right)_{\mathfrak{U}}$ define a global section

$$
\omega_{D}^{\prime}\left(s_{1}, \ldots, s_{k}\right) \in H^{0}\left(X_{k}(D), \mathscr{O}_{X_{k}(D)}(k, k-1, \ldots, 1) \otimes \pi_{0, k}^{*} L^{k}\right) .
$$

More precisely, for any trivialization tower $\mathfrak{U}$ of order $k$, one has

$$
\omega_{D}^{\prime}\left(s_{1}, \ldots, s_{k}\right)_{\mid U_{k}}=\omega_{\mathfrak{U}}\left(s_{1}, \ldots s_{k}\right) \cdot\left(\pi_{1, k}\right)^{*} \xi_{1}^{-k} \cdot\left(\pi_{2, k}\right)^{*} \xi_{2}^{-(k-1)} \cdots\left(\pi_{k-1, k}\right)^{*} \xi_{k-1}^{-2} \cdot \xi_{k}^{-1} .
$$

Moreover, under the natural inclusion

$$
\begin{aligned}
H^{0}\left(X_{k}(D), \mathscr{O}_{X_{k}(D)}(k, k-1, \ldots, 1) \otimes \pi_{0, k}^{*} L^{k}\right) & \hookrightarrow H^{0}\left(X_{k}(D), \mathscr{O}_{X_{k}(D)}\left(k^{\prime}\right) \otimes \pi_{0, k}^{*} L^{k}\right) \\
& \stackrel{(1.4 .13)}{\simeq} H^{0}\left(X, E_{k, k^{\prime}} \Omega_{X}(\log D) \otimes L^{k}\right),
\end{aligned}
$$

the element $\omega_{D}^{\prime}\left(s_{1}, \ldots, s_{k}\right)$ is sent to $W_{D}\left(s_{1}, \ldots, s_{k}\right)$.

The proof of this result relies on the following technical lemma.

Lemma 2.4. For any trivializing tower $\mathfrak{U}=\left(\left(U, \sigma_{U}\right),\left(U_{j}, \xi_{j}\right)_{1 \leqslant j \leqslant k}\right)$ and any integers $1 \leqslant j<$ $p \leqslant k$, there exists a holomorphic function $\beta_{j, p} \in \mathscr{O}\left(U_{p+1}\right)$ such that for any $f \in \mathscr{O}(U)$ one has

$$
\nabla_{\left(U_{p}, \pi_{p}^{*} \sigma_{U}, U_{p+1}, \xi_{p+1}\right)}^{\mathrm{DS}}\left(\pi_{j, p}^{*} \nabla_{\mathfrak{U}}^{j}(f)\right)=\beta_{j, p} \cdot \pi_{j+1, p+1}^{*} \nabla_{\mathfrak{U}}^{j+1}(f)_{\mid U_{p+1}} .
$$

Proof. By definition of the log Demailly jet tower, the differential of the map $\pi_{j, p}$ induces a morphism

$$
d \pi_{j, p}: \mathscr{O}_{X_{p+1}(D)}(-1) \rightarrow \pi_{j+1, p+1}^{*} \mathscr{O}_{X_{j+1}(D)}(-1)
$$

Over the open subset $U_{p+1}$, since $\xi_{j+1}$ is nowhere vanishing on $U_{j+1}$, there exists $\beta_{j, p} \in \mathscr{O}\left(U_{p+1}\right)$ such that

$$
d \pi_{j, p}\left(\xi_{p+1}\right)=\beta_{j, p} \pi_{j+1, p+1}^{*}\left(\xi_{j+1}\right) .
$$

Let us write $g=\nabla_{\mathfrak{U}}^{j}(f) \in \mathscr{O}\left(U_{j}\right)$ for simplicity, so that by definition (2.4.11)

$$
\nabla_{\mathfrak{U}}^{j+1}(f)=\nabla_{U_{j}, \pi_{0, j}^{*} \sigma_{U}, U_{j+1}, \xi_{j+1}}^{\mathrm{DS}}(g)=\pi_{j, j+1}^{*}\left(d g-g \frac{d \pi_{0, j}^{*} \sigma_{U}}{\pi_{0, j}^{*} \sigma_{U}}\right)\left(\xi_{j+1}\right) .
$$

The proof of the lemma is then reduced to the following computation:

$$
\begin{aligned}
\nabla_{\left(U_{p}, \pi_{p}^{*} \sigma_{U}, U_{p+1}, \xi_{p+1}\right)}^{\mathrm{DS}}\left(\pi_{j, p}^{*} g\right) & =\pi_{p, p+1}^{*}\left(d \pi_{j, p}^{*} g-\pi_{j, p}^{*} g \frac{d \pi_{0, p}^{*} \sigma_{U}}{\pi_{0, p}^{*} \sigma_{U}}\right)\left(\xi_{p+1}\right) \\
& =\pi_{p, p+1}^{*}\left(\pi_{j, p}^{*} d g-\pi_{j, p}^{*} g \cdot \pi_{j, p}^{*} \frac{d \pi_{0, j}^{*} \sigma_{U}}{\pi_{0, j}^{*} \sigma_{U}}\right)\left(\xi_{p+1}\right) \\
& =\left[\pi_{j+1, p+1}^{*} \pi_{j, j+1}^{*}\left(d g-g \frac{d \pi_{0, j}^{*} \sigma_{U}}{\pi_{0, j}^{*} \sigma_{U}}\right)\right]\left(\xi_{p+1}\right) \\
& =\pi_{j+1, p+1}^{*} \pi_{j, j+1}^{*}\left[\left(d g-g \frac{d \pi_{0, j}^{*} \sigma_{U}}{\pi_{0, j}^{*} \sigma_{U}}\right)\left(d \pi_{j, p}\left(\xi_{p+1}\right)\right)\right] \\
& =\pi_{j+1, p+1}^{*}\left[\pi_{j, j+1}^{*}\left(d g-g \frac{d \pi_{0, j}^{*} \sigma_{U}}{\pi_{0, j}^{*} \sigma_{U}}\right)\right]\left(\beta_{j, p} \xi_{j+1}\right) \\
& =\beta_{j, p} \pi_{j+1, p+1}^{*} \nabla_{\mathfrak{U}}^{j+1}(f) .
\end{aligned}
$$

Proof of Proposition 2.3. Consider two trivialization towers

$$
\mathfrak{U}^{1}=\left(\left(U^{1}, \sigma_{U^{1}}\right),\left(U_{j}^{1}, \xi_{j}^{1}\right)_{1 \leqslant j \leqslant k}\right) \text { and } \mathfrak{U}^{2}=\left(\left(U^{2}, \sigma_{U^{2}}\right),\left(U_{j}^{2}, \xi_{j}^{2}\right)_{1 \leqslant j \leqslant k}\right) .
$$

Writing $U^{12}=U^{1} \cap U^{2}$ Let $g \in \mathscr{O}\left(U^{12}\right)$ be the transition map from $U^{2}$ to $U^{1}$ induced by our choice of trivializations for $L$, so that for any global section $s \in H^{0}(X, L)$,

$$
s_{U^{1}\left\lceil U^{12}\right.}=g \cdot s_{U^{2}\left\lceil U^{12}\right.} .
$$


For any $1 \leqslant j \leqslant k$, let us also write $U_{j}^{12}=U_{j}^{1} \cap U_{j}^{2}$ and consider the function $\theta_{j} \in \mathscr{O}\left(U_{j}^{12}\right)$ such that

$$
\xi_{j}^{1}=\theta_{j} \xi_{j}^{2}
$$

Therefore $\theta_{j}$ is the transition map from $U_{j}^{2}$ to $U_{j}^{1}$ for the trivializations $\mathscr{O}_{X_{j}(D)}(1)_{\uparrow U_{j}^{2}} \cong U_{j}^{2} \times \mathbb{C}$ and $\mathscr{O}_{X_{j}(D)}(1)_{\left\lceil U_{j}^{1}\right.} \cong U_{j}^{1} \times \mathbb{C}$ induced by $\xi_{j}^{2}$ and $\xi_{j}^{1}$ respectively. We are now going to establish that for any $0 \leqslant p \leqslant k$, and for any $0 \leqslant j<p$, there exists $P_{j}^{p} \in \mathscr{O}\left(U^{p}\right)$ a holomorphic function such that for any $s \in H^{0}(X, L)$ one has

$$
\nabla_{\mathfrak{U}^{1}}^{p}\left(s_{U^{1}}\right)=\theta_{p} \theta_{p-1} \cdots \theta_{1} g \nabla_{\mathfrak{U}^{2}}^{p}\left(s_{U^{2}}\right)+\sum_{j=0}^{p-1} P_{j}^{p} \nabla_{\mathfrak{U}^{2}}^{j}\left(s_{U^{2}}\right) .
$$

The key point in this formula is that $P_{j}^{p}$ does not depend on $s$. From this, and from elementary operations on the lines in the determinant defining $\omega_{\mathfrak{U}^{i}}\left(s_{1}, \ldots, s_{k}\right)$, it will follow that

$$
\omega_{\mathfrak{U}^{1}}\left(s_{1}, \ldots, s_{k}\right)_{\left\lceil U_{k}^{12}\right.}=\theta_{k} \theta_{k-1}^{2} \cdots \theta_{2}^{k-1} \theta_{1}^{k} g \omega_{\mathfrak{U}^{2}}\left(s_{1}, \ldots, s_{k}\right)_{\left\lceil U_{k}^{12}\right.}
$$

which concludes the proof of the first statement of the proposition.

We will establish (2.4.14) by induction on $p$. For $p=0$ this is just (2.4.13). Take $0 \leqslant p<k$ and suppose that formula (2.4.14) holds for $p$. Take $s \in H^{0}(X, L)$. Recall that

$$
\nabla_{\mathfrak{U}^{1}}^{p+1}\left(s_{U^{1}}\right)=\nabla_{U_{p}^{12}, \pi_{0, p}^{*} \sigma_{U^{1}}, U_{p+1}^{12}, \xi_{p+1}^{1}}^{\mathrm{DS}}\left(\nabla_{\mathfrak{U}^{1}}^{p}\left(s_{U^{1}}\right)\right) .
$$

On the other hand, one has

$$
\begin{aligned}
\nabla_{U_{p}^{12}, \pi_{0, p}^{*} \sigma_{U^{1}}, U_{p+1}^{12}, \xi_{p+1}^{1}}^{\mathrm{DS}}\left(\nabla_{\mathfrak{U}^{1}}^{p}\left(s_{U^{1}}\right)\right) & =\nabla_{U_{p}^{12}, \pi_{0, p}^{*}\left(g \sigma_{U^{2}}\right), U_{p+1}^{12}, \theta_{p+1} \xi_{p+1}^{2}}^{\mathrm{DS}}\left(\nabla_{\mathfrak{U}^{1}}^{p}\left(s_{U^{1}}\right)\right) \\
& =\theta_{p+1} \nabla_{U_{p}^{12}, \pi_{0, p}^{*}\left(g \sigma_{U^{2}}\right), U_{p+1}^{12}, \xi_{p+1}^{2}}^{\mathrm{DS}}\left(\nabla_{\mathfrak{U}^{1}}^{p}\left(s_{U^{1}}\right)\right) \\
& =\theta_{p+1} \nabla_{U_{p}^{12}, \pi_{0, p}^{*} \sigma_{U^{2}}, U_{p+1}^{12}, \xi_{p+1}^{2}}^{\mathrm{DS}}\left(\nabla_{\mathfrak{U}^{1}}^{p}\left(s_{U^{1}}\right)\right)-\theta_{p+1} \nabla_{\mathfrak{U}^{1}}^{p}\left(s_{U^{1}}\right) \pi_{p, p+1}^{*} \frac{d \pi_{0, p}^{*} g}{\pi_{0, p}^{*} g}\left(\xi_{p+1}^{2}\right)
\end{aligned}
$$

Observe that, using our induction hypothesis, the term $-\theta_{p+1} \nabla_{\mathfrak{U}^{1}}^{p}\left(s_{U^{1}}\right) \pi_{p, p+1}^{*} \frac{d \pi_{0, p}^{*} g}{\pi_{0, p}^{*} g}\left(\xi_{p+1}^{2}\right)$ is of the form allowed in formula (2.4.14) to be considered as an error term for the rank $p+1$. Therefore it only remains to prove that $\theta_{p+1} \nabla_{U_{p}^{12}, \pi_{0, \sigma^{2}}^{*}, U_{p+1}^{12}, \xi_{p+1}^{2}}^{\mathrm{DS}}\left(\nabla_{\mathfrak{U}^{1}}^{p}\left(s_{U^{1}}\right)\right)$ is of the form announced in (2.4.14). To lighten the notation we will now write $\nabla^{\mathrm{DS}}=\nabla_{U_{p}^{12}, \pi_{0, p}^{*} \sigma_{U^{2}}, U_{p+1}^{12}, \xi_{p+1}^{2}}^{\mathrm{DS}}$. By induction one has

$$
\begin{aligned}
\theta_{p+1} \nabla^{\mathrm{DS}}\left(\nabla_{\mathfrak{U}^{1}}^{p}\left(s_{U^{1}}\right)\right) & =\theta_{p+1} \nabla^{\mathrm{DS}}\left(\theta_{p} \cdots \theta_{1} g \nabla_{\mathfrak{U}^{2}}^{p}\left(s_{U^{2}}\right)+\sum_{j=1}^{p} P_{j}^{p} \pi_{j, p}^{*} \nabla_{\mathfrak{U}^{2}}^{j}\left(s_{U^{2}}\right)\right) \\
& =\theta_{p+1} \nabla^{\mathrm{DS}}\left(\theta_{p} \cdots \theta_{1} g \nabla_{\mathfrak{U}^{2}}^{p}\left(s_{U^{2}}\right)\right)+\sum_{j=1}^{p} \theta_{p+1} \nabla^{\mathrm{DS}}\left(P_{j}^{p} \nabla_{\mathfrak{U}^{2}}^{j}\left(s_{U^{2}}\right)\right) .
\end{aligned}
$$

Before continuing, observe that for any $f_{1}, f_{2} \in \mathscr{O}\left(U_{p}^{12}\right)$, one has (by an immediate computation)

$$
\nabla^{\mathrm{DS}}\left(f_{1} f_{2}\right)=f_{1} \nabla^{\mathrm{DS}}\left(f_{2}\right)+f_{2} d f_{1}\left(\xi_{p+1}^{2}\right) .
$$

Applying this to $f_{1}=\theta_{p} \cdots \theta_{1} g$ and $f_{2}=\nabla_{\mathfrak{U}^{2}}^{p}\left(s_{U^{2}}\right)$ we obtain

$$
\begin{aligned}
\theta_{p+1} \nabla^{\mathrm{DS}}\left(\theta_{p} \cdots \theta_{1} g \nabla_{\mathfrak{U}^{2}}^{p}\left(s_{U^{2}}\right)\right) & =\theta_{p+1} \theta_{p} \cdots \theta_{1} g \nabla^{\mathrm{DS}}\left(\nabla_{\mathfrak{U}^{2}}^{p}\left(s_{U^{2}}\right)\right)+\nabla_{\mathfrak{U}^{2}}^{p}\left(s_{U^{2}}\right) d\left(\theta_{p} \cdots \theta_{1} g\right)\left(\xi_{p+1}^{2}\right) \\
& =\theta_{p+1} \theta_{p} \cdots \theta_{1} g \nabla_{\mathfrak{U}^{2}}^{p+1}\left(s_{U^{2}}\right)+\nabla_{\mathfrak{U}^{2}}^{p}\left(s_{U^{2}}\right) d\left(\theta_{p} \cdots \theta_{1} g\right)\left(\xi_{p+1}^{2}\right) .
\end{aligned}
$$

Observe that the term $\nabla_{\mathfrak{U}^{2}}^{p}\left(s_{U^{2}}\right) d\left(\theta_{p} \cdots \theta_{1} g\right)\left(\xi_{p+1}^{2}\right)$ is of the form allowed in the last term of formula (2.4.14) at rank $p+1$. Therefore, the proof of formula (2.4.14) will be completed if one 
proves that for each $j<p$ the term $\theta_{p+1} \nabla^{\mathrm{DS}}\left(P_{j}^{p} \nabla_{\mathfrak{U}^{2}}^{j}\left(s_{U^{2}}\right)\right)$ is also of the form of an error term if (2.4.14) at rank $p+1$. To see this, observe that for each $j<p$ one has

$$
\begin{aligned}
\theta_{p+1} \nabla^{\mathrm{DS}}\left(P_{j}^{p} \nabla_{\mathfrak{U}^{2}}^{j}\left(s_{U^{2}}\right)\right) & =P_{j}^{p} \theta_{p+1} \nabla^{\mathrm{DS}}\left(\nabla_{\mathfrak{U}^{2}}^{j}\left(s_{U^{2}}\right)\right)+\nabla_{\mathfrak{U}^{2}}^{j}\left(s_{U^{2}}\right) d P_{j}^{p}\left(\xi_{p+1}^{2}\right) \\
& =P_{j}^{p} \theta_{p+1} \beta_{j, p} \nabla_{\mathfrak{U}^{2}}^{j+1}\left(s_{U^{2}}\right)+\nabla_{\mathfrak{U}^{2}}^{j}\left(s_{U^{2}}\right) d P_{j}^{p}\left(\xi_{p+1}^{2}\right),
\end{aligned}
$$

where $\beta_{j, p}$ is the function appearing in Lemma 2.4 applied to the trivialization tower $\mathfrak{U}^{2}$. This concludes the proof of (2.4.14).

To conclude the proof of the proposition, it remains to prove that $\omega_{D}^{\prime}\left(s_{1}, \ldots, s_{k}\right)$ is sent to $W_{D}\left(s_{1}, \ldots, s_{k}\right)$ under the above natural map. By continuity, it suffices to prove this over the open subset $X_{k}(D)^{\mathrm{reg}} \cap \pi_{k}^{-1}(X \backslash D)$. Take $w_{k} \in X_{k}(D)^{\text {reg }} \cap \pi_{k}^{-1}(X \backslash D)$. From the previous part of the proposition, we are allowed to choose any trivialization tower in order to make the computation of $\omega_{D}^{\prime}\left(s_{1}, \ldots, s_{k}\right)$ in a neighborhood of $w_{k}$. On the other hand, to compute the element $\omega_{D}\left(s_{1}, \ldots, s_{k}\right) \in H^{0}\left(X_{k}(D), \mathscr{O}_{X_{k}(D)}\left(k^{\prime}\right) \otimes \pi_{0, k}^{*} L\right)$ associated to $W_{D}\left(s_{1}, \ldots, s_{k}\right)$ under the isomorphism (1.4.13), we are allowed to use the explicit description isomorphism (1.3.9). Indeed, outside $D$ the logarithmic and absolute jet towers coincide. Let us therefore apply Theorem 1.2.(i).

Let $U \subset X \backslash D$ be an open set with local coordinates $\left(z_{1}, \ldots, z_{n}\right)$. Take a trivialization of $L_{\uparrow U}$ such that $\sigma_{D}$ is identically equal to 1 . It follows from [Dem97, Proof of Theorem 6.8] that $X_{k}(D)^{\text {reg }} \bigcap \pi_{0, k}^{-1}(U)$ can be covered by open sets $U \times \mathbb{C}^{(n-1) k}$. Indeed, consider the family of holomorphic curves

$$
\begin{aligned}
\gamma: U \times \mathbb{C}^{(n-1) k} \times \mathbb{C} & \rightarrow U \\
(z, w, t) & \mapsto \gamma_{(w, z)}(t)
\end{aligned}
$$

defined by

$$
\gamma_{(w, z)}^{i}(t)= \begin{cases}z_{i}+\frac{w_{i}^{(1)}}{1 !} t+\frac{w_{i}^{(2)}}{2 !} t^{2}+\cdots+\frac{w_{i}^{(k)}}{k !} t^{k} & \text { if } 1 \leqslant i \leqslant n-1 \\ z_{n}+t & \text { if } i=n\end{cases}
$$

where $w:=\left(w_{i}^{(j)}\right)_{\substack{1 \leqslant j \leqslant k \\ 1 \leqslant i \leqslant n}}$ and $\gamma_{(w, z)}:=\left(\gamma_{(w, z)}^{1}, \ldots, \gamma_{(w, z)}^{n}\right)$. To be precise, the map $\gamma$ is only defined on a open neighborhood of $U \times \mathbb{C}^{(n-1) k} \times\{0\}$ in $U \times \mathbb{C}^{(n-1) k} \times \mathbb{C}$, but this subtlety will be irrelevant as we will only consider the $k$-jets of each $\gamma_{(w, z)}$ at the point 0 .

In this setting, we will prove that its $k$-th lift $\left(\gamma_{(w, z)}\right)_{[k]}(0)$ gives a holomorphic embedding

$$
\tau_{k}: U \times \mathbb{C}^{(n-1) k} \rightarrow X_{k}(D)^{\mathrm{reg}}
$$

whose image is an open subset.

Let us take a special trivialization tower of order $k$, denoted by $\mathfrak{U}=\left(\left(U_{0}, \sigma_{U}\right),\left(U_{j}, \xi_{j}\right)_{1 \leqslant j \leqslant k}\right)$ in the following way:

(i) $\left(U_{0}, \sigma_{U}\right)=(U, 1)$.

(ii) Set $U_{1}=\left\{\left(\left[z_{1}^{(1)} \frac{\partial}{\partial z_{1}}+\cdots+z_{n-1}^{(1)} \frac{\partial}{\partial z_{n-1}}+\frac{\partial}{\partial z_{n}}\right] ; z\right) \in \mathrm{P}\left(T_{U}\right)\right\} \simeq \mathbb{C}^{n-1} \times U$ with the coordinate $\left(z ; z_{1}^{(1)}, \ldots, z_{n-1}^{(1)}\right)$. Define $\xi_{1}=z_{1}^{(1)} \frac{\partial}{\partial z_{1}}+\cdots+z_{n-1}^{(1)} \frac{\partial}{\partial z_{n-1}}+\frac{\partial}{\partial z_{n}} \in \Gamma\left(U_{1}, \mathscr{O}_{X_{1}(D)}(-1)\right)$, and take the basis for $V_{1 \uparrow U_{1}}$ as

$$
e_{1}^{(1)}=\frac{\partial}{\partial z_{1}^{(1)}}, \ldots, \quad e_{n-1}^{(1)}=\frac{\partial}{\partial z_{n-1}^{(1)}}, \quad e_{n}^{(1)}=\frac{\partial}{\partial z_{n}}+z_{1}^{(1)} \frac{\partial}{\partial z_{1}}+\cdots+z_{n-1}^{(1)} \frac{\partial}{\partial z_{n-1}} .
$$

Then one has $\left(\pi_{0,1}\right)_{*}\left(e_{n}^{(1)}\right)=\xi_{1}$, and $\pi_{0,1}\left(z ; z_{1}^{(1)}, \ldots, z_{n-1}^{(1)}\right)=z$.

(iii) Set $U_{2}=\left\{\left(\left[z_{1}^{(2)} e_{1}^{(1)}+\cdots+z_{n-1}^{(2)} e_{n-1}^{(1)}+e_{n}^{(1)}\right]\right) \in \mathrm{P}\left(V_{1}\right)_{\uparrow U_{1}}\right\} \simeq U \times \mathbb{C}^{2(n-1)}$ with the coordinate $\left(z ; z^{(1)}, z_{1}^{(2)}, \ldots, z_{n-1}^{(2)}\right)$. Here we write $z^{(1)}=\left(z_{1}^{(1)}, \ldots, z_{n-1}^{(1)}\right)$ for short. Define $\xi_{2}=z_{1}^{(2)} e_{1}^{(1)}+$ 
$\cdots+z_{n-1}^{(2)} e_{n-1}^{(1)}+e_{n}^{(1)} \in \Gamma\left(U_{2}, \mathscr{O}_{X_{2}}(-1)\right)$, and take the basis for $V_{2 \uparrow U_{2}}$ as

$$
e_{1}^{(2)}=\frac{\partial}{\partial z_{1}^{(2)}}, \ldots, \quad e_{n-1}^{(2)}=\frac{\partial}{\partial z_{n-1}^{(2)}}, \quad e_{n}^{(2)}=\frac{\partial}{\partial z_{n}}+z_{1}^{(1)} \frac{\partial}{\partial z_{1}}+\cdots+z_{n-1}^{(1)} \frac{\partial}{\partial z_{n-1}}+z_{1}^{(2)} \frac{\partial}{\partial z_{1}^{(1)}}+\cdots+z_{n-1}^{(2)} \frac{\partial}{\partial z_{n-1}^{(1)}}
$$

Then one has $\left(\pi_{1,2}\right)_{*}\left(e_{n}^{(2)}\right)=\xi_{2}$, and $\pi_{1,2}\left(z ; z^{(1)}, z_{1}^{(2)}, \ldots, z_{n-1}^{(2)}\right)=\left(z ; z^{(1)}\right)$.

(iv) Inductively, one can define $U_{p}$ with coordinates $\left(z ; z^{(1)}, \ldots, z^{(p)}\right) \in U \times \mathbb{C}^{(n-1) p}$ such that for any $j<p, \pi_{j, p}\left(z ; z^{(1)}, \ldots, z^{(p)}\right)=\left(z ; z^{(1)}, \ldots, z^{(j)}\right)$ and

$$
\xi_{p}=\frac{\partial}{\partial z_{n}}+z_{1}^{(1)} \frac{\partial}{\partial z_{1}}+\cdots+z_{n-1}^{(1)} \frac{\partial}{\partial z_{n-1}}+z_{1}^{(2)} \frac{\partial}{\partial z_{1}^{(1)}}+\cdots+z_{n-1}^{(2)} \frac{\partial}{\partial z_{n-1}^{(1)}}+\cdots+z_{1}^{(p)} \frac{\partial}{\partial z_{1}^{(p-1)}}+\cdots+z_{n-1}^{(p)} \frac{\partial}{\partial z_{n-1}^{(p-1)}}
$$

Then $\left(\pi_{j, p}\right)_{*}\left(\xi_{p}\right)=\xi_{j}$. In particular, by (1.4.11) and (1.4.12), for any $1 \leqslant j<p$, there is an isomorphism

$$
\mathscr{O}_{X_{p}(D)}(-1)_{\uparrow U_{p}} \underset{\xi_{p}}{\simeq} \underset{\mathscr{O}_{U_{p}}}{\simeq} \underset{\cdot\left(\pi_{j, p}\right)^{*} \xi_{j}}{\simeq} \underset{\left(\pi_{j, p}\right)^{*}\left(\pi_{j-1, p-1}\right)_{*}}{\simeq}\left(\pi_{j, p}\right)^{*} \mathscr{O}_{X_{j}(D)}(-1)_{\uparrow U_{p}}
$$

and

$$
\xi_{j}^{-1} \cdot\left(\pi_{j}\right)^{*} \xi_{j-1}=\xi_{j}^{-1} \cdot\left(\pi_{j}\right)^{*}\left(\pi_{j-1}\right)_{*}\left(\xi_{j}\right)=\Gamma_{j \mid U_{j}} .
$$

In this setting, one can prove that, within the coordinates for $U_{k}$, the $k$-th lift $\left(\gamma_{(w, z)}\right)_{[k]}(0)=$ $(z ; w)$. Hence $\tau_{k}: \mathbb{C}^{(n-1) k} \times U \rightarrow X_{k}(D)^{\text {reg }}$ whose image is the open subset $U_{k}$, and under the trivialization tower of order $k, \tau_{k}$ is an identity map. Moreover, $\left(\gamma_{(w, z)}\right)_{[k-1]}^{\prime}(0)=\xi_{k \uparrow\left(\gamma_{(w, z)}\right)_{[k]}(0)}$. Hence a straightforward computation shows that if we identify the parameter space $\mathbb{C}^{(n-1) k} \times U$ of $\gamma_{(w, z)}(t)$ with $U_{k}$ by

$$
\begin{aligned}
\tau_{k}: \mathbb{C}^{(n-1) k} \times U & \rightarrow U_{k} \\
\left(w^{(1)}, \ldots, w^{(k)} ; z\right) & \mapsto\left(\gamma_{(w, z)}\right)_{[k]}(0),
\end{aligned}
$$

then for any $f \in \Gamma\left(U, \mathscr{O}_{U}\right)$ and for any $j=1, \ldots, k$ one has

$$
d^{j}(f)\left(j_{k} \gamma_{(w, z)}\right)=\nabla_{\mathfrak{U}}^{j}(f)(w, z) \in \mathscr{O}\left(U_{j}\right) .
$$

For any $s_{1}, \ldots, s_{k} \in \Gamma\left(U, L_{\uparrow U}\right)$. Write $s_{1, U}, \ldots, s_{k, U} \in \mathscr{O}(U)$ for the local representatives of $s_{1}, \ldots, s_{k}$ under our choice of trivialization for $L_{\uparrow U}$ (i.e. $s_{i, U}=\frac{s_{i}}{\sigma_{D}}$ ). Then by (1.3.10), $\omega_{D}\left(s_{1, U}, \ldots, s_{k, U}\right) \in \Gamma\left(U_{k}, \mathscr{O}_{X_{k}(D)}\left(k^{\prime}\right)\right)$ is defined by

$$
\begin{aligned}
\omega_{D}\left(s_{1}, \ldots, s_{k}\right)(w, z) & =W_{D}\left(s_{1, U}, \ldots, s_{k, U}\right)\left(j_{k} \gamma_{(w, z)}\right) \cdot\left(\left(\gamma_{(w, z)}\right)_{[k-1]}^{\prime}(0)\right)^{-k^{\prime}} \\
& =\left|\begin{array}{ccc}
\nabla_{D}^{1}\left(s_{1, U}\right) & \cdots & \nabla_{D}^{1}\left(s_{k, U}\right) \\
\vdots & \ddots & \vdots \\
\nabla_{D}^{k}\left(s_{1, U}\right) & \cdots & \nabla_{D}^{k}\left(s_{k, U}\right)
\end{array}\right|\left(j_{k} \gamma_{(w, z)}\right) \cdot\left(\xi_{k}\right)^{-k^{\prime}} \\
& =\left|\begin{array}{ccc}
d^{1}\left(s_{1, U}\right) & \cdots & d^{1}\left(s_{k, U}\right) \\
\vdots & \ddots & \vdots \\
d^{k}\left(s_{1, U}\right) & \cdots & d^{k}\left(s_{k, U}\right)
\end{array}\right|\left(j_{k} \gamma_{(w, z)}\right) \cdot\left(\xi_{k}\right)^{-k^{\prime}}
\end{aligned}
$$

where the last equality is due to $\sigma_{U}=1$. Note that $d^{j}: \Gamma\left(U, \mathscr{O}_{U}\right) \rightarrow E_{j, j}^{\mathrm{GG}} \Omega_{U}$. Write

$$
\Gamma_{k}=k \pi_{2, k}^{*} \Gamma_{2}+(k+k-1) \pi_{3, k}^{*} \Gamma_{3}+\cdots+(k+\cdots+3) \pi_{k-1, k}^{*} \Gamma_{k-1}+(k+\cdots+2) \Gamma_{k}
$$


for short. Hence

$$
\begin{aligned}
\omega_{D}\left(s_{1}, \ldots, s_{k}\right)_{\uparrow U_{k}} & =\left|\begin{array}{ccc}
\nabla_{\mathfrak{U}}^{1}\left(s_{1, U}\right) & \cdots & \nabla_{\mathfrak{U}}^{1}\left(s_{k, U}\right) \\
\vdots & \ddots & \vdots \\
\nabla_{\mathfrak{U}}^{k}\left(s_{1, U}\right) & \cdots & \nabla_{\mathfrak{U}}^{k}\left(s_{k, U}\right)
\end{array}\right| \cdot\left(\xi_{k}\right)^{-k^{\prime}} \\
& =\omega_{\mathfrak{U}}\left(s_{1}, \ldots s_{k}\right) \cdot\left(\xi_{k}\right)^{-k^{\prime}} \\
& \stackrel{(2.4 .16)}{=} \omega_{\mathfrak{U}}\left(s_{1}, \ldots s_{k}\right) \cdot\left(\pi_{1, k}\right)^{*} \xi_{1}^{-k} \cdot\left(\pi_{2, k}\right)^{*} \xi_{2}^{-(k-1)} \cdots\left(\pi_{k-1, k}\right)^{*} \xi_{k-1}^{-2} \cdot \xi_{k}^{-1} \cdot \Gamma_{k} \\
& \stackrel{(2.4 .12)}{=} \omega_{D}^{\prime}\left(s_{1}, \ldots, s_{k}\right)_{\uparrow U_{k}} \cdot \Gamma_{k} .
\end{aligned}
$$

Since $U_{k}$ is dense in $\pi_{0, k}^{-1}(U)$ and since $\omega_{D}\left(s_{1}, \ldots, s_{k}\right)$ and $\omega_{D}^{\prime}\left(s_{1}, \ldots, s_{k}\right) \cdot \boldsymbol{\Gamma}_{k}$ coincide on $U_{k}$, it follows by continuity that they also coincide on $\pi_{0, k}^{-1}(U)$. Therefore it follows that these two sections coincide on $\pi_{0, k}^{-1}(X \backslash D)$ and therefore they also coincide on the whole space $X_{k}(D)$ :

$$
\omega_{D}\left(s_{1}, \ldots, s_{k}\right)=\omega_{D}^{\prime}\left(s_{1}, \ldots, s_{k}\right) \cdot \Gamma_{k} .
$$

The proposition is thus proved.

2.5. Logarithmic Wronskian ideal sheaf. Recall that in (2.3.7), we defined the $\log$ Wronskian morphism

$$
j^{k} W_{D}: \bigwedge^{k} J^{k} L \rightarrow E_{k, k^{\prime}} \Omega_{X}(\log D) \otimes L^{k}
$$

which is a morphism of $\mathscr{O}_{X}$-module. We denote by $\mathscr{W}_{k, L}:=j^{k} W_{D}\left(\bigwedge^{k} J^{k} L\right)$, which is a subsheaf of $E_{k, k^{\prime}} \Omega_{X}(\log D) \otimes L^{k}$. By (1.4.13) for any $m \in \mathbb{N}$ there exists a natural morphism

$$
\pi_{0, k}^{-1}\left(E_{k, k^{\prime}} \Omega_{X}(\log D) \otimes L^{m}\right) \simeq \pi_{0, k}^{-1}\left(\pi_{0, k}\right)_{*}\left(\mathscr{O}_{X_{k}(D)}\left(k^{\prime}\right) \otimes \pi_{0, k}^{*} L^{m}\right) \rightarrow \mathscr{O}_{X_{k}(D)}\left(k^{\prime}\right) \otimes \pi_{0, k}^{*} L^{m} .
$$

We denote by $\mathfrak{w}_{X_{k}(D)}$ the image of the composition

$$
\pi_{0, k}^{-1} \mathscr{W}_{k, L} \otimes \mathscr{O}_{X_{k}(D)}\left(-k^{\prime}\right) \otimes \pi_{0, k}^{*} L^{-k} \hookrightarrow \pi_{0, k}^{-1} E_{k, k^{\prime}} \Omega_{X}(\log D) \otimes \mathscr{O}_{X_{k}(D)}\left(-k^{\prime}\right) \rightarrow \mathscr{O}_{X_{k}(D)}
$$

which is a coherent ideal sheaf on $X_{k}(D) \cdot \mathfrak{w}_{X_{k}(D)}$ will be called the $k$-th logarithmic Wronskian ideal sheaf associated to the $\log$ manifold $(X, D)$. Let us denote by

$$
\mathbb{W}_{X_{k}(D)}:=\operatorname{Span}\left\{\omega_{D}\left(s_{1}, \ldots, s_{k}\right) \mid s_{1}, \ldots, s_{k} \in H^{0}(X, L)\right\}
$$

the sub-linear system of $\left|\mathscr{O}_{X_{k}(D)}\left(k^{\prime}\right) \otimes \pi_{0, k}^{*} L^{k}\right|$. One thus has the following result

Proposition 2.5. When $L$ generates $k$-jets everywhere on $X, \mathfrak{w}_{X_{k}(D)}$ is the base ideal of $\mathbb{W}_{X_{k}(D)}$. It satisfies moreover

$$
\operatorname{Supp}\left(\mathscr{O}_{X_{k}(D)} / \mathfrak{w}_{X_{k}(D)}\right) \subset X_{k}(D)^{\operatorname{sing}} \bigcup \pi_{0, k}^{-1}(D) .
$$

Proof. For any $s_{1}, \ldots, s_{k} \in H^{0}(X, L)$, let us define the natural linear map

$$
\begin{aligned}
& \bigwedge^{k} H^{0}(X, L) \rightarrow H^{0}\left(X, \bigwedge^{k} J^{k} L\right) \\
& s_{1} \wedge \cdots \wedge s_{k} \mapsto j_{L}^{k} s_{1} \wedge \cdots \wedge j_{L}^{k} s_{k} .
\end{aligned}
$$

It follows from the definition that

$$
W_{D}\left(s_{1}, \ldots, s_{k}\right)=j^{k} W_{D}\left(j_{L}^{k} s_{1} \wedge \cdots \wedge j_{L}^{k} s_{k}\right) \in H^{0}\left(X, \mathscr{W}_{k, L}\right) .
$$

Hence by the definition of $\mathfrak{w}_{X_{k}(D)}$ and the fact $\left(\pi_{0, k}\right)_{*} \omega_{D}\left(s_{1}, \ldots, s_{k}\right)=W_{D}\left(s_{1}, \ldots, s_{k}\right)$, one concludes that

$$
\omega_{D}\left(s_{1}, \ldots, s_{k}\right) \in H^{0}\left(X_{k}(D), \mathscr{O}_{X_{k}(D)}\left(k^{\prime}\right) \otimes \pi_{0, k}^{*} L^{k} \otimes \mathfrak{w}_{X_{k}(D)}\right) .
$$

In other words, the base ideal of $\mathscr{W}_{k, L}$ belongs to $\mathfrak{w}_{X_{k}(D)}$. 
On the other hand, since $L$ separates $k$-jets everywhere on $X$, the set of global sections

$$
\operatorname{Span}\left\{j_{L}^{k} s_{1} \wedge \cdots \wedge j_{L}^{k} s_{k} \in H^{0}\left(X, \bigwedge^{k} J^{k} L\right) \mid s_{1}, \ldots, s_{k} \in H^{0}(X, L)\right\}
$$

thus generates the locally free sheaf $\bigwedge^{k} J^{k} L$ everywhere on $X$. Recall that $J^{k} W_{D}: \bigwedge^{k} J^{k} L \rightarrow$ $\mathscr{W}_{k, L}$ is a surjective morphism between sheaves of $\mathscr{O}_{X}$-modules. Therefore, the set of sections

$$
\operatorname{Span}\left\{j^{k} W_{D}\left(j_{L}^{k} s_{1} \wedge \cdots \wedge j_{L}^{k} s_{k}\right) \in H^{0}\left(X, \mathscr{W}_{k, L}\right) \mid s_{1}, \ldots, s_{k} \in H^{0}(X, L)\right\}
$$

generates the sheaf of $\mathscr{O}_{X}$-module $\mathscr{W}_{k, L}$, which implies that $\mathfrak{w}_{X_{k}(D)}$ belongs to the base ideal of $\mathbb{W}_{X_{k}(D)}$ by the definition of $\mathfrak{w}_{X_{k}(D)}$. In conclusion, the base ideal of $\mathbb{W}_{X_{k}(D)}$ is $\mathfrak{w}_{X_{k}(D)}$. The second assertion follows from [Bro17, Lemma 2.4].

Let us now give a more detailed local description of these objects. Let $\mathbb{D}^{n}$ be the (unit) polydisc, and denote by $E:=\left\{\left(z_{1}, \ldots, z_{n}\right) \in \mathbb{D}^{n} \mid z_{1}=0\right\}$. As in (2.3.7), we define a morphism of $\mathscr{O}_{\mathbb{D}^{n}}$-module associated to the log pair $\left(\mathbb{D}^{n}, E\right)$

$$
J^{k} W_{E}: \bigwedge^{k} J^{k} \mathscr{O}_{\mathbb{D}^{n}} \rightarrow E_{k, k^{\prime}} \Omega_{\mathbb{D}^{n}}(\log E)
$$

Set

$$
J^{k} W: \bigwedge^{k} J^{k} \mathscr{O}_{\mathbb{D}^{n}} \rightarrow E_{k, k^{\prime}} \Omega_{\mathbb{D}^{n}}
$$

to be the morphism of $\mathscr{O}_{\mathbb{D}^{n}}$-module induced by the following map

$$
\begin{aligned}
\bigwedge H^{0}\left(\mathbb{D}^{n}, \mathscr{O}_{\mathbb{D}^{n}}\right) & \rightarrow H^{0}\left(\mathbb{D}^{n}, E_{k, k^{\prime}} \Omega_{\mathbb{D}^{n}}\right) \\
f_{1} \wedge \cdots \wedge f_{k} & \mapsto\left|\begin{array}{ccc}
d^{1} f_{1} & \cdots & d^{1} f_{k} \\
\vdots & \ddots & \vdots \\
d^{k} f_{1} & \cdots & d^{k} f_{k}
\end{array}\right| .
\end{aligned}
$$

Fix an open covering $\mathfrak{U}$ of $X$ such that for any open set $U \in \mathfrak{U}, L_{\uparrow U}$ can be trivialized and such that one has the following dichotomy:

(1) $(U, D \cap U)$ is biholomorphic to $\left(\mathbb{D}^{n}, E\right)$, and under the trivialization of $L_{\uparrow U}, \sigma_{D}=z_{1}$.

(2) $U \cap D=\emptyset$, and under the trivialization of $L_{\uparrow U}, \sigma_{D}=1$.

It follows from the very definition that one has the following local trivialization of the morphism $J^{k} W_{D}$ in Case (1),

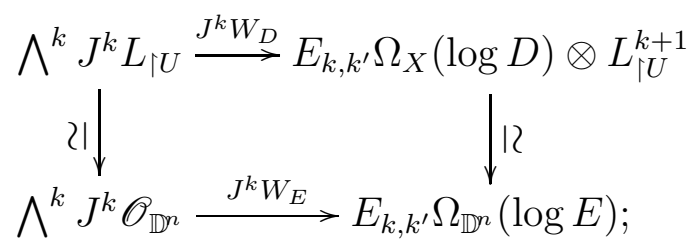

and in Case (2)

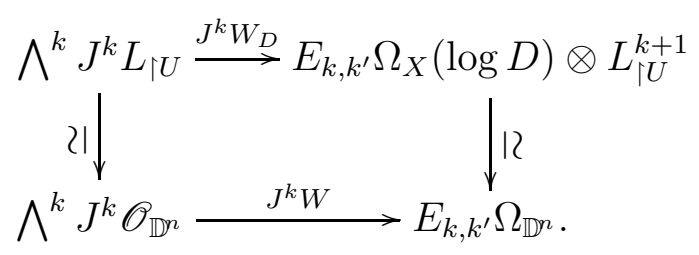

Therefore, we conclude that the local models of the logarithmic Wronskians are universal.

Let us denote by $\mathbb{D}_{k}^{n}$ (resp. $\left.\mathbb{D}_{k}^{n}(E)\right)$ the (resp. logarithmic) Demailly-Semple $k$-jet tower of $\left(\mathbb{D}^{n}, T_{\mathbb{D}^{n}}\right)$ (resp. $\left.\left(\mathbb{D}^{n}, E, T_{\mathbb{D}^{n}}(-\log E)\right)\right)$. By $(1.4 .13)$, there are natural morphisms of $\mathscr{O}_{\mathbb{B}_{k}^{n}}(E)^{-}$ module and $\mathscr{O}_{\mathbb{D}_{k}^{n}}$-module

$$
\pi_{0, k}^{-1} E_{k, k^{\prime}} \Omega_{\mathbb{D}^{n}}(\log E) \rightarrow \mathscr{O}_{\mathbb{D}_{k}^{n}(E)}\left(k^{\prime}\right), \quad \pi_{0, k}^{-1} E_{k, k^{\prime}} \Omega_{\mathbb{D}^{n}} \rightarrow \mathscr{O}_{\mathbb{D}_{k}^{n}}\left(k^{\prime}\right) .
$$


Set $\mathscr{W}_{k, E}$ and $\mathscr{W}_{k}$ to be the images of $J^{k} W_{E}$ and $J^{k} W$. Therefore,

$$
\begin{aligned}
\pi_{0, k}^{-1} \mathscr{W}_{k, E} \otimes \mathscr{O}_{\mathbb{D}_{k}^{n}(E)}\left(-k^{\prime}\right) & \hookrightarrow \pi_{0, k}^{-1} E_{k, k^{\prime}} \Omega_{\mathbb{D}^{n}}(\log E) \otimes \mathscr{O}_{\mathbb{D}_{k}^{n}(E)}\left(-k^{\prime}\right) \rightarrow \mathscr{O}_{\mathbb{D}_{k}^{n}(E)}, \\
\pi_{0, k}^{-1} \mathscr{W}_{k} \otimes \mathscr{O}_{\mathbb{D}_{k}^{n}}\left(-k^{\prime}\right) & \hookrightarrow \pi_{0, k}^{-1} E_{k, k^{\prime}} \Omega_{\mathbb{D}^{n}} \otimes \mathscr{O}_{\mathbb{D}_{k}^{n}}\left(-k^{\prime}\right) \rightarrow \mathscr{O}_{\mathbb{D}_{k}^{n}},
\end{aligned}
$$

whose images are coherent ideal sheaves, which we denote by $\mathfrak{w}_{\mathbb{D}_{k}^{n}(E)} \subset \mathscr{O}_{\mathbb{D}_{k}^{n}(E)}$ and $\mathfrak{w}_{\mathbb{D}_{k}^{n}} \subset \mathscr{O}_{\mathbb{D}_{k}^{n}}$. Then for any $U \in \mathfrak{U}$, under the trivialization $X_{k}(D)_{\uparrow U} \simeq \mathbb{D}_{k}^{n}(E)$ in Case $(1)^{k}$ and $X_{k}(D)_{\uparrow U} \simeq \mathbb{D}_{k}^{n}$ in Case (2), one has the isomorphisms $\mathfrak{w}_{X_{k}(D) \mid U} \simeq \mathfrak{w}_{\mathbb{D}_{k}^{n}(E)}$ and $\mathfrak{w}_{X_{k}(D) \mid U} \simeq \mathfrak{w}_{\mathbb{D}_{k}^{n}}$ respectively. This local description will be used to establish a certain universal property in $\S 2.7$.

2.6. Universal property of logarithmic Wronskian ideal sheaves. Let us begin with the following setting. Let $A$ be a very ample line bundle over a smooth projective manifold $Y$, and let $\mathbb{L}$ be the total space of the line bundle $A^{m}$ for some $m \in \mathbb{N}^{*}$. Denote by $p: \mathbb{L} \rightarrow Y$ the natural projection map with $L:=p^{*} A^{m}$, and $T \in H^{0}(\mathbb{L}, L)$ the tautological section such that $T(x)=x$ for any $x \in \mathbb{L}$. Note that $Y$ can be seen as the smooth hypersurface of $\mathbb{L}$ defined by $\{x \in \mathbb{L} \mid T(x)=0\}$. Then according to $\S 2.2$, there exists for any $k \in \mathbb{N}$ a natural higher order logarithmic connection $\nabla^{k}: \mathscr{O}_{\mathbb{L}}(L) \rightarrow \mathscr{O}_{\mathbb{L}}(L) \otimes E_{k, k}^{\mathrm{GG}} \Omega_{\mathbb{L}}(\log Y)$ associated to the $\log$ manifold $(\mathbb{L}, Y)$. For any sections $s_{1}, \ldots, s_{k} \in H^{0}\left(Y, A^{m}\right)$, it follows from (2.3.6) that one has the associated logarithmic Wronskian

$$
W_{\mathbb{L}, Y}\left(p^{*} s_{1}, \ldots, p^{*} s_{k}\right) \in H^{0}\left(\mathbb{L}, E_{k, k^{\prime}} \Omega_{\mathbb{L}}(\log Y) \otimes L^{k}\right) .
$$

By (1.4.13), there exists a unique section in $H^{0}\left(\mathbb{L}_{k}, \mathscr{O}_{\mathbb{L}_{k}}\left(k^{\prime}\right) \otimes \pi_{0, k}^{*} L^{k}\right)$, denoted by $\omega_{\log }\left(s_{1}, \ldots, s_{k}\right)$, such that

$$
\left(\pi_{0, k}\right)_{*} \omega_{\log }\left(s_{1}, \ldots, s_{k}\right)=W_{\mathbb{L}, Y}\left(p^{*} s_{1}, \ldots, p^{*} s_{k}\right)
$$

where $\mathbb{L}_{k}$ denotes to be the $\log$ Demailly $k$-jet tower of $\left(\mathbb{L}, Y, T_{\mathbb{L}}(-\log Y)\right)$.

Let us denote by $\omega_{\log }^{\prime}\left(s_{1}, \ldots, s_{k}\right)$ the logarithmic Wronskian defined in Proposition 2.3. Then

$$
\omega_{\log }\left(s_{1}, \ldots, s_{k}\right)=\omega_{\log }^{\prime}\left(s_{1}, \ldots, s_{k}\right) \cdot \Gamma_{k},
$$

where $\boldsymbol{\Gamma}_{k}$ is an effective divisor of $\mathbb{L}_{k}$ defined in (2.4.17). Consider the linear systems

$$
\begin{aligned}
\mathbb{W}_{k, \mathbb{L}, Y} & :=\operatorname{Span}\left\{\omega_{\log }\left(s_{1}, \ldots, s_{k}\right) \mid s_{1}, \ldots, s_{k} \in H^{0}\left(Y, A^{m}\right)\right\} \\
\mathbb{W}_{k, \mathbb{L}, Y}^{\prime} & :=\operatorname{Span}\left\{\omega_{\log }^{\prime}\left(s_{1}, \ldots, s_{k}\right) \mid s_{1}, \ldots, s_{k} \in H^{0}\left(Y, A^{m}\right)\right\}
\end{aligned}
$$

and define $\mathfrak{w}_{k, \mathbb{L}, Y}$ and $\mathfrak{w}_{k, \mathbb{L}, Y}^{\prime}$ to be their base ideal. By (2.6.19), one has

$$
\mathfrak{w}_{k, \mathbb{L}, Y}=\mathfrak{w}_{k, \mathbb{L}, Y}^{\prime} \cdot \mathscr{O}_{\mathbb{L}_{k}}\left(-\boldsymbol{\Gamma}_{k}\right) .
$$

It follows from the definition of $W_{\mathbb{L}, Y}$ that, there exists a morphism of $\mathscr{O}_{\mathbb{L}}$-module

$$
j^{k} W_{\mathbb{L}, Y}: \bigwedge^{k} p^{*}\left(J^{k} A^{m}\right) \rightarrow E_{k \cdot k^{\prime}} \Omega_{\mathbb{L}}(\log Y) \otimes L^{k}
$$

such that $W_{\mathbb{L}, Y}$ factors through this morphism. Set $\mathscr{W}_{k, \mathbb{L}, Y}$ to be the image of $j^{k} W_{\mathbb{L}, Y}$. We will study the properties of $j^{k} W_{\mathbb{L}, Y}$ locally.

Take an open set $U$ with coordinates $\left(z_{1}, \ldots, z_{n}\right)$ such that $A_{\uparrow U}$ can be trivialized. Then there are local coordinates $\left(t, z_{1}, \ldots, z_{n}\right)$ for $p^{-1}(U) \simeq U \times \mathbb{C}$, such that $L_{\left\lceil p^{-1}(U)\right.}$ is trivialized with $T_{\left\lceil p^{-1}(U)\right.}=t$. Hence the divisor $Y \cap p^{-1}(U)$ is defined by the local equation $(t=0)$. One thus can regard $U$ as a smooth divisor in $p^{-1}(U)$ defined by $(t=0)$. For any $k \in \mathbb{N}$, write $\nabla_{U}^{k}: \mathscr{O}_{U \times \mathbb{C}} \rightarrow E_{k, k}^{\mathrm{GG}} \Omega_{U \times \mathbb{C}}(\log U)$ for the higher order logarithmic connection defined in (2.2.3). In view of (2.3.6) we define

$$
\begin{aligned}
W_{p^{-1}(U), U}: \bigwedge \mathscr{O}(U) & \rightarrow \Gamma\left(U \times \mathbb{C}, E_{k, k^{\prime}} \Omega_{U \times \mathbb{C}}(\log U)\right) \\
f_{1} \wedge \cdots \wedge f_{k} & \mapsto\left|\begin{array}{ccc}
\nabla_{U}^{1}\left(p_{U}^{*} f_{1}\right) & \cdots & \nabla_{U}^{1}\left(p_{U}^{*} f_{k}\right) \\
\vdots & \ddots & \vdots \\
\nabla_{U}^{k}\left(p_{U}^{*} f_{1}\right) & \cdots & \nabla_{U}^{k}\left(p_{U}^{*} f_{k}\right)
\end{array}\right|
\end{aligned}
$$


where $p_{U}: U \times \mathbb{C} \rightarrow U$ is the natural projection map. By Lemma 2.2, one has

$$
W_{p^{-1}(U), U}\left(f_{1} \wedge \cdots \wedge f_{k}\right)=\left|\begin{array}{cccc}
1 & d^{0}\left(p_{U}^{*} f_{1}\right) & \cdots & d^{0}\left(p_{U}^{*} f_{k}\right) \\
\frac{1}{t} d^{1} t & d^{1}\left(p_{U}^{*} f_{1}\right) & \cdots & d^{1}\left(p_{U}^{*} f_{k}\right) \\
\vdots & \vdots & \ddots & \vdots \\
\frac{1}{t} d^{k} t & d^{k}\left(p_{U}^{*} f_{1}\right) & \cdots & d^{k}\left(p_{U}^{*} f_{k}\right)
\end{array}\right|
$$

Observe that $d^{i}\left(p_{U}^{*} f_{j}\right)$ does not contain any $d^{\ell} \log t$, therefore (2.6.22) induces a morphism between locally free sheaves of $\mathscr{O}_{p^{-1}(U)^{-} \text {modules }}$

$$
j^{k} W_{p^{-1}(U), U}: \bigwedge^{k} p^{*}\left(J^{k} \mathscr{O}_{U}\right) \rightarrow E_{k, k^{\prime}} \Omega_{U \times \mathbb{C}}(\log U)
$$

so that $W_{p^{-1}(U), U}$ factors through this morphism. If we use the basis for the local trivialization of $E_{k, k^{\prime}}^{G G} \Omega_{U \times \mathbb{C}}(\log U)$ in Lemma 1.1 and the standard basis for the trivialization of $J^{k} \mathscr{O}_{U}$ induced by the coordinates system $\left(z_{1}, \ldots, z_{n}\right)$, then by $(2.6 .23), j^{k} W_{p^{-1}(U), U}$ is represented by a constant matrix with respect to these trivializations. In particular, the image of $j^{k} W_{U}$, denoted by $\mathscr{W}_{k, U}$, is a locally free sheaf. In this setting, $j^{k} W_{p^{-1}(U), U}$ trivializes $j^{k} W_{\mathbb{L}, Y}$. Hence $\mathscr{W}_{k, \mathbb{L}, Y} \subset$ $E_{k, k^{\prime}} \Omega_{\mathbb{L}}(\log Y) \otimes L^{k}$ is a locally free sheaf of $\mathscr{O}_{\mathbb{L}}$-module on $\mathbb{L}$. As in Proposition 2.5, one has the following

Proposition 2.6. For $L:=p^{*} A^{m}$, when $m \geqslant k$, the ideal sheaf $\mathfrak{w}_{k, \mathbb{L}, Y}$ coincides with the image

$$
\varphi_{k}: \pi_{0, k}^{-1} \mathscr{W}_{k, \mathbb{L}, Y} \otimes \mathscr{O}_{\mathbb{L}_{k}}\left(-k^{\prime}\right) \otimes \pi_{0, k}^{*} L^{-k} \hookrightarrow \pi_{0, k}^{-1} E_{k, k^{\prime}} \Omega_{\mathbb{L}}(\log Y) \otimes \mathscr{O}_{\mathbb{L}_{k}}\left(-k^{\prime}\right) \rightarrow \mathscr{O}_{\mathbb{L}_{k}}
$$

where $\mathscr{O}_{\mathbb{L}_{k}}(1)$ denotes to be the tautological line bundle defined in $\S 1.4$.

Proof. For any $s_{1}, \ldots, s_{k} \in H^{0}\left(Y, A^{m}\right)$, one has the following natural linear map from the global sections to their $k$-jets

$$
\begin{aligned}
\bigwedge^{k} H^{0}\left(Y, A^{m}\right) & \rightarrow H^{0}\left(Y, \bigwedge^{k} J^{k} A^{m}\right) \\
s_{1} \wedge \cdots \wedge s_{k} & \mapsto j^{k} s_{1} \wedge \cdots \wedge j^{k} s_{k}
\end{aligned}
$$

Here we write $j^{k}$ instead of $j_{A^{m}}^{k}$ to lighten the notation. Recall that

$$
W_{\mathbb{L}, Y}\left(s_{1}, \ldots, s_{k}\right)=j^{k} W_{\mathbb{L}, Y}\left(p^{*} j^{k} s_{1} \wedge \cdots \wedge p^{*} j^{k} s_{k}\right) \in H^{0}\left(\mathbb{L}, \mathscr{W}_{k, \mathbb{L}, Y}\right),
$$

and thus by the definition of $\mathfrak{w}_{k, \mathbb{L}, Y}$ and the fact $\left(\pi_{0, k}\right)_{*} \omega_{\log }\left(s_{1}, \ldots, s_{k}\right)=W_{\mathbb{L}, Y}\left(s_{1}, \ldots, s_{k}\right)$, one has

$$
\mathfrak{w}_{k, \mathbb{L}, Y} \subseteq \operatorname{Im}\left(\varphi_{k}\right) .
$$

On the other hand, since $A$ is very ample and $m \geqslant k$, then $A^{m}$ generates $k$-jets, and the set

$$
\left.\operatorname{Span}\left\{p^{*} j^{k} s_{1} \wedge \cdots \wedge p^{*} j^{k} s_{k}\right) \in H^{0}\left(\mathbb{L}, \bigwedge^{k} p^{*} J^{k} A^{m}\right) \mid s_{1}, \ldots, s_{k} \in H^{0}\left(Y, A^{m}\right)\right\}
$$

generates the locally free sheaf of $\mathscr{O}_{\mathbb{L}}$-module $\bigwedge^{k} p^{*} J_{k} A^{m}$ everywhere on $\mathbb{L}$. It follows from the definition that $J^{k} W_{\mathbb{L}, Y}: \bigwedge^{k} p^{*} J^{k} A^{m} \rightarrow \mathscr{W}_{k, \mathbb{L}, Y}$ is a surjective morphism between sheaves of $\mathscr{O}_{\mathbb{L}}$-modules. Therefore, the set of sections

$$
\operatorname{Span}\left\{j^{k} W_{\mathbb{L}, Y}\left(p^{*} j^{k} s_{1} \wedge \cdots \wedge p^{*} j^{k} s_{k}\right) \in H^{0}\left(\mathbb{L}, \mathscr{W}_{k, \mathbb{L}, Y}\right) \mid s_{1}, \ldots, s_{k} \in H^{0}\left(Y, A^{m}\right)\right\}
$$

generates the sheaf of $\mathscr{O}_{\mathbb{L}}$-module $\mathscr{W}_{k, \mathbb{L}, Y}$, and one thus has $\mathfrak{w}_{k, \mathbb{L}, Y} \supseteq \operatorname{Im}\left(\varphi_{k}\right)$. This implies the result. 
2.7. Universal family of log Demailly towers of general log pairs and its blow-up. As we did in [BD17], the construction of the $\log$ pair $(\mathbb{L}, Y)$ enables us to "linearize" the family of $\log$ manifolds $(Y, D)$ with $D$ varying in the linear system $\left|A^{m}\right|$. Indeed, for any $\sigma \in H^{0}\left(Y, A^{m}\right)$, consider the hypersurface $H_{\sigma} \subset \mathbb{L}$ defined to be the zero locus of the section

$$
T-p^{*} \sigma \in H^{0}\left(\mathbb{L}, p^{*} A^{m}\right) .
$$

When the zero locus $D_{\sigma}$ of $\sigma$ is a smooth hypersurface on $Y, H_{\sigma}$ will also be smooth. A crucial observation is that

$$
p_{\sigma}=p_{\left\lceil H_{\sigma}\right.}:\left(H_{\sigma}, D_{\sigma}\right) \rightarrow\left(Y, D_{\sigma}\right)
$$

is a biholomorphism between $\log$ manifolds, and the hyperbolicity of $Y \backslash D_{\sigma}$ is therefore equivalent to that of $H_{\sigma} \backslash D_{\sigma}$. Moreover, we have the functoriality of the logarithmic Wronskians ideal sheaves.

Lemma 2.7. We denote by $H_{\sigma, k}$ the $\log$ Demailly $k$-jet tower of $\left(H_{\sigma}, D_{\sigma}, T_{H_{\sigma}}\left(-\log D_{\sigma}\right)\right)$, and let $\mathfrak{w}_{H_{\sigma, k}}$ be the $k$-th logarithmic Wronskian ideal sheaf of $H_{\sigma, k}$ defined in $\S 2.5$. When $m \geqslant k$, we have

$$
\mathfrak{w}_{k, \mathbb{L}, Y \nmid H_{\sigma, k}}=\mathfrak{w}_{H_{\sigma, k}} .
$$

Proof. Recall that

$$
p_{\sigma}=p_{\left\lceil H_{\sigma}\right.}:\left(H_{\sigma}, D_{\sigma}\right) \rightarrow\left(Y, D_{\sigma}\right)
$$

is a biholomorphism between $\log$ manifolds. Write $L_{\sigma}:=p_{\sigma}^{*} A^{m}=L_{\uparrow H_{\sigma}}$. Then $p_{\sigma}$ induces an isomorphism of linear spaces of global sections

$$
\begin{aligned}
H^{0}\left(Y, A^{m}\right) & \stackrel{\simeq}{\rightarrow} H^{0}\left(H_{\sigma}, L_{\sigma}\right) \\
s & \mapsto p_{\sigma}^{*} s .
\end{aligned}
$$

By the functoriality of the logarithmic Wronskians in (2.3.10), for any $s_{1}, \ldots, s_{k} \in H^{0}\left(Y, A^{m}\right)$, we have

$$
\omega_{\log }\left(s_{1}, \ldots, s_{k}\right)_{\left\lceil H_{\sigma, k}\right.}=\omega_{D_{\sigma}}\left(p_{\sigma}^{*} s_{1}, \ldots, p_{\sigma}^{*} s_{k}\right) \in H^{0}\left(H_{\sigma, k}, \mathscr{O}_{H_{\sigma, k}}\left(k^{\prime}\right) \otimes \pi_{0, k}^{*} L_{\sigma}^{k}\right) .
$$

Hence $\mathfrak{w}_{k, \mathbb{L}, Y \nmid H_{\sigma, k}}$ is the base ideal sheaf of the linear system

$$
\mathbb{W}_{H_{\sigma, k} ; L_{\sigma}}:=\operatorname{Span}\left\{\omega_{D_{\sigma}}\left(s_{1}^{\prime}, \ldots, s_{k}^{\prime}\right) \mid s_{1}^{\prime}, \ldots, s_{k}^{\prime} \in H^{0}\left(H_{\sigma}, L_{\sigma}\right)\right\} .
$$

We note that when $m \geqslant k, A^{m}$ generates $k$-jets everywhere on $Y$, therefore so does $L_{\sigma}$. The lemma then follows immediately from Proposition 2.5.

Now let us consider the universal family of hypersurfaces $\mathscr{H}^{\mathrm{u}} \subset \mathbb{L} \times H^{0}\left(Y, A^{m}\right)$ defined by

$$
\mathscr{H}^{\mathrm{u}}:=\left\{(x, \sigma) \in \mathbb{L} \times H^{0}\left(Y, A^{m}\right) \mid T(x)-p^{*} \sigma(x)=0\right\},
$$

and define the family of hypersurfaces in $Y$ by

$$
\mathscr{D}^{\mathrm{u}}:=\left\{(y, \sigma) \in Y \times H^{0}\left(Y, A^{m}\right) \mid \sigma(y)=0\right\} .
$$

One can take a non-empty Zariski open set $\mathbb{A}^{\mathrm{s}}$ of the parameter space $\mathbb{A}^{\mathrm{u}}:=H^{0}\left(Y, A^{m}\right)$ such that, the shrinking $\log$ family over $\mathbb{A}^{\mathrm{s}}$, denote by of $\left(\mathscr{H}^{\mathrm{s}}, \mathscr{D}^{\mathrm{s}}\right) \rightarrow \mathbb{A}^{\mathrm{s}}$, is smooth. Set $\mathscr{H}_{k}^{\mathrm{s}}$ to be the $\log$ Demailly $k$-jet tower of $\left(\mathscr{H}^{\mathrm{s}}, \mathscr{D}^{\mathrm{s}}, T_{\mathscr{H}^{\mathrm{s}} / \mathbb{A}^{\mathrm{s}}}\left(-\log \mathscr{D}^{\mathrm{s}}\right)\right)$, and denote by $q_{k}: \mathscr{H}_{k}^{\mathrm{s}} \rightarrow \mathbb{A}^{\mathrm{s}}$ the natural projection. By the choice of $\mathbb{A}^{\mathrm{s}}$, one notes that for any $\sigma \in \mathbb{A}^{\mathrm{s}}$, the fiber $q_{k}^{-1}(\sigma)$ is $H_{\sigma, k}$. Observe that we have an embedding $\mathscr{H}_{k}^{\mathrm{s}} \hookrightarrow \mathbb{L}_{k} \times \mathbb{A}^{\mathrm{s}}$. Let us denote by

$$
\mathfrak{w}_{\mathscr{H}_{k}^{\mathrm{s}}}:=\operatorname{pr}_{1}^{*}\left(\mathfrak{w}_{k, \mathbb{L}, Y}\right)_{\mid \mathscr{H}_{k}^{\mathrm{s}}},
$$

where $\operatorname{pr}_{1}: \mathbb{L}_{k} \times \mathbb{A}^{\mathrm{u}} \rightarrow \mathbb{L}_{k}$ is the natural projection map. By Lemma 2.7, we have

$$
\mathfrak{w}_{\mathscr{P}_{k}^{\mathrm{s}}\left\lceil H_{\sigma, k}\right.}=\mathfrak{w}_{H_{\sigma, k}} .
$$

In some sense, the ideal $\mathfrak{w}_{k, \mathbb{L}, Y}$ is the obstruction to the positivity of $\mathscr{O}_{\mathbb{L}_{k}}(1)$. Therefore, let us define $\nu_{k}: \widetilde{\mathbb{L}}_{k} \rightarrow \mathbb{L}_{k}$ to be the blow-up of the ideal sheaf $\mathfrak{w}_{k, \mathbb{L}, Y}$. It follows from (2.6.20) that 
$\nu_{k}$ is also the blow-up for $\mathfrak{w}_{k, \mathbb{L}, Y}^{\prime}$ (see [Har77, Chapter II, Exercise 7.11]). We denote by $F$ and $F^{\prime}$ the effective divisors in $\widetilde{\mathbb{L}}_{k}$ such that

$$
\nu_{k}^{*} \mathfrak{w}_{k, \mathbb{L}, Y}=\mathscr{O}_{\widetilde{\mathbb{L}}_{k}}(-F) \quad \text { and } \quad \nu_{k}^{*} \mathfrak{w}_{k, \mathbb{L}, Y}^{\prime}=\mathscr{O}_{\widetilde{\mathbb{L}}_{k}}\left(-F^{\prime}\right)
$$

We define $\mu_{k}: \widetilde{\mathscr{H}_{k}^{\mathrm{s}}} \rightarrow \mathscr{H}_{k}^{\mathrm{s}}$ to be the blow-up of the ideal sheaf $\mathfrak{w}_{\mathscr{H}_{k}^{\mathrm{s}}}$ with $\mathscr{O}_{\mathscr{\mathscr { H }}_{k}^{\mathrm{s}}}(-\tilde{F}):=\mu_{k}^{*} \mathfrak{w}_{\mathscr{H}_{k}^{\mathrm{s}}}$. By the universal property of the blow-up, one has the commutative diagram



The following lemma enables us to reduce the desired "general Kobayashi hyperbolicity" to a construction of a particular example satisfying a strong Zariski open property.

Lemma 2.8. When $\mu_{k}$ is restricted on each fiber $\widetilde{H}_{\sigma, k}$ of $\widetilde{\mathscr{H}_{k}^{\mathrm{s}}} \rightarrow \mathbb{A}^{\mathrm{s}}, \mu_{\sigma, k}=\mu_{k \uparrow \widetilde{H}_{\sigma, k}}: \widetilde{H}_{\sigma, k} \rightarrow H_{\sigma, k}$ is nothing but the blow-up of the ideal sheaf $\mathfrak{w}_{H_{\sigma, k}}$.

Proof. Let us first observe that as a consequence of the local inverse theorem in several complex variables we obtain that in the analytic category, families of smooth pairs $\left(\mathscr{H}^{\mathrm{s}}, \mathscr{D}^{\mathrm{s}}\right) \stackrel{\rho}{\rightarrow} \mathbb{A}^{\mathrm{s}}$ are locally trivial in the following sense: for any $x \in \mathscr{H}^{\mathrm{s}}$ there exists a neighborhood $\Omega \subset \mathscr{H}^{\mathrm{s}}$, a neighborhood $V \subset \mathbb{A}^{\mathrm{s}}$ of $\sigma:=\rho(x)$ and an open subset $U \subset \mathbb{C}^{n}$ with coordinates $\left(z_{1}, \ldots, z_{n}\right)$ such that there exists an isomorphism

$$
\Phi: U \times V \stackrel{\simeq}{\rightarrow} \Omega
$$

satisfying $\rho \circ \Phi=\operatorname{pr}_{2}$ (where $\operatorname{pr}_{2}: U \times V \rightarrow V$ is the projection on the first factor) and such that

$$
\Phi^{*} \mathscr{D}^{\mathrm{s}}=\left(z_{1}=0\right) \quad \text { or } \quad \mathscr{D}^{\mathrm{s}} \cap \Omega=\varnothing .
$$

By the local description of the logarithmic Wronskian ideal sheaves established in $\S 2.5$, via the isomorphism $\Phi$ one has

$$
\mathfrak{w}_{\mathscr{H}_{k}^{\mathrm{s}} \mid \pi_{0, k}^{-1}(\Omega)} \simeq \operatorname{pr}_{1}^{*} \mathfrak{w}_{\mathbb{D}_{k}^{n}(E)} \quad \text { or } \quad \mathfrak{w}_{\mathscr{H}_{k}^{\mathrm{s}} \mid \pi_{0, k}^{-1}(\Omega)} \simeq \operatorname{pr}_{1}^{*} \mathfrak{w}_{\mathbb{D}_{k}^{n}}
$$

This implies the result of the lemma.

On the other hand, for any sections $s_{1}, \ldots, s_{k} \in H^{0}\left(Y, A^{m}\right)$, by (2.6.20) and (2.7.24) there exists a (unique) section

$$
\begin{aligned}
\tilde{\omega}_{\log }\left(s_{1}, \ldots, s_{k}\right) & \in H^{0}\left(\widetilde{\mathbb{L}}_{k}, \nu_{k}^{*}\left(\mathscr{O}_{\mathbb{L}_{k}}\left(k^{\prime}\right) \otimes \pi_{0, k}^{*} L^{k}\right) \otimes \mathscr{O}_{\widetilde{\mathbb{L}}_{k}}(-F)\right), \\
& =H^{0}\left(\widetilde{\mathbb{L}}_{k}, \nu_{k}^{*}\left(\mathscr{O}_{\mathbb{L}_{k}}(k, k-1, \ldots, 1) \otimes \pi_{0, k}^{*} L^{k}\right) \otimes \mathscr{O}_{\widetilde{\mathbb{L}}_{k}}\left(-F^{\prime}\right)\right)
\end{aligned}
$$

such that

$$
\begin{aligned}
\nu_{k}^{*} \omega_{\log }\left(s_{1}, \ldots, s_{k}\right) & =\tilde{\omega}_{\log }\left(s_{1}, \ldots, s_{k}\right) \cdot F, \\
\nu_{k}^{*} \omega_{\log }^{\prime}\left(s_{1}, \ldots, s_{k}\right) & =\tilde{\omega}_{\log }\left(s_{1}, \ldots, s_{k}\right) \cdot F^{\prime} .
\end{aligned}
$$

We will also need the following crucial lemma.

Lemma 2.9. For any sections $s_{1}, \ldots, s_{k}, s_{1}^{\prime}, \ldots, s_{k}^{\prime} \in H^{0}\left(Y, A^{m}\right)$ and any point $y \in Y$, if the $k$ jets $j^{k} s_{i}(y)=j^{k} s_{i}^{\prime}(y) \in\left(J^{k} A^{m}\right)_{y}$ for each $i=1, \ldots, k$, then on the fiber $\widetilde{\mathbb{L}}_{k, y}:=\left(p \circ \pi_{0, k} \circ \nu_{k}\right)^{-1}(y)$ of $p \circ \pi_{0, k} \circ \nu_{k}: \widetilde{\mathbb{L}}_{k} \rightarrow Y$, one has

$$
\widetilde{\omega}_{\log }\left(s_{1}, \ldots, s_{k}\right)_{\widetilde{\mathbb{L}}_{k, y}}=\widetilde{\omega}_{\log }\left(s_{1}^{\prime}, \ldots, s_{k}^{\prime}\right)_{\widetilde{\mathbb{R}}_{k, y}} .
$$


Proof. Define $\mathbb{L}_{k, x}$ to be the fiber $\left(\pi_{0, k}\right)^{-1}(x)$ of $\pi_{0, k}: \mathbb{L}_{k} \rightarrow \mathbb{L}$. Note that the natural morphism

$$
E_{k, k^{\prime}} \Omega_{\mathbb{L}}(\log Y) \otimes k(x) \stackrel{\simeq}{\rightarrow}\left(\pi_{0, k}\right)_{*} \mathscr{O}_{\mathbb{L}_{k}}\left(k^{\prime}\right) \otimes k(x) \rightarrow H^{0}\left(\mathbb{L}_{k, x}, \mathscr{O}_{\mathbb{L}_{k}}\left(k^{\prime}\right)_{\mid \mathbb{L}_{k, x}}\right)
$$

is an isomorphism, where $k(x)$ is the residue field of $\mathbb{L}$ at $x$. By the assumption that $j^{k} s_{i}(y)=$ $j^{k} s_{i}^{\prime}(y)$ for each $i=1, \ldots, k$, one has

$$
j^{k} s_{1} \wedge \cdots \wedge j^{k} s_{k}(y)=j^{k} s_{1}^{\prime} \wedge \cdots \wedge j^{k} s_{k}^{\prime}(y) \in \bigwedge^{k} J^{k} A^{m} \otimes k(y) .
$$

Hence for any $x \in p^{-1}(y)$, one has

$j^{k} W_{k, \mathbb{L}, Y}\left(p^{*} j^{k} s_{1} \wedge \cdots \wedge p^{*} j^{k} s_{k}\right)(x)=j^{k} W_{k, \mathbb{L}, Y}\left(p^{*} j^{k} s_{1}^{\prime} \wedge \cdots \wedge p^{*} j^{k} s_{k}^{\prime}\right)(x) \in E_{k, k^{\prime}} \Omega_{\mathbb{L}}(\log Y) \otimes L^{k} \otimes k(x)$, and we conclude that $\omega_{\log }\left(s_{1}, \ldots, s_{k}\right)_{\uparrow \mathbb{L}_{k, y}}=\omega_{\log }\left(s_{1}^{\prime}, \ldots, s_{k}^{\prime}\right)_{\uparrow \mathbb{L}_{k, y}}$.

It now suffices to observe that the co-support of the ideal sheaf $\mathfrak{w}_{k, \mathbb{L}, Y}$ does not contain the fiber $\mathbb{L}_{k, x}$. Indeed, the announced statement will follow at once by continuity. To see this, it suffices to take coordinates $\left(z_{1}, \ldots, z_{n}\right)$ centered at $y=p(x)$ and consider the functions $z_{1}, \ldots, z_{1}^{k}$ in a neighborhood of $y$. A direct computation then shows that $\omega_{\log }\left(z_{1}, \ldots, z_{1}^{k}\right)_{\mid \mathbb{L}_{k, x}}$ is not identically zero, which implies the announced result.

\section{Main COnstructions}

3.1. Fermat type hypersurfaces and associated pairs. To begin with, we construct a family of hypersurfaces in $Y$ parametrized by certain Fermat type as in [Bro17]. Let $A$ be a very ample line bundle on $Y$. For an integer $N \geqslant n=\operatorname{dim}(Y)$, we fix $N+1$ sections in general position $\tau_{0}, \ldots, \tau_{N} \in H^{0}(Y, A)$. By "general position" we mean that the divisors defined by $\left(\tau_{j}=0\right)_{j=0, \ldots, N}$ are all smooth and meet transversally. For any two positive integers $\varepsilon, \delta$, set

$$
\mathbb{I}:=\left\{I=\left(i_{0}, \ldots, i_{N}\right)|| I \mid=\delta\right\}
$$

and

$$
\mathbf{a}:=\left(a_{I} \in H^{0}\left(Y, A^{\varepsilon}\right)\right)_{|I|=\delta} \in \mathbb{A}:=\bigoplus_{I \in \mathbb{I}} H^{0}\left(Y, A^{\varepsilon}\right) .
$$

For two positive integers $r$ and $k$ fixed later according to our needs, consider the family $\mathscr{D} \rightarrow \mathbb{A}$ of hypersurfaces in $|(\varepsilon+(r+k) \delta) A|$ defined by the zero locus of the bihomogenous sections

$$
\sigma(\mathbf{a})(y): y \mapsto \sum_{|I|=\delta} a_{I}(y) \tau(y)^{(r+k) I},
$$

where $\left(a_{I}\right)_{|I|=\delta}$ varies in the parameter space $\mathbb{A}$, and $\tau:=\left(\tau_{0}, \ldots, \tau_{N}\right)$. For any $\mathbf{a} \in \mathbb{A}$, let us write $D_{\mathbf{a}}$ for the fiber of the family $\mathscr{D} \rightarrow \mathbb{A}$.

Write $m:=\varepsilon+(r+k) \delta$. Consider the total space $p: \mathbb{L} \rightarrow Y$ of $A^{m}$ defined in $\S 2.6$, and write $L:=p^{*} A^{m}$. With the same notation in loc. cit., consider the family of hypersurfaces $\mathscr{H} \rightarrow \mathbb{A}$ in $\mathbb{L}$ defined by the vanishing of the section

$$
T-p^{*} \sigma(\mathbf{a}) \in H^{0}(\mathbb{L}, L) .
$$

For any $\mathbf{a} \in \mathbb{A}$, write $H_{\mathbf{a}}:=\left(T-p^{*} \sigma(\mathbf{a})=0\right) \subset \mathbb{L}$. By [BD18] there exists a non-empty Zariski open subset $\mathbb{A}_{\text {sm }} \subset \mathbb{A}$ such that $D_{\mathbf{a}}$ is a smooth hypersurface for any a $\in \mathbb{A}_{\text {sm }}$, and so is $H_{\mathbf{a}}$. Let us now shrink the family $\mathscr{H}$ (resp. $\mathscr{D}$ ) to $\mathbb{A}_{\mathrm{sm}}$, and let us denote abusively $\mathscr{H} \rightarrow \mathbb{A}_{\mathrm{sm}}$ (resp. $\mathscr{D} \rightarrow \mathbb{A}_{\mathrm{sm}}$ ) this restricted family. Since we can see $\mathscr{D}$ as a hypersurface in $\mathscr{H}$ defined by the equation $(T=0)$. Then, by the choice of $\mathbb{A}_{\mathrm{sm}}, \mathscr{D}$ is a smooth hypersurface of $\mathscr{H}$ and moreover, $(\mathscr{H}, \mathscr{D}) \rightarrow \mathbb{A}_{\mathrm{sm}}$ is a smooth family of log pairs.

Let us define $\mathscr{H}_{k}$ to be the log Demailly $k$-jet tower of $\left(\mathscr{H}, \mathscr{D}, T_{\mathscr{H} / \mathbb{A}_{\text {sm }}}(-\log \mathscr{D})\right)$. Under the natural inclusive morphism

$$
\begin{aligned}
\mathbb{A}_{\mathrm{sm}} & \mapsto \mathbb{A}^{\mathrm{s}} \\
\mathbf{a} & \mapsto \sigma(\mathbf{a})
\end{aligned}
$$


to the universal family defined in $\S 2.7$, and by the flat base change theorem, one simply obtains $\mathscr{H}_{k}:=\mathscr{H}_{k}^{\mathrm{s}} \times_{\mathbb{A}^{\mathrm{s}}} \mathbb{A}_{\mathrm{sm}}$. Let us define

$$
\widetilde{\mathscr{H}_{k}}:=\widetilde{\mathscr{H}}_{k}^{\mathrm{s}} \times_{\mathbb{A}^{\mathrm{s}}} \mathbb{A}_{\mathrm{sm}}
$$

For any a $\in \mathbb{A}_{\mathrm{sm}}$, the fibers of $\mathscr{H}_{k} \rightarrow \mathbb{A}_{\mathrm{sm}}$ and $\widetilde{\mathscr{H}}_{k} \rightarrow \mathbb{A}_{\mathrm{sm}}$ are denoted by $H_{\mathbf{a}, k}$ and $\widetilde{H}_{\mathbf{a}, k}$ respectively. Observe that in view of Lemma 2.8, this notation is consistent in the sense that $\widetilde{H}_{\mathbf{a}, k}$ is indeed the blow-up of $H_{\mathbf{a}, k}$ along the logarithmic Wronskian ideal sheaf $\mathfrak{w}_{H_{\mathbf{a}, k}}$.

3.2. Mapping to the Grassmannians. Consider the log pair $(\mathbb{L}, Y)$ defined in $\S 3.1$ equipped with the line bundle $L:=p^{*} A^{m}$. By (2.2.3), one can define the higher order logarithmic connection $\nabla^{j}: L \rightarrow E_{j, j}^{\mathrm{GG}} \Omega_{\mathbb{L}}(\log Y) \otimes L$. As in $\S 2.4$, let us take a trivialization tower $\mathfrak{U}=$ $\left(\left(U, T_{U}\right),\left(U_{j}, \xi_{j}\right)_{1 \leqslant j \leqslant k}\right)$ of order $k$. A straightforward induction implies the following

Lemma 3.1. For any $I \in \mathbb{I}$ and for any $1 \leqslant j \leqslant k$, there exist $\mathbb{C}$-linear maps

$$
\begin{aligned}
\nabla_{I}^{j} & : H^{0}\left(Y, A^{\varepsilon}\right) \rightarrow H^{0}\left(\mathbb{L}, E_{j, j}^{\mathrm{GG}} \Omega_{\mathbb{L}}(\log Y) \otimes p^{*} A^{\varepsilon+k \delta}\right), \\
\nabla_{\mathfrak{U}, I}^{j}: H^{0}\left(Y, A^{\varepsilon}\right) & \rightarrow \mathscr{O}\left(U_{k}\right),
\end{aligned}
$$

such that for any $a_{I} \in H^{0}\left(Y, A^{\varepsilon}\right)$, one has

$$
\begin{aligned}
& \nabla^{j}\left(p^{*} a_{I} \cdot\left(p^{*} \tau\right)^{(r+k) I}\right)=\left(p^{*} \tau\right)^{r I} \cdot \nabla_{I}^{j}\left(a_{I}\right), \\
& \nabla_{\mathfrak{U}}^{j}\left(p^{*} a_{I} \cdot\left(p^{*} \tau\right)^{(r+k) I}\right)=\left(\tau_{U_{k}}\right)^{r I} \cdot \nabla_{\mathfrak{U}, I}^{j}\left(a_{I}\right) .
\end{aligned}
$$

Here we denote by $\tau_{U_{k}}$ the pull-back of trivialization of $p^{*} \tau$ under $L_{\uparrow U}$ to $U_{k}$.

Therefore, for any $I_{1}, \ldots, I_{k} \in \mathbb{I}$ and any $a_{I_{1}}, \ldots, a_{I_{k}} \in H^{0}\left(Y, L^{\varepsilon}\right)$ one can define

$$
W_{\log , I_{1}, \ldots, I_{k}}\left(a_{I_{1}}, \ldots, a_{I_{k}}\right):=\left|\begin{array}{ccc}
\nabla_{I_{1}}^{1}\left(a_{I_{1}}\right) & \cdots & \nabla_{I_{k}}^{1}\left(a_{I_{k}}\right) \\
\vdots & \ddots & \vdots \\
\nabla_{I_{1}}^{k}\left(a_{I_{1}}\right) & \cdots & \nabla_{I_{k}}^{k}\left(a_{I_{k}}\right)
\end{array}\right| \in H^{0}\left(\mathbb{L}, E_{k, k^{\prime}} \Omega_{\mathbb{L}}(\log Y) \otimes p^{*} A^{k(\varepsilon+k \delta)}\right) .
$$

It then follows from Lemma 3.1 that

$$
W_{\mathbb{L}, Y}\left(p^{*} a_{I_{1}} \cdot\left(p^{*} \tau\right)^{(r+k) I_{1}}, \ldots, p^{*} a_{I_{k}} \cdot\left(p^{*} \tau\right)^{(r+k) I_{k}}\right)=\left(p^{*} \tau\right)^{r\left(I_{1}+\cdots+I_{k}\right)} \cdot W_{\log , I_{1}, \ldots, I_{k}}\left(a_{I_{1}}, \ldots, a_{I_{k}}\right) .
$$

Set

$$
\omega_{\log , I_{1}, \ldots, I_{k}}\left(a_{I_{1}}, \ldots, a_{I_{k}}\right) \in H^{0}\left(\mathbb{L}_{k}, \mathscr{O}_{\mathbb{L}_{k}}\left(k^{\prime}\right) \otimes\left(p \circ \pi_{0, k}\right)^{*} A^{k(\varepsilon+k \delta)}\right),
$$

to be the inverse image of $\left(\pi_{0, k}\right)_{*}$ under the isomorphism (1.4.13), then

$$
\omega_{\log }\left(a_{I_{1}} \tau^{(r+k) I_{1}}, \ldots, a_{I_{k}} \tau^{(r+k) I_{k}}\right)=\omega_{\log , I_{1}, \ldots, I_{k}}\left(a_{I_{1}}, \ldots, a_{I_{k}}\right) \cdot\left(p \circ \pi_{0, k}\right)^{*} \tau^{r\left(I_{1}+\cdots+I_{k}\right)} .
$$

Moreover from Proposition 2.6 one can deduce at once

Lemma 3.2. The section $\omega_{\log , I_{1}, \ldots, I_{k}}\left(a_{I_{1}}, \ldots, a_{I_{k}}\right)$ vanishes along $\mathfrak{w}_{k, \mathbb{L}, Y}$. In other words,

$$
\omega_{\log , I_{1}, \ldots, I_{k}}\left(a_{I_{1}}, \ldots, a_{I_{k}}\right) \in H^{0}\left(\mathbb{L}_{k}, \mathscr{O}_{\mathbb{L}_{k}}\left(k^{\prime}\right) \otimes\left(p \circ \pi_{0, k}\right)^{*} A^{k(\varepsilon+k \delta)} \otimes \mathfrak{w}_{k, \mathbb{L}, Y}\right),
$$

where $\mathfrak{w}_{k, \mathbb{L}, Y}$ is the ideal sheaf defined in $\S 2.6$.

Proof. By (3.2.1), one has

$$
W_{\log , I_{1}, \ldots, I_{k}}\left(a_{I_{1}}, \ldots, a_{I_{k}}\right) \in H^{0}\left(\mathbb{L}, \mathscr{W}_{k, \mathbb{L}, Y} \otimes p^{*} A^{-r k \delta}\right),
$$

where we recall that $\mathscr{W}_{k, \mathbb{L}, Y}$ is the image of the morphism $j^{k} W_{\mathbb{L}, Y}$ defined in (2.6.21). Then the base ideal of $\omega_{\log , I_{1}, \ldots, I_{k}}\left(a_{I_{1}}, \ldots, a_{I_{k}}\right)$ belongs to the ideal sheaf of $\mathscr{O}_{\mathbb{L}_{k}}$ defined by the image of the morphism

$\pi_{0, k}^{-1}\left(\mathscr{W}_{k, \mathbb{L}, Y} \otimes p^{*} A^{-r k \delta}\right) \otimes\left(p \circ \pi_{0, k}\right)^{*} A^{-k(\varepsilon+k \delta)} \otimes \mathscr{O}_{\mathbb{L}_{k}}\left(-k^{\prime}\right) \otimes \hookrightarrow \pi_{0, k}^{*} E_{k, k^{\prime}} \Omega_{\mathbb{L}}(\log Y) \otimes \mathscr{O}_{\mathbb{L}_{k}}\left(-k^{\prime}\right) \rightarrow \mathscr{O}_{\mathbb{L}_{k}}$.

Note that the image of the above morphism coincides with that of the following one

$$
\pi_{0, k}^{*} \mathscr{W}_{k, \mathbb{L}, Y} \otimes \pi_{0, k}^{*} L^{-k} \otimes \mathscr{O}_{\mathbb{L}_{k}}\left(-k^{\prime}\right) \otimes \hookrightarrow \pi_{0, k}^{*} E_{k, k^{\prime}} \Omega_{\mathbb{L}}(\log Y) \otimes \mathscr{O}_{\mathbb{L}_{k}}\left(-k^{\prime}\right) \rightarrow \mathscr{O}_{\mathbb{L}_{k}}
$$


The lemma follows immediately from Proposition 2.6.

Recall that we define $\nu_{k}: \widetilde{\mathbb{L}}_{k} \rightarrow \mathbb{L}_{k}$ to be the blow-up of the ideal sheaf $\mathfrak{w}_{k, \mathbb{L}, Y}$. By Lemma 3.2 and (2.7.25) there exists a unique

$$
\widetilde{\omega}_{\log , I_{1}, \ldots, I_{k}}\left(a_{I_{1}}, \ldots, a_{I_{k}}\right) \in H^{0}\left(\widetilde{\mathbb{L}}_{k}, \nu_{k}^{*}\left(\mathscr{O}_{\mathbb{L}_{k}}\left(k^{\prime}\right) \otimes\left(p \circ \pi_{0, k}\right)^{*} A^{k(\varepsilon+k \delta)}\right) \otimes \mathscr{O}_{\widetilde{\mathbb{L}}_{k}}(-F)\right)
$$

such that

$$
\nu_{k}^{*} \omega_{\log , I_{1}, \ldots, I_{k}}\left(a_{I_{1}}, \ldots, a_{I_{k}}\right)=F \cdot \widetilde{\omega}_{\log , I_{1}, \ldots, I_{k}}\left(a_{I_{1}}, \ldots, a_{I_{k}}\right) .
$$

By definition $\omega_{\log , I_{1}, \ldots, I_{k}}\left(a_{I_{1}}, \ldots, a_{I_{k}}\right)$ is alternating with respect to $\left(I_{1}, \ldots, I_{k}\right)$. We then can define a rational map

$$
\begin{aligned}
\Phi: \mathbb{A} \times \mathbb{L}_{k} & \rightarrow \mathrm{P}\left(\Lambda^{k}\left(\mathbb{C}^{\mathbb{I}}\right)\right) \\
(\mathbf{a}, w) & \mapsto\left[\left(\omega_{\log , I_{1}, \ldots, I_{k}}\left(a_{I_{1}}, \ldots, a_{I_{k}}\right)(w)\right)_{I_{1}, \ldots, I_{k} \in \mathbb{I}}\right] .
\end{aligned}
$$

The map $\Phi$ can also be interpreted explicitly using our intrinsic construction in $\S 2.4$. Let us fix a tower trivialization $\mathfrak{U}$ of order $k$. If we denote by

$$
\nabla_{\mathfrak{U}, \bullet}^{i}(\mathbf{a}, w):=\left(\nabla_{\mathfrak{U}, I}^{j}\left(a_{I}\right)(w)\right)_{I \in \mathbb{I}} \in \bigoplus_{I \in \mathbb{I}} \mathscr{O}\left(\mathbb{A} \times U_{k}\right)
$$

for any $1 \leqslant i \leqslant k$, then we can define another rational map locally by

$$
\begin{aligned}
\Phi_{\mathfrak{U}}: \mathbb{A} \times U_{k} & \rightarrow \operatorname{Gr}_{k}\left(\mathbb{C}^{\mathbb{I}}\right) \\
(\mathbf{a}, w) & \mapsto \operatorname{Span}\left(\nabla_{\mathfrak{U}, \bullet}^{1}(\mathbf{a}, w), \cdots, \nabla_{\mathfrak{U}, \bullet}^{k}(\mathbf{a}, w)\right)_{I_{1}, \ldots, I_{k} \in \mathbb{I}}
\end{aligned}
$$

and this is indeed the localization of $\Phi$.

Lemma 3.3. One has $\Phi_{\mid \mathbb{A} \times U_{k}}=$ Pluc $\circ \Phi_{\mathfrak{U}}$, where Pluc : $\operatorname{Gr}_{k}\left(\mathbb{C}^{\mathbb{I}}\right) \hookrightarrow \mathrm{P}\left(\Lambda^{k}\left(\mathbb{C}^{\mathbb{I}}\right)\right)$ denotes the Plücker embedding.

Proof. Let us define

$$
\omega_{\mathfrak{U}, I_{1}, \ldots, I_{k}}\left(a_{I_{1}}, \ldots, a_{I_{k}}\right):=\left|\begin{array}{ccc}
\nabla_{\mathfrak{U}, I_{1}}^{1}\left(a_{I_{1}}\right) & \cdots & \nabla_{\mathfrak{U}, I_{1}}^{1}\left(a_{I_{k}}\right) \\
\vdots & \ddots & \vdots \\
\nabla_{\mathfrak{U}, I_{1}}^{k}\left(a_{I_{1}}\right) & \cdots & \nabla_{\mathfrak{U}, I_{k}}^{k}\left(a_{I_{k}}\right)
\end{array}\right| \in \mathscr{O}\left(\mathbb{A} \times U_{k}\right),
$$

which corresponds to the Plücker coordinate of Pluc $\circ \Phi_{\mathfrak{U}}$. By Lemma 3.1, one has

$$
\omega_{\mathfrak{U}, I_{1}, \ldots, I_{k}}\left(a_{I_{1}}, \ldots, a_{I_{k}}\right) \cdot \tau_{U_{k}}^{r\left(I_{1}+\cdots+I_{k}\right)}=\left|\begin{array}{ccc}
\nabla_{\mathfrak{U}}^{1}\left(p^{*} a_{I_{1}} \cdot\left(p^{*} \tau\right)^{(r+k) I_{1}}\right) & \cdots & \nabla_{\mathfrak{U}}^{1}\left(p^{*} a_{I_{k}} \cdot\left(p^{*} \tau\right)^{(r+k) I_{k}}\right) \\
\vdots & \ddots & \vdots \\
\nabla_{\mathfrak{U}}^{k}\left(p^{*} a_{I_{1}} \cdot\left(p^{*} \tau\right)^{(r+k) I_{1}}\right) & \cdots & \nabla_{\mathfrak{U}}^{k}\left(p^{*} a_{I_{k}} \cdot\left(p^{*} \tau\right)^{\left.(r+k) I_{k}\right)}\right.
\end{array}\right| .
$$

It follows from Proposition 2.3 that under the trivialization of $\mathfrak{U}$, one has

$$
\omega_{\log }\left(a_{I_{1}} \tau^{(r+k) I_{1}}, \ldots, a_{I_{k}} \tau^{(r+k) I_{k}}\right)_{\mid U_{k}}=\omega_{\mathfrak{U}, I_{1}, \ldots, I_{k}}\left(a_{I_{1}}, \ldots, a_{I_{k}}\right) \cdot \tau_{U_{k}}^{r\left(I_{1}+\cdots+I_{k}\right)} \cdot \boldsymbol{\Gamma}_{k, \mathfrak{U}},
$$

here $\boldsymbol{\Gamma}_{k, \mathfrak{U}} \in \mathscr{O}\left(U_{k}\right)$ is the holomorphic function defining $\boldsymbol{\Gamma}_{k}$ via the trivialization of $\mathfrak{U}$. By (3.2.2) we conclude the proof of the lemma.

Remark 3.4. By the proof of Lemma 3.3, one can glue $\omega_{\mathfrak{U}, I_{1}, \ldots, I_{k}}\left(a_{I_{1}}, \ldots, a_{I_{k}}\right)$ together to obtain a global section

$$
\omega_{\log , I_{1}, \ldots, I_{k}}^{\prime}\left(a_{I_{1}}, \ldots, a_{I_{k}}\right) \in H^{0}\left(\mathbb{L}_{k}, \mathscr{O}_{\mathbb{L}_{k}}(k, k-1, \ldots, 1) \otimes\left(p \circ \pi_{0, k}\right)^{*} A^{k(\varepsilon+k \delta)}\right)
$$

such that

$$
\omega_{\log , I_{1}, \ldots, I_{k}}\left(a_{I_{1}}, \ldots, a_{I_{k}}\right)=\omega_{\log , I_{1}, \ldots, I_{k}}^{\prime}\left(a_{I_{1}}, \ldots, a_{I_{k}}\right) \cdot \Gamma_{k}
$$

It follows from (2.7.24) that

$$
\nu_{k}^{*} \omega_{\log , I_{1}, \ldots, I_{k}}^{\prime}\left(a_{I_{1}}, \ldots, a_{I_{k}}\right)=F^{\prime} \cdot \widetilde{\omega}_{\log , I_{1}, \ldots, I_{k}}\left(a_{I_{1}}, \ldots, a_{I_{k}}\right) .
$$


Consider the following rational map

$$
\begin{aligned}
\widetilde{\Phi}: \mathbb{A} \times \widetilde{\mathbb{L}}_{k} & \rightarrow \mathrm{P}\left(\Lambda^{k}\left(\mathbb{C}^{\mathbb{I}}\right)\right) \\
(\mathbf{a}, \tilde{w}) & \mapsto\left[\left(\widetilde{\omega}_{\log , I_{1}, \ldots, I_{k}}\left(a_{I_{1}}, \ldots, a_{I_{k}}\right)(\tilde{w})\right)_{I_{1}, \ldots, I_{k} \in \mathbb{I}}\right] .
\end{aligned}
$$

Since $\widetilde{\mathbb{L}}_{k} \backslash \operatorname{Supp}(F) \stackrel{\nu_{k}}{\rightarrow} \mathbb{L}_{k} \backslash \operatorname{Supp}\left(\mathscr{O}_{\mathbb{L}_{k}} / \mathfrak{w}_{k, \mathbb{L}, Y}\right)$ is a isomorphism, one has $\widetilde{\Phi}=\Phi \circ \nu_{k}$ outside $\operatorname{Supp}(F)$, and by the fact that $\widetilde{\mathbb{L}}_{k}$ is irreducible, this implies that $\widetilde{\Phi}$ also factors through the Plücker embedding, which is also denoted by $\widetilde{\Phi}$. One thus has the following commutative diagram

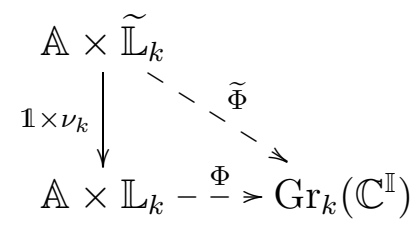

3.3. Partially resolving the indeterminacy. In this subsection, we will find a local and linear description for $\widetilde{\Phi}$, and use this to prove that $\nu_{k}$ partially resolves the indeterminacies of rational map $\widetilde{\Phi}$ in the same spirit as [Bro17, Lemmata $3.6 \& 3.7$ ].

Lemma 3.5. Fix any $\varepsilon \geqslant k$ and any $N>n$. For any $\tilde{w}_{0} \in \widetilde{\mathbb{L}}_{k}$, there exists an open neighborhood $\widetilde{U}_{\tilde{w}_{0}}$ of $\tilde{w}_{0}$ such that we can define $\mathbb{C}$-linear maps

$$
\ell_{I}^{p}: H^{0}\left(Y, A^{\varepsilon}\right) \rightarrow \mathscr{O}\left(\widetilde{U}_{\tilde{w}_{0}}\right)
$$

for any $I \in \mathbb{I}$ and $p=1, \ldots, k$ satisfying the following conditions.

(i) Write $\ell_{\bullet}^{p}(\mathbf{a}, \tilde{w}):=\left(\ell_{I}^{p}\left(a_{I}\right)(\tilde{w})\right)_{I \in \mathbb{I}} \in \mathbb{C}^{\mathbb{I}}$. The Plücker coordinates of $\widetilde{\Phi}(\mathbf{a}, \tilde{w})$ in $\operatorname{Gr}_{k}\left(\mathbb{C}^{\mathbb{I}}\right)$ all vanishes if and only

$$
\operatorname{dim} \operatorname{Span}\left(\ell_{\bullet}^{1}(\mathbf{a}, \tilde{w}), \ldots, \ell_{\bullet}^{k}(\mathbf{a}, \tilde{w})\right)<k .
$$

(ii) When $\operatorname{dim} \operatorname{Span}\left(\ell_{\bullet}^{1}(\mathbf{a}, \tilde{w}), \ldots, \ell_{\bullet}^{k}(\mathbf{a}, \tilde{w})\right)=k$, one has

$$
\widetilde{\Phi}(\mathbf{a}, \tilde{w})=\left(\ell_{\bullet}^{1}(\mathbf{a}, \tilde{w}), \ldots, \ell_{\bullet}^{k}(\mathbf{a}, \tilde{w})\right) .
$$

(iii) Set $y:=p \circ \pi_{0, k} \circ \nu_{k}\left(\tilde{w}_{0}\right) \in Y$ and define $\rho_{y}:\left(\mathbb{C}^{\mathbb{I}}\right)^{k} \rightarrow\left(\mathbb{C}^{\mathbb{I}_{y}}\right)^{k}$ to be the natural projection map, where

$$
\mathbb{I}_{y}:=\left\{I \in \mathbb{I} \mid \tau^{I}(y) \neq 0\right\}
$$

Define a linear map

$$
\begin{aligned}
\widetilde{\varphi}_{\tilde{w}_{0}}: \mathbb{A} & \rightarrow\left(\mathbb{C}^{\mathbb{I}}\right)^{k} \\
\mathbf{a} & \mapsto\left(\ell_{\bullet}^{1}\left(\mathbf{a}, \tilde{w}_{0}\right), \ldots, \ell_{\bullet}^{k}\left(\mathbf{a}, \tilde{w}_{0}\right)\right) .
\end{aligned}
$$

Then one has

$$
\operatorname{rank} \rho_{y} \circ \widetilde{\varphi}_{\tilde{w}_{0}}=k \cdot \# \mathbb{I}_{y},
$$

where $\# \mathbb{I}_{y}$ denotes to be the cardinality of $\mathbb{I}_{y}$.

Proof. Set $w_{0}:=\nu_{k}\left(\tilde{w}_{0}\right), x_{0}=\pi_{0, k}\left(w_{0}\right)$ and thus $y=p\left(x_{0}\right)$. Since $m:=\varepsilon+(r+k) \delta \geqslant k$, by Proposition 2.6 there exist $b_{1}, \ldots, b_{k} \in H^{0}\left(Y, A^{m}\right)$ such that $\tilde{\omega}_{\log }\left(b_{1}, \ldots, b_{k}\right)(\tilde{w}) \neq 0$ on some neighborhood $\widetilde{U}_{w_{0}}$ of $\tilde{w}_{0}$ in $\widetilde{\mathbb{L}}_{k}$. Pick a trivialization tower $\mathfrak{U}$ of order $k$ such that $w_{0} \in U_{k}$. We shrink $\widetilde{U}_{w_{0}}$ such that $\widetilde{U}_{w_{0}} \subset \nu_{k}^{-1}\left(U_{k}\right)$. For any $\sigma \in H^{0}\left(Y, A^{m}\right)$, one has $\nabla_{\mathfrak{U}}^{i}(\sigma) \in \mathscr{O}\left(U_{k}\right)$, thus by abuse of notation, we also write $\nabla_{\mathfrak{U}}^{i}(\sigma)$ as a holomorphic function on $\widetilde{U}_{\tilde{w}_{0}}$ under the pull-back $\nu_{k}: \widetilde{U}_{\tilde{w}_{0}} \rightarrow U_{k}$

It follows from Lemma 3.1 that, for any $p=1, \ldots, k$, one can define

$$
\omega_{\log , p, I}^{\prime}\left(b_{1}, \ldots, b_{p-1}, a_{I}, b_{p+1}, \ldots, b_{k}\right) \in H^{0}\left(\mathbb{L}_{k}, \mathscr{O}_{\mathbb{L}_{k}}(k, k-1, \ldots, 1) \otimes\left(p \circ \pi_{0, k}\right)^{*} A^{k m-r \delta}\right)
$$


such that

$$
\omega_{\log }^{\prime}\left(b_{1}, \ldots, b_{p-1}, a_{I} \cdot \tau^{(r+k) I}, b_{p+1}, \ldots, b_{k}\right)=\left(p \circ \pi_{0, k}\right)^{*} \tau^{r I} \cdot \omega_{\log , p, I}^{\prime}\left(b_{1}, \ldots, b_{p-1}, a_{I}, b_{p+1}, \ldots, b_{k}\right) .
$$

Indeed, locally $\omega_{\log , p, I}^{\prime}\left(b_{1}, \ldots, b_{p-1}, a_{I}, b_{p+1}, \ldots, b_{k}\right)$ is defined by

$$
\left|\begin{array}{ccccccc}
\nabla_{\mathfrak{U}}^{1}\left(b_{1}\right) & \cdots & \nabla_{\mathfrak{U}}^{1}\left(b_{p-1}\right) & \nabla_{\mathfrak{U}, I}^{1}\left(a_{I}\right) & \nabla_{\mathfrak{U}}^{1}\left(b_{p+1}\right) & \cdots & \nabla_{\mathfrak{U}}^{1}\left(b_{k}\right) \\
\vdots & \ddots & \vdots & \vdots & \vdots & \ddots & \vdots \\
\nabla_{\mathfrak{U}}^{k}\left(b_{1}\right) & \cdots & \nabla_{\mathfrak{U}}^{k}\left(b_{p-1}\right) & \nabla_{\mathfrak{U}, I}^{k}\left(a_{I}\right) & \nabla_{\mathfrak{U}}^{k}\left(b_{p+1}\right) & \cdots & \nabla_{\mathfrak{U}}^{k}\left(b_{k}\right)
\end{array}\right|
$$

By the relation between $\omega_{\log }(\bullet)$ and $\omega_{\log }^{\prime}(\bullet)$ in $\S 2.4$ and similar arguments as Lemma 3.2, one deduces that

$\omega_{\log , p, I}^{\prime}\left(b_{1}, \ldots, b_{p-1}, a_{I}, b_{p+1}, \ldots, b_{k}\right) \in H^{0}\left(\mathbb{L}_{k}, \mathscr{O}_{\mathbb{L}_{k}}(k, k-1, \ldots, 1) \otimes\left(p \circ \pi_{0, k}\right)^{*} A^{k m-r \delta} \otimes \mathfrak{w}_{k, \mathbb{L}, Y}^{\prime}\right)$, where $\mathfrak{w}_{k, \mathbb{L}, Y}^{\prime}$ is the ideal sheaf of $\mathbb{L}_{k}$ defined in $\S$ 2.6. By (2.7.26), there exists a unique holomorphic section

$\tilde{\omega}_{\log , p, I}\left(b_{1}, \ldots, b_{p-1}, a_{I}, b_{p+1}, \ldots, b_{k}\right) \in H^{0}\left(\widetilde{\mathbb{L}}_{k}, \nu_{k}^{*}\left(\mathscr{O}_{\mathbb{L}_{k}}(k, k-1, \ldots, 1) \otimes\left(p \circ \pi_{0, k}\right)^{*} A^{k m-r \delta}\right) \otimes \mathscr{O}_{\widetilde{\mathbb{L}}_{k}}\left(-F^{\prime}\right)\right)$

such that

$$
\nu_{k}^{*} \omega_{\log , p, I}^{\prime}\left(b_{1}, \ldots, b_{p-1}, a_{I}, b_{p+1}, \ldots, b_{k}\right)=F^{\prime} \cdot \tilde{\omega}_{\log , p, I}\left(b_{1}, \ldots, b_{p-1}, a_{I}, b_{p+1}, \ldots, b_{k}\right) .
$$

On $\widetilde{U}_{\tilde{w}_{0}}$, within the trivialization of $\mathfrak{U}$, we now define

$$
\begin{aligned}
\ell_{I}^{p}\left(a_{I}\right): & =\frac{\nu_{k}^{*} \omega_{\log , p, I}^{\prime}\left(b_{1}, \ldots, b_{p-1}, a_{I}, b_{p+1}, \ldots, b_{k}\right)}{\nu_{k}^{*} \omega_{\log }^{\prime}\left(b_{1}, \ldots, b_{k}\right)} \\
& =\frac{F^{\prime} \cdot \tilde{\omega}_{\log , p, I}\left(b_{1}, \ldots, b_{p-1}, a_{I}, b_{p+1}, \ldots, b_{k}\right)}{F^{\prime} \cdot \widetilde{\omega}_{\log }\left(b_{1}, \ldots, b_{k}\right)} \\
& =\frac{\tilde{\omega}_{\log , p, I}\left(b_{1}, \ldots, b_{p-1}, a_{I}, b_{p+1}, \ldots, b_{k}\right)}{\widetilde{\omega}_{\log }\left(b_{1}, \ldots, b_{k}\right)}
\end{aligned}
$$

where the second equality is due to (2.7.26). Hence $\ell_{I}^{p}\left(a_{I}\right)$ are all holomorphic functions over $\widetilde{U}_{\tilde{w}_{0}}$. Consider the matrix of functions $G(\tilde{w})$ over $\widetilde{U}_{\tilde{w}_{0}}$ defined by

$$
G(\tilde{w}):=\left(\begin{array}{ccc}
\nabla_{\mathfrak{U}}^{1}\left(b_{1}\right) & \ldots, & \nabla_{\mathfrak{U}}^{1}\left(b_{k}\right) \\
\vdots & \ddots & \vdots \\
\nabla_{\mathfrak{U}}^{k}\left(b_{1}\right) & \ldots & \nabla_{\mathfrak{U}}^{k}\left(b_{k}\right)
\end{array}\right)
$$

then by definition, one has

$$
G \cdot\left(\begin{array}{c}
\ell_{I}^{1}\left(a_{I}\right) \\
\vdots \\
\ell_{I}^{k}\left(a_{I}\right)
\end{array}\right)=\frac{\operatorname{det} G}{\nu_{k}^{*} \omega_{\log }^{\prime}\left(b_{1}, \ldots, b_{k}\right)} \cdot\left(\begin{array}{c}
\nabla_{\mathfrak{U}, I}^{1}\left(a_{I}\right) \\
\vdots \\
\nabla_{\mathfrak{U}, I}^{k}\left(a_{I}\right)
\end{array}\right)=\left(\begin{array}{c}
\nabla_{\mathfrak{U}, I}^{1}\left(a_{I}\right) \\
\vdots \\
\nabla_{\mathfrak{U}, I}^{k}\left(a_{I}\right)
\end{array}\right) .
$$

For any $I_{1}, \ldots, I_{k} \in \mathbb{I}$, on $\widetilde{U}_{\tilde{w}_{0}}$ one has

$$
\left|\begin{array}{ccc}
\ell_{I_{1}}^{1}\left(a_{I_{1}}\right) & \ldots & \ell_{I_{k}}^{1}\left(a_{I_{k}}\right) \\
\vdots & \ddots & \vdots \\
\ell_{I_{1}}^{k}\left(a_{I_{1}}\right) & \ldots & \ell_{I_{k}}^{k}\left(a_{I_{k}}\right)
\end{array}\right|=\frac{\nu_{k}^{*} \omega_{\log , I_{1}, \ldots, I_{k}}^{\prime}\left(a_{I_{1}}, \ldots, a_{I_{k}}\right)}{\nu_{k}^{*} \omega_{\log }^{\prime}\left(b_{1}, \ldots, b_{k}\right)}=\frac{\widetilde{\omega}_{\log , I_{1}, \ldots, I_{k}}\left(a_{I_{1}}, \ldots, a_{I_{k}}\right)}{\tilde{\omega}_{\log }\left(b_{1}, \ldots, b_{k}\right)},
$$

where the last equality is due to (2.7.26) and (3.2.4). This implies Lemmas 3.5.(i) and 3.5.(ii).

In order to prove Lemma 3.5.(iii), we first observe that the linear map $\widetilde{\varphi}_{\tilde{w}_{0}}$ is block with respect to $I \in \mathbb{I}$. Thus set

$$
\begin{aligned}
\tilde{\varphi}_{I}: H^{0}\left(Y, A^{\varepsilon}\right) & \rightarrow \mathbb{C}^{k} \\
a_{I} & \mapsto\left(\ell_{I}^{1}\left(a_{I}\right)\left(\tilde{w}_{0}\right), \ldots, \ell_{I}^{k}\left(a_{I}\right)\left(\tilde{w}_{0}\right)\right) .
\end{aligned}
$$


Note that $A^{\varepsilon}$ generates $k$-jets everywhere on $Y$ by the assumption that $\varepsilon \geqslant k$. For any $I \in \mathbb{I}_{y}$, by the definition of $\mathbb{I}_{y}$ one has $\tau^{I}(y) \neq 0$, and one can therefore take $c_{1}, \ldots, c_{k} \in H^{0}\left(Y, A^{\varepsilon}\right)$ such that the $k$-jets

for any $i=1, \ldots, k$. Then

$$
j^{k} c_{i}(y)=j^{k}\left(\frac{b_{i}}{\tau^{(r+k) I}}\right)(y)
$$

$$
j^{k} b_{i}(y)=j^{k}\left(c_{i} \cdot \tau^{(r+k) I}\right)(y)
$$

for any $i=1, \ldots, k$. It follows from Lemma 2.9 that

$$
\tilde{\omega}_{\log }\left(c_{1} \cdot \tau^{(r+k) I}, \ldots, c_{k} \cdot \tau^{(r+k) I}\right)\left(\tilde{w}_{0}\right)=\tilde{\omega}_{\log }\left(b_{1}, \ldots, b_{k}\right)\left(\tilde{w}_{0}\right) .
$$

Hence

$$
\begin{gathered}
\left|\begin{array}{ccc}
\ell_{I}^{1}\left(c_{1}\right) & \ldots & \ell_{I}^{1}\left(c_{k}\right) \\
\vdots & \ddots & \vdots \\
\ell_{I}^{k}\left(c_{1}\right) & \ldots & \ell_{I}^{k}\left(c_{k}\right)
\end{array}\right|\left(\tilde{w}_{0}\right) \stackrel{(3.3 .8)}{=} \frac{\widetilde{\omega}_{\log , I, \ldots, I}\left(c_{1}, \ldots, c_{k}\right)\left(\tilde{w}_{0}\right)}{\widetilde{\omega}_{\log }\left(b_{1}, \ldots, b_{k}\right)\left(\tilde{w}_{0}\right)} \\
\stackrel{(3.2 .2)}{=} \frac{\widetilde{\omega}_{\log }\left(c_{1} \cdot \tau^{(r+k) I}, \ldots, c_{k} \cdot \tau^{(r+k) I}\right)\left(\tilde{w}_{0}\right)}{\tau(y)^{k r I} \cdot \widetilde{\omega}_{\log }\left(b_{1}, \ldots, b_{k}\right)\left(\tilde{w}_{0}\right)} \\
=\frac{1}{\tau(y)^{k r I}} \neq 0 .
\end{gathered}
$$

This implies that $\operatorname{rank} \widetilde{\varphi}_{I}=k$. Lemma 3.5.(iii) immediately follows from that $\widetilde{\varphi}_{\tilde{w}_{0}}:=\bigoplus_{I \in \mathbb{I}} \widetilde{\varphi}_{I}$. We finish the proof of the whole lemma.

Let us apply Lemma 3.5 to show that $\tilde{\Phi}(\mathbf{a}, \bullet): \widetilde{\mathbb{L}}_{k} \rightarrow \operatorname{Gr}_{k}\left(\mathbb{C}^{\mathbb{I}}\right)$ is a regular morphism for general $\mathbf{a} \in \mathbb{A}$ when we choose the parameters $N, \delta, k$ properly.

Lemma 3.6. Assume that $N>n, \delta \geqslant(k+1) n+k$. Then there exists a Zariski dense open set $\mathbb{A}_{\text {def }} \subset \mathbb{A}_{\text {sm }}$ such that $\widetilde{\Phi}: \mathbb{A}_{\text {def }} \times \widetilde{\mathbb{L}}_{k} \rightarrow \operatorname{Gr}_{k}\left(\mathbb{C}^{\mathbb{I}}\right)$ is a regular morphism.

Proof. By (3.2.5) the indeterminacy locus of $\widetilde{\Phi}$ is contained in the subvariety

$$
Z:=\left\{(\mathbf{a}, \tilde{w}) \in \mathbb{A} \times \widetilde{\mathbb{L}}_{k} \mid \tilde{\omega}_{\log , I_{1}, \ldots, I_{k}}\left(a_{I_{1}}, \ldots, a_{I_{k}}\right)(\tilde{w})=0 \forall I_{1}, \ldots, I_{k} \in \mathbb{I}\right\} .
$$

Denote $\operatorname{pr}_{1}: \mathbb{A} \times \widetilde{\mathbb{L}}_{k} \rightarrow \mathbb{A}$ and $\operatorname{pr}_{2}: \mathbb{A} \times \widetilde{\mathbb{L}}_{k} \rightarrow \widetilde{\mathbb{L}}_{k}$ to be the projection maps. It then suffices to show that $\operatorname{pr}_{1}(Z) \subsetneq \mathbb{A}$. Fix any $\tilde{w}_{0} \in \widetilde{\mathbb{L}}_{k}$. Set $w_{0}:=\nu_{k}\left(\tilde{w}_{0}\right), x=\pi_{0, k}\left(w_{0}\right)$ and $y=p\left(x_{0}\right)$. Define $Z_{\tilde{w}_{0}}:=Z \cap \operatorname{pr}_{2}^{-1}\left(\tilde{w}_{0}\right)$. For the linear map defined in Lemma 3.5.(iii), it follows from Lemma 3.5.(i) that

where

$$
\operatorname{pr}_{1}\left(Z_{\tilde{w}_{0}}\right)=\left(\tilde{\varphi}_{\tilde{w}_{0}}\right)^{-1}(\Delta)
$$

$$
\Delta:=\left\{\left(v_{\bullet}^{1}, \ldots, v_{\bullet}^{k}\right) \in\left(\mathbb{C}^{\mathbb{I}}\right)^{k} \mid \operatorname{dim} \operatorname{Span}\left(v_{\bullet}^{1}, \ldots, v_{\bullet}^{k}\right)<k\right\} .
$$

Define a linear subspace of $\left(\mathbb{C}^{\mathbb{I}_{y}}\right)^{k}$ by

$$
\Delta_{y}:=\left\{\left(v_{\bullet}^{1}, \ldots, v_{\bullet}^{k}\right) \in\left(\mathbb{C}^{\mathbb{I}_{y}}\right)^{k} \mid \operatorname{dim} \operatorname{Span}\left(v_{\bullet}^{1}, \ldots, v_{\bullet}^{k}\right)<k\right\},
$$

and one has $\Delta \subseteq \rho_{y}^{-1}\left(\Delta_{y}\right)$, where $\rho_{y}:\left(\mathbb{C}^{\mathbb{I}}\right)^{k} \rightarrow\left(\mathbb{C}^{\mathbb{I}_{y}}\right)^{k}$ is the natural projection map. Hence

$$
\operatorname{pr}_{1}\left(Z_{\tilde{w}_{0}}\right) \subset\left(\rho_{y} \circ \tilde{\varphi}_{\tilde{w}_{0}}\right)^{-1} \Delta_{y},
$$

and

$$
\begin{aligned}
\operatorname{dim} Z_{\tilde{w}_{0}} & \leqslant \operatorname{dim} \operatorname{ker}\left(\rho_{y} \circ \tilde{\varphi}_{\tilde{w}_{0}}\right)+\operatorname{dim} \Delta_{y} \\
& =\operatorname{dim} \mathbb{A}-\operatorname{rank}\left(\rho_{y} \circ \tilde{\varphi}_{\tilde{w}_{0}}\right)+\operatorname{dim} \Delta_{y} \\
& \stackrel{(3.3 .7)}{=} \operatorname{dim} \mathbb{A}-k \# \mathbb{I}_{y}+(k-1)\left(\# \mathbb{I}_{y}+1\right) \\
& =\operatorname{dim} \mathbb{A}+(k-1)-\# \mathbb{I}_{y} .
\end{aligned}
$$


Therefore,

$$
\begin{aligned}
\operatorname{dim} Z & \leqslant \operatorname{dim} \widetilde{\mathbb{L}}_{k}+\max _{\tilde{w}_{0} \in \tilde{\mathbb{L}}_{k}} \operatorname{dim} Z_{\tilde{w}_{0}} \\
& \leqslant \operatorname{dim} \widetilde{\mathbb{L}}_{k}+\operatorname{dim} \mathbb{A}+(k-1)-\min _{y \in Y} \# \mathbb{I}_{y} \\
& \leqslant(k+1) n+1+(k-1)+\operatorname{dim} \mathbb{A}-(\delta+1) \\
& <\operatorname{dim} \mathbb{A} .
\end{aligned}
$$

Here we observe that $\# \mathbb{I}_{y} \geqslant\left(\begin{array}{c}N-n+\delta \\ \delta\end{array}\right) \geqslant \delta+1$ for any $y \in Y$ when $N>n$. Let us define $\mathbb{A}_{\text {def }}:=\left(\mathbb{A} \backslash \overline{\operatorname{pr}_{1}(Z)}\right) \cap \mathbb{A}_{\mathrm{sm}}$, which is a Zariski dense open set of $\mathbb{A}$. By the definition of $Z$, we conclude that $\tilde{\Phi}(\mathbf{a}, \bullet): \widetilde{\mathbb{L}}_{k} \rightarrow \mathrm{Gr}_{k}\left(\mathbb{C}^{\mathbb{I}}\right)$ is a regular morphism for any $\mathbf{a} \in \mathbb{A}_{\text {def }}$.

\section{Proof of the main Results}

4.1. Associated universal complete intersection variety. We are now in position to introduce the main geometric framework used during the proof of our main result. As in [BD18, Bro17, Den17, BD17] we rely on the universal complete intersection variety associated to our problem defined by

$$
\mathscr{Y}:=\left\{(\Delta,[z]) \in \operatorname{Gr}_{k}\left(H^{0}\left(\mathbb{P}^{k}, \mathscr{O}_{\mathbb{P}^{k}}(\delta)\right)\right) \times \mathbb{P}^{k} \mid \forall P \in \Delta, P([z])=0\right\},
$$

where we fix the parameter $N=k=n+1$ now. Let us write $\operatorname{Gr}_{k}:=\operatorname{Gr}_{k}\left(H^{0}\left(\mathbb{P}^{k}, \mathscr{O}_{\mathbb{P}^{k}}(\delta)\right)\right)$ for simplicity. For technical reasons, we will also need to adapt this construction to the stratification on $Y$ induced by the vanishings of the $\tau_{j}$ 's. To do this, let us define for any $J \subset\{0, \ldots, k\}$,

$$
\begin{aligned}
\mathbb{P}_{J} & :=\left\{[z] \in \mathbb{P}^{k} \mid z_{j}=0 \text { if } j \in J\right\}, \\
Y_{J} & :=\left\{y \in Y \mid \tau_{j}(y)=0 \Leftrightarrow j \in J\right\}, \\
\mathbb{I}_{J} & :=\{I \in \mathbb{I} \mid \operatorname{Supp}(I) \subset\{0, \ldots, k\} \backslash J\},
\end{aligned}
$$

and let us also consider the restricted universal complete intersection varieties

$$
\begin{aligned}
\mathscr{Y}_{J}: & =\left\{(\Delta,[z]) \in \mathrm{Gr}_{k} \times \mathbb{P}_{J} \mid \forall P \in \Delta, P([z])=0\right\} \\
& =\mathscr{Y} \cap\left(\mathrm{Gr}_{k} \times \mathbb{P}_{J}\right) \subset \mathscr{Y} .
\end{aligned}
$$

Let us denote by pr: $\mathscr{Y} \rightarrow \mathrm{Gr}_{k}$ the canonical projection, and for any $J \subset\{0, \ldots, k\}$ we set $\operatorname{pr}_{J}:=\operatorname{pr}_{\mathscr{Y}_{J}}: \mathscr{Y}_{J} \rightarrow \mathrm{Gr}_{k}$. Observe that the $\operatorname{pr}_{J}$ is generically finite. This observation is crucial in the rest of the argument which highly rests on the understanding of the geometry of the non-finite locus of $\mathrm{pr}_{J}$ :

$$
E_{J}:=\left\{y \in \mathscr{Y}_{J} \mid \operatorname{dim}_{y} \operatorname{pr}_{J}^{-1}\left(\operatorname{pr}_{J}(y)\right)>0\right\}
$$

and its image in $\mathrm{Gr}_{k}$ :

$$
G_{J}^{\infty}:=\operatorname{pr}_{J}\left(E_{J}\right)=\left\{\Delta \in \operatorname{Gr}_{k} \mid \operatorname{dim}_{p_{J}}^{-1}(\Delta)>0\right\} .
$$

4.2. Factorization through the universal complete intersection variety. Let us now relate this universal complete intersection to our special families of Fermat type pairs constructed in the previous sections by considering the morphism

$$
\begin{aligned}
\tilde{\Psi}: \mathbb{A}_{\text {def }} \times \tilde{\mathbb{L}}_{k} & \rightarrow \operatorname{Gr}_{k} \times \mathbb{P}^{k} \\
(\mathbf{a}, \tilde{w}) & \mapsto\left(\tilde{\Phi}(\mathbf{a}, \tilde{w}),\left[\tau_{0}^{r}\left(p \circ \pi_{0, k} \circ \nu_{k}\right)(\tilde{w}), \ldots, \tau_{k}^{r}\left(p \circ \pi_{0, k} \circ \nu_{k}\right)(\tilde{w})\right]\right) .
\end{aligned}
$$

For any $J \subset\{0, \ldots, k\}$ let us write,

$$
\mathbb{L}_{k, J}:=\left(p \circ \pi_{0, k}\right)^{-1}\left(Y_{J}\right) \text { and } \widetilde{\mathbb{L}}_{k, J}:=\nu_{k}^{-1}\left(\mathbb{L}_{k, J}\right) .
$$


Recall the definition of the (restricted) families $\mathscr{H}_{k} \rightarrow \mathbb{A}_{\text {def }}$ and $\widetilde{\mathscr{H}}_{k} \rightarrow \mathbb{A}_{\text {def }}$ and denote, for any $J \subset\{0, \ldots, k\}$,

$$
\mathscr{H}_{k, J}=\mathscr{H}_{k} \cap\left(\mathbb{A}_{\text {def }} \times \widetilde{\mathbb{L}}_{k, J}\right) \text { and } \widetilde{\mathscr{H}}_{k, J}=\widetilde{\mathscr{H}}_{k} \cap\left(\mathbb{A}_{\text {def }} \times \widetilde{\mathbb{L}}_{k, J}\right) .
$$

For any $\mathbf{a} \in \mathbb{A}_{\text {def }}$, let us denote by $H_{\mathbf{a}, k, J}$ and $\widetilde{H}_{\mathbf{a}, k, J}$ the fiber above a of $\mathscr{H}_{k, J}$ and $\widetilde{\mathscr{H}}_{k, J}$ respectively. One then has the crucial factorizing property of $\widetilde{\Psi}$.

Lemma 4.1. For any $J \subset\{0, \ldots, k\}$, when restricted to $\widetilde{\mathscr{H}}_{k, J}$, the morphism $\widetilde{\Psi}$ factors through $\mathscr{Y}_{J} \subset \mathrm{Gr}_{k} \times \mathbb{P}^{k}$.

Proof. It suffices to prove that $\widetilde{\Psi}$ restricted to $\widetilde{\mathbb{L}}_{k, J} \times \mathbb{A}_{\text {def }}$ factors through $\mathrm{Gr}_{k} \times \mathbb{P}_{J}$ and that $\widetilde{\Psi}$ restricted to $\widetilde{\mathscr{H}}_{k}$ factors through $\mathscr{Y}$. The first claim is straightforward to prove. For the second one, since $\widetilde{\Phi}=\Phi \circ \nu_{k}$, it suffices to prove that the rational map

$$
\begin{aligned}
\Psi: \mathbb{A}_{\text {def }} \times \mathbb{L}_{k} & \rightarrow \operatorname{Gr}_{k} \times \mathbb{P}^{k} \\
(\mathbf{a}, w) & \mapsto\left(\Phi(\mathbf{a}, w),\left[\tau_{0}^{r}\left(\left(p \circ \pi_{0, k}\right)(w)\right), \ldots, \tau_{k}^{r}\left(\left(p \circ \pi_{0, k}\right)(w)\right)\right]\right)
\end{aligned}
$$

factors through $\mathscr{Y}$ when restricted to $\mathscr{H}_{k}$.

Let us take a trivialization tower $\mathfrak{U}$ of order $k$ as in $\S 2.4$. Pick any $\mathbf{a} \in \mathbb{A}_{\text {def }}$. Recall that $H_{\mathbf{a}}$ is defined by the vanishing of the section

$$
T-p^{*} \sigma(\mathbf{a})=T-p^{*}\left(\sum_{|I|=\delta} a_{I} \cdot \tau^{(r+k) I}\right) \in H^{0}\left(\mathbb{L}, p^{*} L\right) .
$$

Then over $H_{\mathbf{a}, k} \cap U_{k}$, for any $i=1, \ldots, k$ one has

$$
0=\nabla_{\mathfrak{U}}^{i}\left(T-p^{*} \sigma(\mathbf{a})\right) \stackrel{(2.2 .4)}{=}-\nabla_{\mathfrak{U}}^{i} p^{*} \sigma(\mathbf{a})=-\sum_{|I|=\delta}\left(\tau_{U_{k}}\right)^{r I} \cdot \nabla_{\mathfrak{U}, I}^{j}\left(a_{I}\right),
$$

where the last equality is due to Lemma 3.1, and we denote by $\tau_{U_{k}}$ the pull-back of trivialization of $\tau$ under $L_{\uparrow U}$ to $U_{k}$. By the alternative definition of $\Phi$ in Lemma 3.3, we conclude the first claim. The second claim of the lemma follows directly from Lemma 3.6.

4.3. An effective Nakamaye type result. Let us denote by $\mathrm{pr}_{1}, \mathrm{pr}_{2}$ the projection on the first and second factor of $\mathrm{Gr}_{k} \times \mathbb{P}^{k}$, and let us consider the Plücker line bundle $\mathscr{L}$ on $\mathrm{Gr}_{k}$. By definition, one has $\mathscr{L}:=\operatorname{Pluc}^{*} \mathscr{O}_{\mathrm{P}\left(\Lambda^{k}\left(\mathbb{C}^{\mathbb{I}}\right)\right)}(1)$ where Pluc $: \operatorname{Gr}_{k} \hookrightarrow \mathrm{P}\left(\Lambda^{k}\left(\mathbb{C}^{\mathbb{I}}\right)\right)$ denotes the Plücker embedding. The use of the universal complete intersection in our situation is justified by the following formula: for any $d \in \mathbb{N}$, one has

$$
\widetilde{\Psi}^{*}\left(\mathscr{L}^{d} \otimes \mathscr{O}_{\mathbb{P}^{k}}(-1)\right)=\nu_{k}^{*}\left(\mathscr{O}_{\mathbb{L}_{k}}\left(d k^{\prime}\right) \otimes\left(p \circ \pi_{0, k}\right)^{*} A^{d k(\varepsilon+k \delta)-r}\right) \otimes \mathscr{O}_{\widetilde{\mathbb{L}}_{k}}(-d F) .
$$

where we write $\mathscr{L}^{d} \otimes \mathscr{O}_{\mathbb{P}^{k}}(-1):=\operatorname{pr}_{1}^{*} \mathscr{L}^{d} \otimes \operatorname{pr}_{2}^{*} \mathscr{O}_{\mathbb{P}^{k}}(-1)$. In particular, if $r>d k(\varepsilon+k \delta)$, every global section of $\mathscr{L}^{d} \otimes \mathscr{O}_{\mathbb{P}^{k}}(-1)$ gives rise to a logarithmic jet differentials vanishing along an ample divisor of $Y$. Of course, there may not exist such global sections due to the presence of the negative twist $\operatorname{pr}_{2}^{*} \mathscr{O}_{\mathbb{P} k}(-1)$. However, observe that the line bundle $\mathscr{L}$ is ample on $\mathrm{Gr}_{k}$ and the projection $\operatorname{pr}_{J}: \mathscr{Y}_{J} \rightarrow \mathrm{Gr}_{k}$ is generically finite, therefore, $\operatorname{pr}_{J}^{*} \mathscr{L}=\operatorname{pr}^{*} \mathscr{L}_{\uparrow \mathscr{Y}_{J}}$ is big and nef for any $J \subset\{0, \ldots, k\}$ and therefore, for $d$ large enough, there are many global sections of the line bundle $\mathscr{L}^{d} \otimes \mathscr{O}_{\mathbb{P} k}(-1)_{\mid \mathscr{Y}_{J}}$. In view of the factorization property established in the previous section, we obtain that for any $J \subset\{0, \ldots, k\}$, any integer $m$ and any $\mathbf{a} \in \mathbb{A}_{\text {def }}$,

$\operatorname{Bs}\left(\nu_{k}^{*}\left(\mathscr{O}_{H_{\mathbf{a}, k, J}}\left(d k^{\prime}\right) \otimes\left(p \circ \pi_{0, k}\right)^{*} A^{d k(\varepsilon+k \delta)-r}\right) \otimes \mathscr{O}_{\widetilde{H}_{\mathbf{a}, k, J}}(-d F)\right) \subseteq \widetilde{\Psi}^{-1}\left(\operatorname{Bs}\left(\mathscr{L}^{d} \otimes \mathscr{O}_{\mathbb{P}^{k}}(-1)_{\vdash} \mathscr{Y} J_{J}\right)\right)$.

These considerations lead us to study the right hand side in this formula. Since $\operatorname{pr}_{J}^{*} \mathscr{L}$ is big and nef for any $J \subset\{0, \ldots, k\}$, Nakamaye's theorem [Nak00] on the augmented base locus guaranties that

$$
E_{J}=\mathbf{B}_{+}\left(\operatorname{pr}_{J}^{*} \mathscr{L}\right)=\operatorname{Bs}\left(\mathscr{L}^{d} \otimes \mathscr{O}_{\mathbb{P} k}(-1) \mathscr{Y}_{J}\right)
$$


for $d$ large enough. To determine an explicit bound for the values of $d$ satisfying this formula is critical in order to obtain an effective bound on the degree in our main theorem. While we don't know a bound for this exact problem, the second named author was able in [Den17] to obtain the following bound for a slightly weaker inclusion sufficient for our purposes.

Theorem 4.2 ([Den17]). For any $d \geqslant \delta^{k-1}$, and any $J \subset\{0, \ldots, k\}$, the base locus of the line bundle $\mathscr{L}^{d} \otimes \mathscr{O}_{\mathbb{P}^{k}}(-1) \mathscr{Y}_{J}$ satisfies

$$
\operatorname{Bs}\left(\mathscr{L}^{d} \otimes \mathscr{O}_{\mathbb{P}^{k}}(-1) \mathscr{Y}_{J}\right) \subset \operatorname{pr}_{J}^{-1}\left(G_{J}^{\infty}\right)
$$

Recall that $G_{J}^{\infty}$ is the set of points in $\mathrm{Gr}_{k}$ such that the fiber of $\mathrm{pr}_{J}: \mathscr{Y}_{J} \rightarrow \mathrm{Gr}_{k}$ is not a finite set.

4.4. Avoiding the exceptional locus. In this section we explain how one can control $\widetilde{\Psi}^{-1}\left(\mathrm{pr}_{J}^{-1}\left(G_{J}^{\infty}\right)\right)$.

Lemma 4.3. For any $J \subset\{0, \ldots, k\}$, when $\delta \geqslant n(k+1)+1$, there exists a non-empty Zariski open subset $\mathbb{A}_{J} \subset \mathbb{A}_{\text {def }}$ such that

$$
\widetilde{\Phi}^{-1}\left(G_{J}^{\infty}\right) \cap\left(\mathbb{A}_{J} \times \tilde{\mathbb{L}}_{k, J}\right)=\varnothing .
$$

Proof. Take any $\tilde{w}_{0} \in \widetilde{\mathbb{L}}_{k, J}$. Set $w_{0}:=\nu_{k}\left(\tilde{w}_{0}\right), x=\pi_{0, k}\left(w_{0}\right)$ and $y=p\left(x_{0}\right)$. Then we have $\mathbb{I}_{J}=\mathbb{I}_{y}$, and we define the following analogues of $\mathscr{Y}$ parametrized by affine spaces

$$
\begin{gathered}
\widetilde{\mathscr{Y}}_{J}:=\left\{\left(P_{1}, \ldots, P_{k},[z]\right) \in\left(\mathbb{C}^{\mathbb{I}}\right)^{k} \times \mathbb{P}_{J} \mid P_{1}([z])=\cdots=P_{k}([z])=0\right\}, \\
\widetilde{\mathscr{Y}_{y}}:=\left\{\left(P_{1}, \ldots, P_{k},[z]\right) \in\left(\mathbb{C}^{\mathbb{I}_{J}}\right)^{k} \times \mathbb{P}_{J} \mid P_{1}([z])=\cdots=P_{k}([z])=0\right\} .
\end{gathered}
$$

Here we use the identification $\mathbb{C}^{\mathbb{I}} \cong H^{0}\left(\mathbb{P}^{k}, \mathscr{O}_{\mathbb{P} k}(\delta)\right)$ and $\mathbb{C}^{\mathbb{I}} \cong H^{0}\left(\mathbb{P}_{J}, \mathscr{O}_{\mathbb{P}_{J}}(\delta)\right)$. By analogy with $G_{J}^{\infty}$, we denote by $V_{1, J}^{\infty}$ (resp. $\left.V_{2, J}^{\infty}\right)$ the set of points in $\left(\mathbb{C}^{\mathbb{I}}\right)^{k}$ (resp. $\left.\left(\mathbb{C}^{\mathbb{I}}\right)^{k}\right)$ at which the fiber in $\widetilde{\mathscr{Y}_{J}}$ (resp. $\widetilde{\mathscr{Y}_{y}}$ ) is positive dimensional.

We take the linear map $\tilde{\varphi}_{\tilde{w}_{0}}: \mathbb{A} \rightarrow\left(\mathbb{C}^{\mathbb{I}}\right)^{k}$

$$
\begin{aligned}
\tilde{\varphi}_{\tilde{w}_{0}}: \mathbb{A} & \rightarrow\left(\mathbb{C}^{\mathbb{I}}\right)^{k} \\
\mathbf{a} & \mapsto\left(\ell_{\bullet}^{1}\left(\mathbf{a}, \tilde{w}_{0}\right), \ldots, \ell_{\bullet}^{k}\left(\mathbf{a}, \tilde{w}_{0}\right)\right)
\end{aligned}
$$

defined in Lemma 3.5 so that, for any $\mathbf{a} \in \mathbb{A}_{\text {def }}$, we have

$$
\tilde{\Phi}\left(\mathbf{a}, \tilde{w}_{0}\right)=\operatorname{Span}\left(\ell_{\bullet}^{1}\left(\mathbf{a}, \tilde{w}_{0}\right), \ldots, \ell_{\bullet}^{k}\left(\mathbf{a}, \tilde{w}_{0}\right)\right) .
$$

Then we have

$$
\tilde{\Phi}^{-1}\left(G_{J}^{\infty}\right) \cap\left(\mathbb{A}_{\text {def }} \times\left\{\tilde{w}_{0}\right\}\right)=\tilde{\varphi}_{\tilde{w}_{0}}^{-1}\left(V_{1, J}^{\infty}\right) \cap \mathbb{A}_{\text {def }}=\left(\rho_{y} \circ \tilde{\varphi}_{\tilde{w}_{0}}\right)^{-1}\left(V_{2, J}^{\infty}\right) \cap \mathbb{A}_{\text {def }} .
$$

Recall that we have $\mathbb{I}_{J}=\mathbb{I}_{y}$. Therefore

$$
\begin{aligned}
\operatorname{dim}\left(\tilde{\Phi}^{-1}\left(G_{J}^{\infty}\right) \cap\left(\mathbb{A}_{\operatorname{def}} \times\left\{\tilde{w}_{0}\right\}\right)\right) & \leqslant \operatorname{dim}\left(\left(\rho_{y} \circ \tilde{\varphi}_{\tilde{w}_{0}}\right)^{-1}\left(V_{2, J}^{\infty}\right)\right) \\
& \leqslant \operatorname{dim} V_{2, J}^{\infty}+\operatorname{dim} \operatorname{ker}\left(\rho_{y} \circ \tilde{\varphi}_{\tilde{w}_{0}}\right) \\
& \leqslant \operatorname{dim} V_{2, J}^{\infty}+\operatorname{dim} \mathbb{A}-\operatorname{rank}\left(\rho_{y} \circ \tilde{\varphi}_{\tilde{w}_{0}}\right) \\
& =\operatorname{dim} V_{2, J}^{\infty}+\operatorname{dim} \mathbb{A}-k \# \mathbb{I}_{y} .
\end{aligned}
$$

Since

$$
\begin{aligned}
\operatorname{dim}\left(V_{2, J}^{\infty}\right) & =\operatorname{dim}\left(\mathbb{C}^{\mathbb{I}_{J}}\right)^{k}-\operatorname{codim}\left(V_{2, J}^{\infty},\left(\mathbb{C}^{\mathbb{I}_{y}}\right)^{k}\right) \\
& =k \# \mathbb{I}_{J}-\operatorname{codim}\left(V_{2, J}^{\infty},\left(\mathbb{C}^{\mathbb{I}_{y}}\right)^{k}\right) \\
& =k \# \mathbb{I}_{y}-\operatorname{codim}\left(V_{2, J}^{\infty},\left(\mathbb{C}^{\mathbb{I}_{y}}\right)^{k}\right)
\end{aligned}
$$

by putting the above inequalities together, one obtains

$$
\operatorname{dim}\left(\tilde{\Phi}^{-1}\left(G_{J}^{\infty}\right) \cap \mathbb{A}_{\text {def }} \times\left\{\tilde{w}_{0}\right\}\right) \leqslant \operatorname{dim} \mathbb{A}-\operatorname{codim}\left(V_{2, J}^{\infty},\left(\mathbb{C}^{\mathbb{I}_{y}}\right)^{k}\right),
$$

which yields

$$
\operatorname{dim}\left(\tilde{\Phi}^{-1}\left(G_{J}^{\infty}\right) \cap \mathbb{A}_{\text {def }} \times \widetilde{\mathbb{L}}_{k, J}\right) \leqslant \operatorname{dim} \mathbb{A}-\operatorname{codim}\left(V_{2, J}^{\infty},\left(\mathbb{C}^{\mathbb{I}_{y}}\right)^{k}\right)+\operatorname{dim} \widetilde{\mathbb{L}}_{k, J} .
$$


By a result due to Benoist (see [Ben11] or [BD18, Corollary 3.2]), we have

$$
\operatorname{codim}\left(V_{2, J}^{\infty},\left(\mathbb{C}^{\mathbb{I}_{y}}\right)^{k}\right) \geqslant \delta+1
$$

Therefore, if

$$
\operatorname{dim} \widetilde{\mathbb{L}}_{k, J} \leqslant(k+1) n+1<\delta+1,
$$

$\tilde{\Phi}^{-1}\left(G_{J}^{\infty}\right)$ doesn't dominate $\mathbb{A}_{\text {def }}$ via the projection $\mathbb{A}_{\text {def }} \times \widetilde{\mathbb{L}}_{k, J} \rightarrow \mathbb{A}_{\text {def }}$, and hence there exists a non-empty Zariski open subset $\mathbb{A}_{J} \subset \mathbb{A}_{\text {def }}$ such that

$$
\tilde{\Phi}^{-1}\left(G_{J}^{\infty}\right) \cap\left(\mathbb{A}_{J} \times \widetilde{\mathbb{L}}_{k, J}\right)=\varnothing .
$$

4.5. Proof of the logarithmic Kobayashi conjecture. We are now in position to conclude the proof of our first main result. With the notation of Lemma 4.3, set

$$
\mathbb{A}_{\text {nef }}:=\bigcap_{J \subset\{0, \ldots, k\}} \mathbb{A}_{J}
$$

which is a non-empty Zariski open subset $\mathbb{A}_{\text {def }}$ by Lemma 4.3. Fix $\delta=(k+1) n+k$ so that the conditions in Lemmas 4.3 and 3.6 are fulfilled.

Theorem 4.4. Same notation as above. For any $\mathbf{a} \in \mathbb{A}_{\text {nef }}$, the line bundle

$$
\nu_{k}^{*}\left(\mathscr{O}_{\mathbb{L}_{k}}\left(\delta^{k-1} k^{\prime}\right) \otimes\left(p \circ \pi_{0, k}\right)^{*} A^{\delta^{k-1} k(\varepsilon+k \delta)-r}\right) \otimes \mathscr{O}_{\widetilde{\mathbb{L}}_{k}}\left(-\delta^{k-1} F\right)_{\uparrow \widetilde{H}_{\mathbf{a}, k}}
$$

is nef on $\widetilde{H}_{\mathbf{a}, k}$.

Proof. It suffices to show that for any irreducible curve $C \subset \widetilde{H}_{\mathbf{a}, k}$, one has

$$
C \cdot \nu_{k}^{*}\left(\mathscr{O}_{\mathbb{L}_{k}}\left(\delta^{k-1} k^{\prime}\right) \otimes\left(p \circ \pi_{0, k}\right)^{*} A^{\delta^{k-1} k(\varepsilon+k \delta)-r}\right) \otimes \mathscr{O}_{\widetilde{\mathbb{L}}_{k}}\left(-\delta^{k-1} F\right) \geqslant 0 .
$$

By (4.3.2) this is equivalent to

$$
C \cdot \widetilde{\Psi}^{*}\left(\mathscr{L}^{\delta^{k-1}} \otimes \mathscr{O}_{\mathbb{P} k}(-1)\right) \geqslant 0 .
$$

Let $J \subset\{0, \ldots, k\}$ be such that $\widetilde{H}_{\mathbf{a}, k, J}$ contains a non-empty open subset $C^{\circ}$ of $C$ (there exists a unique such $J)$. By the factorization property in Lemma 4.1, one has $\widetilde{\Psi}(C) \subset \mathscr{Y}_{J}$. Moreover, by Lemma 4.3 , we see that

$$
\widetilde{\Psi}\left(C^{\circ}\right) \cap E_{J} \subset \widetilde{\Psi}\left(C^{\circ}\right) \cap \operatorname{pr}_{J}^{-1}\left(G_{J}^{\infty}\right)=\varnothing .
$$

In particular, $\widetilde{\Psi}(C) \not \subset \operatorname{pr}_{J}^{-1}\left(G_{J}^{\infty}\right)$ and therefore it follows from Theorem 4.2 applied to $m=\delta^{k-1}$ that

$$
\widetilde{\Psi}(C) \not \subset \operatorname{Bs}\left(\mathscr{L}^{\delta^{k-1}} \otimes \mathscr{O}_{\mathbb{P}^{k}}(-1) \mathscr{Y}_{J}\right),
$$

from which (4.5.5) follows at once.

Observe that Theorem 4.4 implies the following result.

Corollary 4.5. Same notation as above. There exists $\beta, \tilde{\beta} \in \mathbb{N}$ such that for any $\alpha \geqslant 0$, and for a general hypersurface $D \in\left|A^{\varepsilon+(r+k) \delta}\right|$, denoting by $Y_{k}(D)$ the log Demailly $k$-jet tower associated to $\left(Y, D, T_{Y}(-\log D)\right)$, the stable base locus

$$
\mathbf{B}\left(\mathscr{O}_{Y_{k}(D)}\left(\beta+\alpha \delta^{k-1} k^{\prime}\right) \otimes \pi_{0, k}^{*} A^{\tilde{\beta}+\alpha\left(\delta^{k-1} k(\varepsilon+k \delta)-r\right)}\right) \subseteq Y_{k}(D)^{\operatorname{sing}} \cup \pi_{0, k}^{-1}(D) .
$$

Proof. Fix any $\mathbf{a} \in \mathbb{A}_{\text {nef }}$. Observe now that there exists $\tilde{\beta}, a_{1}, \ldots, a_{k}, q \in \mathbb{N}$ such that the line bundle

$$
\mu_{\mathbf{a}, k}^{*}\left(\mathscr{O}_{H_{\mathbf{a}, k}}\left(a_{1}, \ldots, a_{k}\right) \otimes \pi_{0, k}^{*} A^{\tilde{\beta}}\right) \otimes \mathscr{O}_{\widetilde{H}_{\mathbf{a}, k}}(-q F)
$$

is ample, where $\mu_{\mathbf{a}, k}: \widetilde{H}_{\mathbf{a}, k} \rightarrow H_{\mathbf{a}, k}$ is the blow-up of the logarithmic Wronskian ideal sheaf $\mathfrak{w}_{H_{\mathbf{a}, k}}$. By Theorem 4.4 as well as the functorial properties for the restriction of Wronskians in 
(2.3.10) and the blow-up of logarithmic Wronskian ideal sheaves in Lemma 2.8, for any $\alpha \in \mathbb{N}$ the line bundle

$$
\begin{array}{r}
\left(\nu_{k}^{*}\left(\mathscr{O}_{\mathbb{L}_{k}}\left(a_{1}, \ldots, a_{k}+\alpha \delta^{k-1} k^{\prime}\right) \otimes\left(p \circ \pi_{0, k}\right)^{*} A^{\tilde{\beta}+\alpha\left(\delta^{k-1} k(\varepsilon+k \delta)-r\right)}\right) \otimes \mathscr{O}_{\widetilde{\mathbb{L}}_{k}}\left(-\left(q+\delta^{k-1}\right) F\right)\right)_{\left\lceil\widetilde{H}_{\mathbf{a}, k}\right.}= \\
\mu_{\mathbf{a}, k}^{*}\left(\mathscr{O}_{H_{\mathbf{a}, k}}\left(a_{1}, \ldots, a_{k}+\alpha \delta^{k-1} k^{\prime}\right) \otimes L_{\mathbf{a}}^{\tilde{\beta}+\alpha\left(\delta^{k-1} k(\varepsilon+k \delta)-r\right)}\right) \otimes \mathscr{O}_{\widetilde{H}_{\mathbf{a}, k}}\left(-\left(q+\delta^{k-1}\right) F\right)
\end{array}
$$

is ample, where $L_{\mathbf{a}}:=\left(p \circ \pi_{0, k}\right)^{*} A_{\uparrow H_{\mathbf{a}, k}}$. Recall that $\widetilde{H}_{\mathbf{a}, k}$ is a fiber of the smooth family $\widetilde{\mathscr{H}_{k}^{\mathrm{s}}} \rightarrow \mathbb{A}^{\mathrm{s}}$, where $\widetilde{\mathscr{H}_{k}^{s}}$ is the functorial blow-up of the universal family of log Demailly towers of general log pairs defined in $\S 2.7$. Since ampleness is an open condition in families [Laz04, Theorem 1.2.17], then by Lemma 2.8 again we conclude that there exists an non-empty Zariski open subset $\mathbb{A}_{\mathrm{amp}} \subset \mathbb{A}^{\mathrm{s}}$ such that for any $\sigma \in \mathbb{A}_{\mathrm{amp}}$, the line bundle

$$
\begin{array}{r}
\left(\nu_{k}^{*}\left(\mathscr{O}_{\mathbb{L}_{k}}\left(a_{1}, \ldots, a_{k}+\alpha \delta^{k-1} k^{\prime}\right) \otimes\left(p \circ \pi_{0, k}\right)^{*} A^{\tilde{\beta}+\alpha\left(\delta^{k-1} k(\varepsilon+k \delta)-r\right)}\right) \otimes \mathscr{O}_{\widetilde{\mathbb{L}}_{k}}\left(-\left(q+\delta^{k-1}\right) F\right)\right)_{\left\lceil\widetilde{H}_{\sigma, k}\right.}= \\
\mu_{\sigma, k}^{*}\left(\mathscr{O}_{H_{\sigma, k}}\left(a_{1}, \ldots, a_{k}+\alpha \delta^{k-1} k^{\prime}\right) \otimes L_{\sigma}^{\tilde{\beta}+\alpha\left(\delta^{k-1} k(\varepsilon+k \delta)-r\right)}\right) \otimes \mathscr{O}_{\widetilde{H}_{\sigma, k}}\left(-\left(q+\delta^{k-1}\right) F\right)
\end{array}
$$

is ample. Here $H_{\sigma, k}$ is the $\log$ Demailly $k$-jet tower of $\left(H_{\sigma}, D_{\sigma}, T_{H_{\sigma}}\left(-\log D_{\sigma}\right)\right), L_{\sigma}:=(p \circ$ $\left.\pi_{0, k}\right)^{*} A_{\left\lceil H_{\sigma, k}\right.}$, and $\mu_{\sigma, k}: \widetilde{H}_{\sigma, k} \rightarrow H_{\sigma, k}$ denotes to be the blow-up of the $k$-th log Wronskian ideal sheaf $\mathfrak{w}_{H_{\sigma, k}}$. By our construction of $H_{\sigma}$, the $\log$ pairs $\left(H_{\sigma}, D_{\sigma}\right)$ and $\left(Y, D_{\sigma}\right)$ are isomorphic. Hence the line bundle

$$
\nu_{k}^{*}\left(\mathscr{O}_{Y_{k}\left(D_{\sigma}\right)}\left(a_{1}, \ldots, a_{k}+\alpha \delta^{k-1} k^{\prime}\right) \otimes \pi_{0, k}^{*} A^{\tilde{\beta}+\alpha\left(\delta^{k-1} k(\varepsilon+k \delta)-r\right)}\right) \otimes \mathscr{O}_{\widetilde{Y}_{k}\left(D_{\sigma}\right)}\left(-\left(q+\delta^{k-1}\right) F\right)
$$

is ample as well, where we denote by $\widetilde{Y}_{k}\left(D_{\sigma}\right)$ the blow-up along the Wronskian ideal sheaf $\mathfrak{w}_{Y_{k}\left(D_{\sigma}\right)}$. In particular, its stable base locus is empty, which implies that the stable locus

$$
\mathbf{B}\left(\mathscr{O}_{Y_{k}\left(D_{\sigma}\right)}\left(a_{1}, \ldots, a_{k}+\alpha \delta^{k-1} k^{\prime}\right) \otimes \pi_{0, k}^{*} A^{\tilde{\beta}+\alpha\left(\delta^{k-1} k(\varepsilon+k \delta)-r\right)}\right)
$$

is contained in the cosupport of the logarithmic Wronskian ideal sheaf $\mathfrak{w}_{Y_{k}\left(D_{\sigma}\right)}$, which is contained in $Y_{k}\left(D_{\sigma}\right)^{\text {sing }} \cup \pi_{0, k}^{-1}\left(D_{\sigma}\right)$ by Proposition 2.5. Now it suffices to take $\beta=a_{1}+\cdots+a_{k}$ and apply the relation (1.4.12) to conclude that the stable base locus of the line bundle

$$
\mathscr{O}_{Y_{k}\left(D_{\sigma}\right)}\left(\beta+\alpha \delta^{k-1} k^{\prime}\right) \otimes \pi_{0, k}^{*} A^{\tilde{\beta}+\alpha\left(\delta^{k-1} k(\varepsilon+k \delta)-r\right)}
$$

is also contained in $Y_{k}\left(D_{\sigma}\right)^{\text {sing }} \cup \pi_{0, k}^{-1}\left(D_{\sigma}\right)$. Recall that $\mathbb{A}^{\mathrm{s}}$ is the Zariski open subset of $\mathbb{A}^{\mathrm{u}}:=$ $H^{0}\left(Y, A^{m}\right)$ parameterizing all smooth hypersurfaces, where we recall $m:=\varepsilon+(r+k) \delta$. $\mathbb{A}_{\mathrm{amp}}$ therefore parametrizes a general hypersurface in $\left|A^{m}\right|$, whence the result.

From Corollary 4.5 the first statement of our main theorem follows at once.

Corollary 4.6. Let $Y$ be a projective manifold of dimension $n \geqslant 2$, and $A$ a very ample line bundle over $Y$. Then for any

$$
m \geqslant\left(n^{2}+3 n+1\right)^{n+3} \sim_{n \rightarrow \infty} e^{3} n^{2 n+6}
$$

if $D \in\left|A^{m}\right|$ is a general smooth hypersurface, then $Y \backslash D$ is hyperbolically embedded in $Y$.

Proof. Recall first that a result of Green [Gre77] guaranties that if $D$ is a smooth hypersurface in $Y$ such that $D$ and $Y \backslash D$ are both Brody hyperbolic, then $Y \backslash D$ is hyperbolically embedded. Moreover, under the assumption of the corollary, it was established in [Bro17, Den17], that $D$ is (Brody) hyperbolic, therefore it remains to prove that $Y \backslash D$ is Brody hyperbolic. To see this we will just give an explicit bound on the degrees $\varepsilon+(r+k) \delta$ covered by Corollary 4.5. Therefore recall that we have $k=n+1$, and take $\delta=(k+1) n+k=n^{2}+3 n+1$ and set

$$
r_{0}=\delta^{k-1}(\delta+1)^{2}=\left(n^{2}+3 n+1\right)^{n}\left(n^{2}+3 n+2\right)^{2} .
$$

By the basic inequality

$$
k(k+\delta-1+k \delta)<(\delta+1)^{2}
$$


one can show that any $m \geqslant\left(r_{0}+k\right) \delta+2 \delta$ can be written in the form

$$
m=\varepsilon+(r+k) \delta
$$

with $k \leqslant \varepsilon \leqslant k+\delta-1$, and $r>\delta^{k-1} k(\varepsilon+k \delta)$. In particular, applying Corollary 4.5 for $\alpha$ large enough and applying Theorem 1.4, we see that for general hypersurface $D \in\left|A^{m}\right|, Y \backslash D$ are Brody hyperbolic. In order to obtain an explicit bound on $m$ it then suffices to give a bound on $\left(r_{0}+k\right) \delta+2 \delta$ :

$$
\begin{aligned}
\left(r_{0}+k\right) \delta+2 \delta & =\left(\delta^{k-1}(\delta+1)^{2}+k+2\right) \delta \\
& <(n+2)^{n+3}(n+1)^{n+3} \sim_{n \rightarrow \infty} e^{3} n^{2 n+6}
\end{aligned}
$$

4.6. Application to value distribution theory. In this section, we show how Corollary 4.5 allows us to obtain a result in Nevanlinna theory. Let us recall the main definition used in Nevanlinna theory and refer the reader to the book [NW14] for a detailed presentation. Let $X$ be a projective manifold and let $A$ be an ample line bundle on $X$ endowed with a smooth hermitian metric $h$ whose curvature tensor $\sqrt{-1} \Theta_{h, A}$ satisfies $\sqrt{-1} \Theta_{h, A} \geqslant \omega$ for some Kähler form $\omega$. For any entire curve $f: \mathbb{C} \rightarrow X$, the Nevanlinna order function is defined by

$$
T_{f}(r, A):=\int_{1}^{r} \frac{d t}{t} \int_{\Delta(t)} f^{*}\left(\sqrt{-1} \Theta_{h, A}\right),
$$

where $\Delta(t)$ is the disc of radius $t$ in $\mathbb{C}$. For any simple normal crossing divisor $D$ such that $f$ is not contained in $D$, and for any $k \in \mathbb{N}^{*} \cup\{\infty\}$ one sets

$$
n_{f}^{(k)}(t, D):=\sum_{|z|<t} \min \left\{k, \operatorname{mult}_{z}\left(f^{*} D\right)\right\} \quad \text { for any } t>0,
$$

where $\operatorname{mult}_{z}\left(f^{*} D\right)$ denotes the multiplicity of $f^{*} D$ at the point $z$. For $k=\infty$ one just writes $n_{f}(t, D)$. One then defines the truncated counting function at order $k$ by

$$
N_{f}^{(k)}(r, D):=\int_{1}^{r} n_{f}^{(k)}(t, D) d t \quad r>1
$$

In the case $k=\infty$ we simply write $N_{f}(r, D)$ and call it Nevanlinna's counting function. One of the purpose of Nevanlinna theory is to compare the order function and the counting functions. If for instance $D \in|A|$ then it is known that for all $r \geqslant 0$, one has

$$
N_{f}(r, D) \leqslant T_{f}(r, A)+O(1)
$$

where $O(1)$ is some bounded function. The so called "Second Main Theorems" are inequalities in the opposite direction of the form

$$
T_{f}\left(r, K_{X}\right)+T_{f}(r, A) \leqslant N_{f}(r, D)+S_{f}(r) \quad \|,
$$

where $S_{f}(r)$ is a small term compared to $T_{f}(r, A)$, and where $\|$ means that the inequality holds outside a set of finite Lebesgue measure in $\mathbb{R}^{+}$. Those inequalities are mainly conjectural and we refer to [NW14] for a detailed account on the main known second main theorem type results. In the rest of this section we will consider the following weaker version of the Second Main Theorem, which consists in establishing inequalities of the form

$$
T_{f}(f, A) \leqslant c N_{f}(r, D)+S_{f}(r) \quad \|
$$

for some constant $c$. The theory of jet differentials provides a direct way to produce such inequalities. This relies mainly on the lemma on logarithmic derivatives and appears in several places in the literature more or less explicitly (see e.g. [Yam15, Corollary 4.9]). Here we will apply the following precise statement recently established in [HVX17, Theorem 3.1]. 
Lemma 4.7. Let $(X, D)$ be a smooth logarithmic pair, and let $A$ be an ample line bundle on $X$. For any positive integers $k, N, N^{\prime}$, for any global jet differential $P \in H^{0}\left(Y, E_{k, N}^{\mathrm{GG}} \Omega_{X}(\log D) \otimes\right.$ $\left.A^{-N^{\prime}}\right)$, and for any entire curve $f: \mathbb{C} \rightarrow X$ which is not contained in $\operatorname{Supp}(D)$, if $f^{*} P \not \equiv 0$, then there exists a constant $C$ such that

$$
T_{f}(r, A) \leqslant \frac{N}{N^{\prime}} \cdot N^{(1)}(f, D)+C\left(\log T_{f}(r, L)+\log r\right) \quad \| .
$$

Here the symbol || means that the inequality holds outside a Borel subset of $(0,+\infty)$ of finite Lebesgue measure.

Let us mention that in [HVX17] the authors only state their result in the case $X=\mathbb{P}^{n}$, but this restriction is unnecessary.

As an immediate consequence of Lemma 4.7 we obtain the following.

Corollary 4.8. Let $(X, D)$ be a smooth log pair, and let $A$ be an ample line bundle on $X$. Let $\pi_{0, k}: X_{k}(D) \rightarrow X$ be the log Demailly tower associated to the pair $(X, D)$. For any positive integers $k, N, N^{\prime}$, if the stable base locus

$$
\mathbf{B}\left(\mathscr{O}_{X_{k}(D)}(N) \otimes \pi_{0, k}^{*} A^{-N^{\prime}}\right) \subseteq X_{k}(D)^{\operatorname{sing}} \bigcup \pi_{0, k}^{-1}(D)
$$

then, for any entire curve $f: \mathbb{C} \rightarrow X$ not contained in $\operatorname{Supp}(D)$, one has

$$
T_{f}(r, A) \leqslant \frac{N}{N^{\prime}} \cdot N^{(1)}(f, D)+C\left(\log T_{f}(r, A)+\log r\right) \quad \| .
$$

It now suffices to combine this result with Corollary 4.5 to obtain a Second Main Theorem type result for general log pairs.

Corollary 4.9. Let $Y$ be a projective manifold of dimension $n \geqslant 2$, and let $A$ be a very ample line bundle over $Y$. If $D \in\left|A^{m}\right|$ is a general smooth hypersurface with

$$
m \geqslant(n+2)^{n+3}(n+1)^{n+3} \sim_{n \rightarrow \infty} e^{3} n^{2 n+6},
$$

then for any entire curve $f: \mathbb{C} \rightarrow Y$ not contained in $\operatorname{Supp}(D)$, there exists $C \in \mathbb{R}^{+}$such that

$$
T_{f}(r, A) \leqslant N^{(1)}(f, D)+C\left(\log T_{f}(r, A)+\log r\right) \quad \| .
$$

Proof. Let us take $k=n+1, \delta=(k+1) n+k=n^{2}+3 n+1$ and set

$$
r_{0}=\delta^{k-1} k^{\prime}+\delta^{k-1}(\delta+1)^{2}=\delta^{k-1}(\delta+1)\left(\delta+\frac{3}{2}\right) .
$$

By (4.5.6) one can prove that any $m \geqslant\left(r_{0}+k\right) \delta+2 \delta$ can be written in the form

$$
m=\varepsilon+(r+k) \delta
$$

with $k \leqslant \varepsilon \leqslant k+\delta-1$, and $r>\delta^{k-1} k^{\prime}+\delta^{k-1} k(\varepsilon+k \delta)$. In particular, applying Corollary 4.5 for $\alpha \gg 0$ such that $-\tilde{\beta}-\alpha\left(\delta^{k-1} k(\varepsilon+k \delta)-r\right)>0$ and applying Corollary 4.8 we see that for such $m$, general hypersurface $D \in\left|A^{m}\right|$ and for any entire curve $f: \mathbb{C} \rightarrow Y$ not contained in $\operatorname{Supp}(D)$, there exists $C \in \mathbb{R}^{+}$such that

$$
T_{f}(r, A) \leqslant \frac{\beta+\alpha \delta^{k-1} k^{\prime}}{-\tilde{\beta}-\alpha\left(\delta^{k-1} k(\varepsilon+k \delta)-r\right)} \cdot N^{(1)}(f, D)+C\left(\log T_{f}(r, A)+\log r\right) \quad \| .
$$

However,

$$
\frac{\beta+\alpha \delta^{k-1} k^{\prime}}{-\tilde{\beta}-\alpha\left(\delta^{k-1} k(\varepsilon+k \delta)-r\right)} \rightarrow_{\alpha \rightarrow \infty} \frac{\delta^{k-1} k^{\prime}}{r-\left(\delta^{k-1} k(\varepsilon+k \delta)\right.}<1 .
$$

Therefore, in order to complete the proof, it now suffices to give a bound on $\left(r_{0}+k\right) \delta+2 \delta$ :

$$
\begin{aligned}
\left(r_{0}+k\right) \delta+2 \delta & =\left(\delta^{k-1}(\delta+1)\left(\delta+\frac{3}{2}\right)+k+2\right) \delta \\
& <(n+2)^{n+3}(n+1)^{n+3} \lesssim_{n \rightarrow \infty} e^{3} n^{2 n+6} .
\end{aligned}
$$


4.7. Orbifold hyperbolicity and hyperbolicity for the cyclic cover. The orbifold introduced by Campana arises naturally in his study of the birational classification of varieties in [Cam04]. One can also generalize the definition of Kobayashi hyperbolicity and the tools of jet differentials to orbifolds, which were first studied by Rousseau in [Rou10]. We refer the readers to the very recent paper [CDR18] for the hyperbolicity and orbifold jet differentials in the orbifold category. In this last section, we will apply Corollary 4.5 to prove the orbifold hyperbolicity for general orbifolds. From [CDR18] one can easily derive the following lemma.

Lemma 4.10. Let $Y$ be an $n$-dimensional projective manifold, and let $D$ be a smooth hypersurface of $Y$. Then for the Campana orbifold $(Y, \Delta):=\left(Y,\left(1-\frac{1}{m}\right) D\right)$ where $m \in \mathbb{N}^{*}$, one has natural inclusions

$$
E_{k, N}^{\mathrm{GG}} \Omega_{Y}(\log D) \otimes \mathscr{O}_{Y}\left(-\left\lceil\frac{N}{m}\right\rceil D\right) \hookrightarrow E_{k, N}^{\mathrm{GG}} \Omega_{Y, \Delta} \hookrightarrow E_{k, N}^{\mathrm{GG}} \Omega_{Y}(\log D)
$$

where $E_{k, N}^{\mathrm{GG}} \Omega_{Y, \Delta}$ is the orbifold jet differential of degree $k$ and weight $N$ defined in [CDR18].

Proof. Take any open subset of $U \subset Y$ with local coordinates $\left(z_{1}, \ldots, z_{n}\right)$ such that $D \cap U=$ $\left(z_{1}=0\right)$. By Lemma 1.1, for any $j \in \mathbb{N}, \frac{d^{j} z_{1}}{z_{1}}$ is a logarithmic jet differential and moreover, $E_{k, N}^{\mathrm{GG}} \Omega_{Y}(\log D)$ is the locally free sheaf generated in local coordinates by elements

$$
\left\{\frac{1}{z_{1}^{\alpha_{1}^{1}+\cdots+\alpha_{k}^{1}}}\left(d^{1} z\right)^{\alpha_{1}}\left(d^{2} z\right)^{\alpha_{2}} \cdots\left(d^{k} z\right)^{\alpha_{k}}\right\}_{\left|\alpha_{1}\right|+2\left|\alpha_{2}\right|+\cdots+k\left|\alpha_{k}\right|=N}
$$

where $\alpha_{j}=\left(\alpha_{j}^{1}, \ldots, \alpha_{j}^{n}\right) \in \mathbb{N}^{n}$. By [CDR18, 2.3$] E_{k, N}^{\mathrm{GG}} \Omega_{Y, \Delta}$ is the locally free subsheaf of $E_{k, N} \Omega_{Y}(\log D)$ generated in local coordinates by elements

$$
\left\{z_{1}^{\left\lceil\frac{\alpha_{1}^{1} \min (1, m)+\cdots+k \alpha_{k}^{1} \min (k, m)}{m}\right\rceil} \cdot \frac{1}{z_{1}^{\alpha_{1}^{1}+\cdots+\alpha_{k}^{1}}}\left(d^{1} z\right)^{\alpha_{1}}\left(d^{2} z\right)^{\alpha_{2}} \cdots\left(d^{k} z\right)^{\alpha_{k}}\right\}_{\left|\alpha_{1}\right|+2\left|\alpha_{2}\right|+\cdots+k\left|\alpha_{k}\right|=N}
$$

The lemma then follows immediately from the obvious inequality

$$
\left\lceil\frac{\alpha_{1}^{1} \min (1, m)+\cdots+k \alpha_{k}^{1} \min (k, m)}{m}\right\rceil \leqslant\left\lceil\frac{N}{m}\right\rceil .
$$

Now let us combine Lemma 4.10 with Corollary 4.5 to prove the orbifold hyperbolicity.

Corollary 4.11. Let $Y$ be a projective manifold of dimension $n \geqslant 2$, and $A$ a very ample line bundle over $Y$. Then for any

$$
m \geqslant(n+2)^{n+3}(n+1)^{n+3} \sim_{n \rightarrow \infty} e^{3} n^{2 n+6},
$$

if $D \in\left|A^{m}\right|$ is a general smooth hypersurface,

(i) the orbifold $(Y, \Delta):=\left(Y,\left(1-\frac{1}{m}\right) D\right)$ is orbifold hyperbolic.

(ii) For the cyclic cover $\pi: X \rightarrow Y$ obtained by taking the $m$-th root along $D, X$ is Kobayashi hyperbolic.

Proof. As in the proof of Corollary 4.9, we take $k=n+1, \delta=(k+1) n+k=n^{2}+3 n+1$ and

$$
r_{0}=\delta^{k-1}(\delta+1)\left(\delta+\frac{3}{2}\right) \text {. }
$$

Then by the computations therein and Corollary 4.5, for any general smooth hypersurface $D \in\left|A^{m}\right|$ with

$$
m \geqslant(n+2)^{n+3}(n+1)^{n+3},
$$

we can take $\alpha \gg 0$ so that

$$
N:=\beta+\alpha \delta^{k-1} k^{\prime}<N^{\prime}:=-\tilde{\beta}-\alpha\left(\delta^{k-1} k(\varepsilon+k \delta)-r\right)
$$

with

$$
\mathbf{B}\left(\mathscr{O}_{Y_{k}(D)}(N) \otimes \pi_{0, k}^{*} A^{-N^{\prime}}\right) \subseteq Y_{k}(D)^{\text {sing }} \cup \pi_{0, k}^{-1}(D)
$$


By (1.4.13), for any $\ell \gg 0$ one has sufficiently many $\log$ jet differentials in $E_{k, \ell N} \Omega_{Y}(\log D) \otimes$ $\mathscr{O}_{Y}\left(-\ell N^{\prime} A\right)$ in the sense that, for any germ of curve $\gamma:(\mathbb{C}, 0) \rightarrow(Y \backslash D, y)$ whose $k$-jet $j_{k} \gamma(0) \neq 0$, there always exists a logarithmic jet differential

$$
P \in H^{0}\left(Y, E_{k, \ell N} \Omega_{Y}(\log D) \otimes \mathscr{O}_{Y}\left(-\ell N^{\prime} A\right)\right)
$$

with $P\left(j_{k} \gamma\right)(0) \neq 0$. By the inclusive relation in Lemma 4.10, one also has sufficiently many orbifold jet differentials in

$E_{k, \ell N} \Omega_{Y, \Delta}(\log D) \otimes \mathscr{O}_{Y}\left(-\ell N^{\prime} A+\left\lceil\frac{\ell N}{m}\right\rceil D\right)=E_{k, \ell N} \Omega_{Y, \Delta}(\log D) \otimes \mathscr{O}_{Y}\left(\left(-\ell N^{\prime}+m\left\lceil\frac{\ell N}{m}\right\rceil\right) A\right)$.

Take $\ell$ divisible enough (i.e. $m \mid \ell$ ) and one thus has

$$
\ell N^{\prime}-m\left\lceil\frac{\ell N}{m}\right\rceil>0
$$

which implies the orbifold hyperbolicity of $(Y, \Delta)$ by the fundamental vanishing theorem in the orbifold setting (cf. [CDR18, Corollary 3.11]). Hence the first claim is proved.

To prove the second statement, since $X$ is compact, it is equivalent to show that $X$ is also Brody hyperbolic. To prove this, we assume that there exists an entire curve $f: \mathbb{C} \rightarrow X$ on $X$, and the contradiction is derived immediately by observing that $\pi \circ f: \mathbb{C} \rightarrow Y$ is an orbifold entire curve with respect to the orbifold $(Y, \Delta)$, whereas $(Y, \Delta)$ is orbifold hyperbolic by the first claim. This proves the second claim.

Let us mention that in Corollaries 4.6, 4.9 and 4.11 we made an approximation in order to give readable bound. In all cases, as is clear from the proof, we could obtain a slightly better bound. The fact that the same bound appears in Corollary 4.6 and Corollary 4.9 is due to this approximation. In fact, our method would provide a slightly better bound in Corollary 4.6 than that in Corollaries 4.9 and 4.11 .

\section{NOTATIONS}

$f:(\mathbb{C}, 0) \rightarrow X \quad$ Germ of holomorphic curve

$J_{k} X \rightarrow X$

$j_{k} f \in J_{k} X$

$J_{k}(X, \log D)$

$E_{k, m}^{\mathrm{GG}} \Omega_{X}, E_{k, m}^{\mathrm{GG}} \Omega_{X}(\log D)$

$(X, V)$

$X_{k}$

$(X, D, V)$

$E_{k, m} \Omega_{X}, E_{k, m} \Omega_{X}(\log D)$ $X_{k}(D)$

$\pi_{0, k}: X_{k}(D) \rightarrow X$

$J^{k} L$

$j_{L}^{k} s \in H^{0}\left(X, J^{k} L\right)$

$W_{L}(\bullet)$

$W_{D}(\bullet)$

$\omega_{D}(\bullet)$

$\nabla_{\mathfrak{U}}^{j}(\bullet)$

$\omega_{D}^{\prime}(\bullet)$

$\mathfrak{w}_{X_{k}(D)}$

$\mathbb{L}$

$W_{\mathbb{L}, Y}(\bullet)$
Fiber bundle of $k$-jets of germs of holomorphic curves in $X$ $k$-jet of germ of curve $f$ in $J_{k} X$

Fiber bundle of logarithmic $k$-jets of germs of holomorphic curves in $X$

Green-Griffiths bundle of (logarithmic) jet differentials of order $k$ and weight $m$

Directed manifolds

Demailly-Semple $k$-jet tower of the directed manifold $(X, V)$

Logarithmic directed manifold

Invariant (logarithmic) jet bundle of order $k$ and weight $m$

logarithmic Demailly(-Semple) $k$-jet tower associated to logarithmic

directed manifold $\left(X, D, T_{X}(-\log D)\right)$

The natural projection map

Jet bundle of a line bundle $L$ (1.5.14)

$k$-jet of the holomorphic section $s \in H^{0}(X, L)$

Wronskian (2.1.1) associated to the line bundle $L$

Logarithmic Wronskian associated to log pair $(X, D)(2.3 .6)$

$\left(\pi_{0, k}\right)_{*} \omega_{D}(\bullet)=W_{D}(\bullet)(2.3 .8)$

Higher order logarithmic connection in the trivialization tower $\mathfrak{U}$ (2.4.11)

Defined in (2.4.12) or (2.4.18)

$k$-th logarithmic Wronskian ideal sheaf of the log manifold $(X, D)$

The total space of the line bundle $A^{\otimes m}$ on $Y$

Logarithmic Wronskian associated to the log pair $(\mathbb{L}, Y)$ 


$$
\begin{aligned}
& \mathbb{L}_{k} \\
& \omega_{\log }(\bullet) \\
& \mathfrak{w}_{k, \mathbb{L}, Y}, \mathfrak{w}_{k, \mathbb{L}, Y}^{\prime} \\
& \nu_{k}: \widetilde{\mathbb{L}}_{k} \rightarrow \mathbb{L}_{k} \\
& \left(H_{\sigma}, D_{\sigma}\right) \\
& H_{\sigma, k} \\
& \mu_{\sigma, k}: \widetilde{H}_{\sigma, k} \rightarrow H_{\sigma, k} \\
& \mathbf{a} \in \mathbb{A} \\
& (\mathscr{H}, \mathscr{D}) \rightarrow \mathbb{A}_{\mathrm{sm}} \\
& \mathscr{H}_{k} \\
& \omega_{\log , I_{1}, \ldots, I_{k}}(\bullet) \\
& E_{k, N}^{\mathrm{GG}} \Omega_{Y, \Delta}
\end{aligned}
$$

Log Demailly $k$-jet tower of $\log$ directed manifold $\left(\mathbb{L}, Y, T_{\mathbb{L}}(-\log Y)\right)$ $\left(\pi_{0, k}\right)_{*} \omega_{\log }(\bullet)=W_{\mathbb{L}, Y}(\bullet)$

Ideal sheaves of $\mathbb{L}_{k}$ in (2.6.20)

The blow-up of $\mathfrak{w}_{k, \mathbb{L}, Y}$ and $\mathfrak{w}_{k, \mathbb{L}, Y}^{\prime}$

The sub-log manifold of the log pair $(\mathbb{L}, Y)$ induced by $\sigma \in H^{0}\left(Y, A^{m}\right)$ Log Demailly tower of $\log$ directed manifold $\left(H_{\sigma}, D_{\sigma}, T_{H_{\sigma}}\left(-\log D_{\sigma}\right)\right)$ The blow-up of $\mathfrak{w}_{H_{\sigma, k}}$

Family of hypersurfaces in $Y$ parametrized by certain Fermat-type hypersurfaces defined in $\S 3.1$

Smooth family of sub-log pairs of $(\mathbb{L}, Y)$ induced by Fermat-type hypersurfaces

Log Demailly $k$-jet tower of log directed manifold $\left(\mathscr{H}, \mathscr{D}, T_{\mathscr{H} / \mathbb{A}_{\mathrm{sm}}}(-\log \mathscr{D})\right)$ Modified logarithmic Wronskians (3.2.2)

Orbifold jet differentials of order $k$ and weight $N$ associated to Campana orbifold $(Y, \Delta)$.

\section{REFERENCES}

[BD17] Damian Brotbek and Ya Deng, On the positivity of the logarithmic cotangent bundle, arXiv:1712.09887, to appear in Ann. Inst. Fourier (en l'honneur du professeur Jean-Pierre Demailly) (2017).

[BD18] Damian Brotbek and Lionel Darondeau, Complete intersection varieties with ample cotangent bundles, Invent. Math. 212 (2018), no. 3, 913-940. MR 3802300

[Ben11] Olivier Benoist, Le théorème deBertini en famille, Bulletin de la Société Mathématique de France 139 (2011), no. 4, 555-569.

[Bro78] Robert Brody, Compact manifolds and hyperbolicity, Trans. Amer. Math. Soc. 235 (1978), 213-219. MR 0470252

[Bro17] Damian Brotbek, On the hyperbolicity of general hypersurfaces, Publ. Math. Inst. Hautes Études Sci. 126 (2017), 1-34. MR 3735863

[Cam04] Frédéric Campana, Orbifolds, special varieties and classification theory, Ann. Inst. Fourier (Grenoble) 54 (2004), no. 3, 499-630. MR 2097416

[CDR18] Frédéric Campana, Lionel Darondeau, and Erwan Rousseau, Orbifold hyperbolicity, arXiv e-prints (2018), arXiv:1803.10716.

[Dar16a] Lionel Darondeau, Fiber integration on the Demailly tower, Ann. Inst. Fourier (Grenoble) 66 (2016), no. 1, 29-54. MR 3477869

[Dar16b] _ On the logarithmic Green-Griffiths conjecture, Int. Math. Res. Not. IMRN (2016), no. 6, 1871-1923. MR 3509943

[Dar16c] _ Slanted vector fields for jet spaces, Math. Z. 282 (2016), no. 1-2, 547-575. MR 3448395

[Dem97] Jean-Pierre Demailly, Algebraic criteria for Kobayashi hyperbolic projective varieties and jet differentials, Algebraic geometry - SantaCruz 1995, Proc. Sympos. Pure Math., vol. 62, Amer. Math. Soc., Providence, RI, 1997, pp. 285-360. MR 1492539 (99b:32037)

[Dem18] - Recent results on the Kobayashi and Green-Griffiths-Lang conjectures, arXiv e-prints (2018), arXiv:1801.04765.

[Den17] Ya Deng, On the Diverio-Trapani Conjecture, arXiv:1703.07560, to appear in Ann. Sci. Éc. Norm. Supér. (2017).

[Div09] Simone Diverio, Existence of global invariant jet differentials on projective hypersurfaces of high degree, Math. Ann. 344 (2009), no. 2, 293-315. MR 2495771 (2010c:32047)

[DL01] Gerd-Eberhard Dethloff and Steven Shin-Yi Lu, Logarithmic jet bundles and applications, Osaka J. Math. 38 (2001), no. 1, 185-237. MR 1824906

[DMR10] Simone Diverio, Joël Merker, and Erwan Rousseau, Effective algebraic degeneracy, Invent. Math. 180 (2010), no. 1, 161-223. MR 2593279

[EG03] Jawher El Goul, Logarithmic jets and hyperbolicity, Osaka J. Math. 40 (2003), no. 2, 469-491. MR 1988702

[Fuj72] Hirotaka Fujimoto, On holomorphic maps into a taut complex space, Nagoya Math. J. 46 (1972), 49-61. MR 0310274

[Gre77] Mark L. Green, The hyperbolicity of the complement of $2 n+1$ hyperplanes in general position in $\mathbb{P}_{n}$ and related results, Proc. Amer. Math. Soc. 66 (1977), no. 1, 109-113. MR 0457790 
[Gro66] A. Grothendieck, Éléments de Géométrie Algébrique. IV. étude locale des schémas et des morphismes de schémas.III, Inst. Hautes Études Sci. Publ. Math. (1966), no. 28, 255. MR 0217086

[Har77] Robin Hartshorne, Algebraic geometry, Springer-Verlag, New York-Heidelberg, 1977, Graduate Texts in Mathematics, No. 52. MR 0463157

[HVX17] Dinh Tuan Huynh, Duc-Viet Vu, and Song-Yan Xie, Entire holomorphic curves into projective spaces intersecting a generic hypersurface of high degree, arXiv:1704.03358 [math.AG] (2017).

[Kob70] Shoshichi Kobayashi, Hyperbolic manifolds and holomorphic mappings, Pure and Applied Mathematics, vol. 2, Marcel Dekker, Inc., New York, 1970. MR 0277770

[Kob98] _ Hyperbolic complex spaces, Grundlehren der Mathematischen Wissenschaften [Fundamental Principles of Mathematical Sciences], vol. 318, Springer-Verlag, Berlin, 1998. MR 1635983

[Laz04] Robert Lazarsfeld, Positivity in algebraic geometry.I, Ergebnisse der Mathematik und ihrer Grenzgebiete. 3. Folge. A Series of Modern Surveys in Mathematics [Results in Mathematics and Related Areas. 3rd Series. A Series of Modern Surveys in Mathematics], vol. 48, Springer-Verlag, Berlin, 2004, Classical setting: line bundles and linear series.

[LW12] Steven S. Y. Lu and Jörg Winkelmann, Quasiprojective varieties admittingZariski dense entire holomorphic curves, Forum Math. 24 (2012), no. 2, 399-418. MR 2900013

[Nak00] Michael Nakamaye, Stable base loci of linear series, Mathematische Annalen 318 (2000), no. 4, 837847.

[Nog86] Junjiro Noguchi, Logarithmic jet spaces and extensions of deFranchis' theorem, Contributions to several complex variables, Aspects Math., E9, Friedr. Vieweg, Braunschweig, 1986, pp. 227-249. MR 859200

[NW14] Junjiro Noguchi and Jörg Winkelmann, Nevanlinna theory in several complex variables and Diophantine approximation, Grundlehren der Mathematischen Wissenschaften [Fundamental Principles of Mathematical Sciences], vol. 350, Springer, Tokyo, 2014. MR 3156076

[NWY07] Junjiro Noguchi, Jörg Winkelmann, and Katsutoshi Yamanoi, Degeneracy of holomorphic curves into algebraic varieties, J. Math. Pures Appl. (9) 88 (2007), no. 3, 293-306. MR 2355461

[NWY08] - The second main theorem for holomorphic curves into semi-abelian varieties.II, Forum Math. 20 (2008), no. 3, 469-503. MR 2418202

[NWY13] _ Degeneracy of holomorphic curves into algebraic varieties II, Vietnam J. Math. 41 (2013), no. 4, 519-525. MR 3142409

[Rou03] Erwan Rousseau, Hyperbolicité du complémentaire d'une courbe dans $\mathbb{P}^{2}$ : le cas de deux composantes, C. R. Math. Acad. Sci. Paris 336 (2003), no. 8, 635-640. MR 1988123

[Rou09] _ Logarithmic vector fields and hyperbolicity, Nagoya Math. J. 195 (2009), 21-40. MR 2552951

[Rou10] _ Hyperbolicity of geometric orbifolds, Trans. Amer. Math. Soc. 362 (2010), no. 7, 3799-3826. MR 2601610

[RR13] Xavier Roulleau and Erwan Rousseau, On the hyperbolicity of surfaces of general type with small $c_{1}^{2}$, J. Lond. Math. Soc. (2) 87 (2013), no. 2, 453-477. MR 3046280

[RY18] Eric Riedl and David Yang, Applications of a grassmannian technique in hypersurfaces, arXiv e-prints (2018), arXiv:1806.02364.

[Siu04] Yum-Tong Siu, Hyperbolicity in complex geometry, The legacy of Niels Henrik Abel, Springer, Berlin, 2004, pp. 543-566. MR 2077584

[Siu15] Hyperbolicity of generic high-degree hypersurfaces in complex projective space, Invent. Math. 202 (2015), no. 3, 1069-1166. MR 3425387

[SY96] Yum-Tong Siu and Sai-kee Yeung, Hyperbolicity of the complement of a generic smooth curve of high degree in the complex projective plane, Invent. Math. 124 (1996), no. 1-3, 573-618. MR 1369429

[Voi96] Claire Voisin, On a conjecture of Clemens on rational curves on hypersurfaces, J. Differential Geom. 44 (1996), no. 1, 200-213. MR 1420353

[Voi98] _ A correction: "On a conjecture of Clemens on rational curves on hypersurfaces", J. Differential Geom. 49 (1998), no. 3, 601-611. MR 1669712

[Xie18] Song-Yan Xie, On the ampleness of the cotangent bundles of complete intersections, Invent. Math. 212 (2018), no. 3, 941-996. MR 3802301

[Yam15] Katsutoshi Yamanoi, Kobayashi hyperbolicity and higher-dimensional Nevanlinna theory, Geometry and analysis on manifolds, Progr. Math., vol. 308, Birkhäuser/Springer, Cham, 2015, pp. 209-273. MR 3331401 
Université de Strasbourg, Institut de Recherche Mathématique Avancée, 7 Rue René-Descartes, 67084 Strasbourg, France

Current address: Centre de mathématiques Laurent Schwartz, École polytechnique, 91128 Palaiseau CEDEX, France

E-mail address: damian.brotbek@polytechnique.edu

URL: https://sites.google.com/site/damianbrotbek/home

Université de Strasbourg, Institut de Recherche Mathématique Avancée, 7 Rue René-Descartes, 67084 Strasbourg, France

Current address: Department of Mathematical Sciences, Chalmers University of Technology and the University of Gothenburg, Sweden

E-mail address: yade@chalmers.se dengya.math@gmail.com

$U R L: \quad$ https://sites.google.com/site/dengyamath 